ORNL/TM-13298

\title{
RECEIVED
}

NOV 051996

OAK RIDGE

NATIONAL

LABORATORY

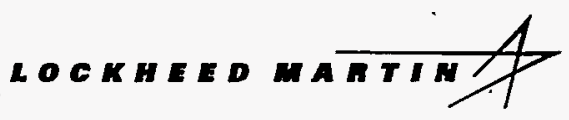

\section{Statistical Analysis of Radiochemical Measurements of TRU Radionuclides in REDC Waste}

\author{
J. Beauchamp \\ D. Downing \\ J. Chapman \\ V. Fedorov \\ L. Nguyen \\ C. Parks \\ F. Schultz \\ L. Yong
}


This report has been reproduced directly from the best available copy.

Available to DOE and DOE contractors from the Office of Scientific and Technical Information, P.O.Box 62, Oak Ridge, TN 37831; prices available from (423) 576-8401, FTS 626-8401.

Available to the public from the National Technical Information Service, U.S. Department of Commerce, 5285 Port Royal Rd., Springfield, VA 22161

This report was prepared a an account of work sponsored by an agency of the United States Government. Neither the United States Government nor any agency thereof, nor any of their employees, makes any warranty, express or implied, or assumes any legal liability or responsibility for the accuracy, completeness, or usefulness of any information, apparatus, producl, or process disclosed, or represents that its use would not infringe privately owned rights. Reference herein to any specific commercial product, process, or service by trade name, trademark, manufacturer, or olherwise, does not necessarily constitute or imply its endorsement, recommendation, or favoring by the United States Government or any agency thereof. The view and opinions of authors expressed herein do not necessarily state or reflect those of the UnitedStatesGovernment or any agency thereof. 


\title{
STATISTICAL ANALYSIS OF RADIOCHEMICAL MEASUREMENTS OF TRU RADIONUCLIDES IN REDC WASTE
}

\author{
J.Beauchamp, ${ }^{1}$ D. Downing, ${ }^{1}$ J. Chapman, ${ }^{2}$ \\ V. Fedorov, ${ }^{1}$ L. Nguyen, ${ }^{2}$ C. Parks, ${ }^{3}$ \\ F. Schultz, ${ }^{2}$ L. Yong ${ }^{2}$ \\ ${ }^{1}$ Computer Science and Mathematics Division \\ ${ }^{2}$ Waste Management and Remedial Action Division \\ ${ }^{3}$ Chemical and Analytical Sciences Division \\ Oak Ridge National Laboratory \\ P. O. Box 2008 \\ Oak Ridge, Tennessee 37831
}

Date Published: October 1996

Research sponsored by the U.S. Department of Energy TRU

Waste Program of the Office of Waste Management.

Prepared by the

Oak Ridge National Laboratory

Oak Ridge, Tennessee 37831

managed by

Lockheed Martin Energy Research Corp.

for the

U.S. DEPARTMENT OF ENERGY

under Contract No. DE-AC05-960R22464

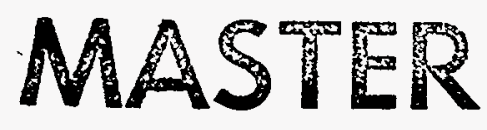

$H H$

DISTRIBUTION OF THIS DOCUMENT IS UNLRMTED 


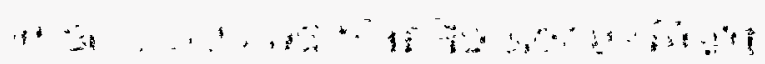




\section{DISCLAIMER}

Portions of this document may be illegible in electronic image products. Images are produced from the best available original document. 


\section{Contents}

\section{Executive Summary}

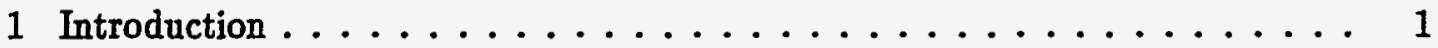

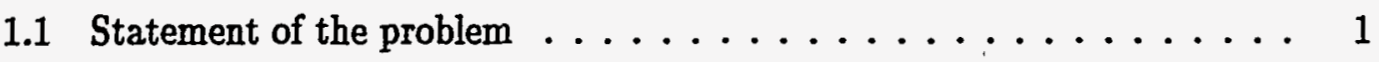

1.2 Material exposition . . . . . . . . . . . . . . . . . 3

2 Generating Processes and Main TRU. Components. . . . . . . . . . . 3

3 Sampling and Analytical Methods ................. 5

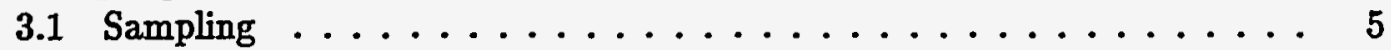

3.2 Analytical methods ......................... 6

4 Exploratory Analysis . . . . . . . . . . . . . . . . 8

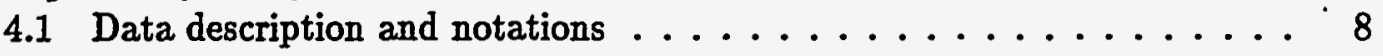

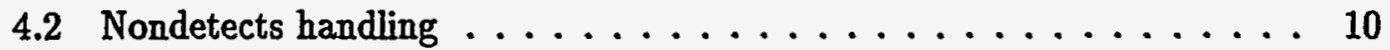

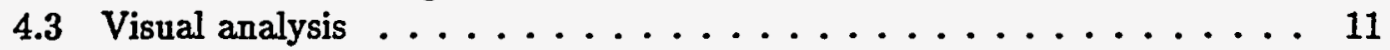

5 Estimation of TRU Isotopic Ratio ................... 13

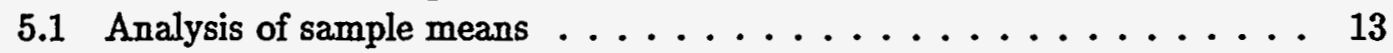

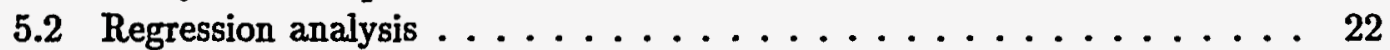

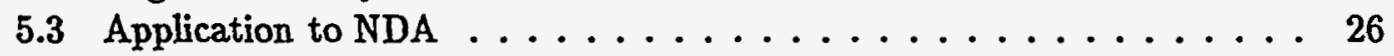

5.4 Comments on proportion TRU trend .............. 27

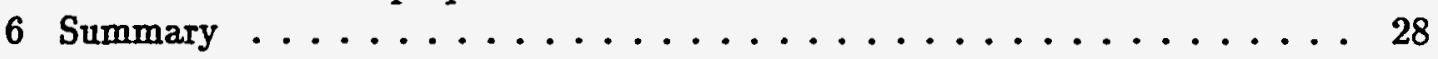

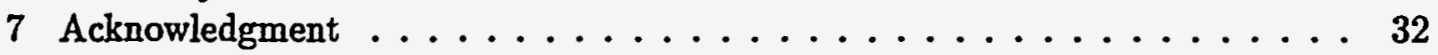

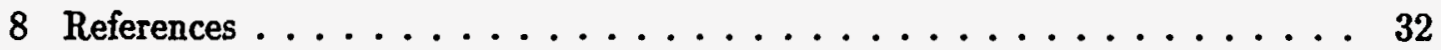

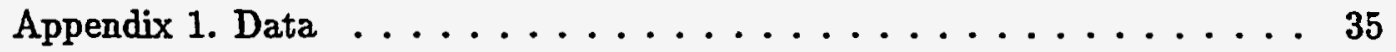

Appendix 2. Linear Regression for Poisson-Distributed Data with Special

Reference to Spectrometry . . . . . . . . . . . . . . . . . . 51

Appendix 3. Models and Analysis of Sampling Experiments . . . . . . . . 54

\section{DISCLAIMER}

This report was prepared as an account of work sponsored by an agency of the United States Government. Neither the United States Government nor any agency thereof, nor any of their employees, makes any warranty, express or implied, or assumes any legal liability or responsibility for the accuracy, completeness, or usefulness of any information, apparatus, product, or process disclosed, or represents that its use would not infringe privately owned rights. Reference herein to any specific commercial product, process, or service by trade name, trademark, manufacturer, or otherwise does not necessarily constitute or imply its endorsement, recommendation, or favoring by the United States Government or any agency thereof. The views and opinions of authors expressed herein do not necessarily state or reflect those of the United States Government or any agency thereof. 


\section{List of Tables}

1 Potential Radionuclides in REDC Solid Wastes .......... 4

2 Waste Composition ................... 5

3 (a) Estimation of TRU Proportion from First Sample Set No Observations 0 mitted . . . . . . . . . . . . . . 16

(b) Estimation of TRU Proportion from First Sample Set Two Outlier Observations Omitted .................. 17

4 (a) Estimation of TRU Proportion from Second Sample Set No Observations 0 mitted $\ldots \ldots \ldots \ldots \ldots \ldots \ldots \ldots$

(b) Estimation of TRU Proportion from Second Sample Set Two Outlier Observations from Leaching Samples Omitted . . . . . . . . . . 19

5 (a) Estimation of TRU Proportion from Both Sample Sets No Observations Omitted ..................... 20

(b) Estimation of TRU Proportion from Both Sample Sets Combined Four Outlier Observations Omitted . . . . . . . . . . . . . 21

6 Parameter Estimates from Regression of n (Proportion TRU) on $n$ (Gross Alpha) . . . . . . . . . . . . . . . 24 


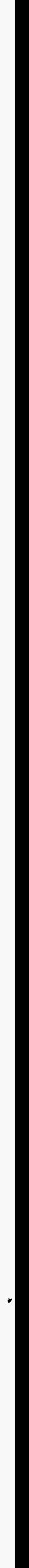




\title{
STATISTICAL ANALYSIS OF RADIOCHEMICAL MEASUREMENTS OF TRU RADIONUCLIDES IN REDC WASTE
}

\author{
J.Beauchamp, ${ }^{1}$ D. Downing, ${ }^{1}$ J. Chapman, ${ }^{2}$ \\ V: Fedorov, ${ }^{1}$ L. Nguyen,${ }^{2}$ C. Parks, ${ }^{3}$ \\ F. Schultz, ${ }^{2}$ L. Yong ${ }^{2}$
}

\begin{abstract}
This report summarizes the results of the study on the isotopic ratios of transuranium elements in waste from the Radiochemical Engineering Development Center actinide-processing streams. The knowledge of the isotopic ratios when combined with the results of various nondestructive assays, and in particular with the results of Active-Passive Neutron Examination Assay and Gamma Active Segmented Passive Assay, may lead to the significant increase in precision of the determination of TRU elements contained in ORNL generated waste streams.
\end{abstract}

\section{Executive Summary}

Radiochemistry measurements of contaminated-surface smears collected from within the REDC process and waste storage hot cells were statistically analyzed to estimate the proportion of gross alpha activity contributed by the transuranic radioisotopes $\mathrm{Cm} 246$, Pu238, Pu239, Pu240, Am241, and Am243'1 . Unlike weapons-grade plutonium, waste generated from the REDC is composed largely of non-TRU waste isotopes Cm244 and Cf252; hence the classification as TRU-waste by alpha-activity concentration alone, is difficult and requires a thorough evaluation of radiochemical results using statistical methods that address the unique aspects of the problem. This work was performed to analyze isotopic ratios of TRU radionuclides to gross alpha activity; test whether the ratios are constant over different types of debris waste; assist in the calibration of

\footnotetext{
${ }^{1} \mathrm{TRU}$ waste is, without regard to source or form, waste that is contaminated with alpha-emitting transuranium radionuclides with half-lives greater than 20 years and concentrations greater than $100 \mathrm{nCi} / \mathrm{g}$ at the time of assay [DOE Order $5820.2 \mathrm{a}]$.
} 
nondestructive assay instruments; and establish a basis for ascertaining whether the concentration of TRU radionuclides is less than the $100 \mathrm{nCi} / \mathrm{g}$ limit for classification as low-level waste.

Two sets of smears were collected from the hot cells. The majority of the first set of smears was collected in January 1996 near the end of target Campaign 69. Thirtyfour smears were collected: 26 from cell $9 ; 1$ each from cells $1,2,3,4,7$, and 8; and 2 from cell 6 . Waste material type (or component) was grouped into five classes: glass, metal, polyethylene, cell surface, and wipes. Smears were taken from each of these classes to test whether the proportion TRU was dependent on material type. The smears were analyzed by gross alpha counting in a gas flow proportional counter, alpha spectrometry, gamma-ray spectrometry, and gross neutron counting. Once the radiochemical results were available from the first set, it was realized that the plutonium sensitivity was insufficient to meet the needs of our analysis. It was concluded that a plutonium separation was required to improve the sensitivity by at least an order of magnitude from the set 1 results. Hence, a second set of smears was collected in May 1996, after Campaign 70 had begun. Twenty-six smears were collected this time, all from within cell 9 , the primary cell from which waste is generated. Waste types from this set included glass, metal, and polyethylene. Having learned from our experience of the first sample set, we decided to collect a few duplicate samples from 2 separate items, a glass bottle and a piece of metal. This allowed an evaluation of the sample-to-sample variability. Additionally, a metal tool was smeared and then leached to compare a smear result with a leach result. A smear, followed by a leach, was also performed for three glass bottles. Plutonium was removed from an aliquot of leachate by solvent extraction and then analyzed by gross alpha counting and alpha spectrometry; thereby, improving the sensitivity to plutonium. In set 2 plutonium was detected in all 26 smears, versus only 9 of 34 in set 1 . In summary, the samples collected in set 1 were from all of the cells-but mostly cell 9-with no duplicate smears taken. In contrast, set 2 was collected exclusively from cell 9; two subsets of duplicates were taken; and in two cases, smears were followed by an acid leach procedure, thus providing in two cases total radioactivity results per item. 
Parametric and nonparametric statistical methods were performed on the data, which included a total of 60 smears and 2 leach results. Parametric methods require assumptions about the analytical form of probability distributions to be made and when possible verified on the statistical distribution of the data. Nonparametric methods use very mild assumptions like stochastic independence. The variable of interest was defined as the proportion TRU, the ratio of the radioactivity sum of measured TRU radionuclides $(\mathrm{Bq})$ to gross alpha activity $(\mathrm{Bq})$. For the parametric methods, normal and lognormal distributions were assumed for the distribution of the observed variable. However, no normality assumption was required for the nonparametric methods. Using parametric and nonparametric techniques, estimates of the mean and corresponding standard errors were computed for the variable, proportion TRU. Because all of the TRU radionuclides of interest were not detected in every sample, several data censoring techniques were used. Lower and upper bounds on the proportion TRU were obtained by making assumptions (low and high) about the contributing isotopes that were not detected in a smear. We defined a nondetect observation, i.e. a censored data point, as one in which at least one of the six TRU isotopes was not detected by radiochemical analysis. For a lower bound, the radioactivities of isotopes that were not detected were set to zero; for an upper bound, the radioactivity was set to $1 \%$ of the gross alpha activity, except for plutonium. Approximately $50 \%$ cases of plutonium isotopes were not detected in set 1 (recall no chemical separation), while in set 2 all plutonium isotopes were measured in all 26 smears. To address this experimental situation the complete sets of statistical analyses were repeated for three detection limits. First, the detection limit was set equal to $0.05 \%$ of the gross alpha. This limit corresponds to the lowest measured plutonium to gross alpha ratio in set 2 . The second detection limit was chosen at $0.5 \%$ of gross alpha, which corresponds to typical measurement results obtained in set 2 . In the third case we used the $1 \%$ detection limit, which coincides with precision typically accepted in alpha spectrometry. The latter choice is very pessimistic, and there exist cases in set 1 (see Table A1) where the reported plutonium to gross alpha ratio is less than $1 \%$. Over $70 \%$ of the proportion TRU data points were censored, mostly due to inability to detect $\mathrm{Cm} 246$, a difficult isotope to measure. 
Hence the importance of developing upper and lower bounds for the proportion TRU was increased under these measurement conditions. Of particular interest in this report is a description of the methodologies recommended for computing isotopic proportions, for dealing with censored data, and for rejecting outlier data points. In addition, regression analyses were performed to test the hypothesis that the proportion TRU is independent of gross alpha activity. Finally, the application of the proportion TRU to its utility in the calibration of nondestructive assay instruments is described. We did not reveal any practical discrepancy between the analyses performed with 3 different detection limits for plutonium. Therefore, only the results for the first choice $(0.05 \%)$. were reported in the text. The results of the analyses for the other cases are available upon request. Three concluding plots (Figs. 10b, c, and d) contain information about all three cases to give a graphical impression of how similar the results are.

Of no surprise was the fact that the ratio of $\mathrm{Cm} 244$ alpha activity to gross alpha activity was greater than 0.90 in $93 \%$ of the observations. We found that the best estimate of the proportion TRU is determined by treating the data as lognormally distributed assuming interval censoring for the proportion TRU that was derived using the lower and upper bounds for the proportion TRU when nondetects were present. The mean estimate and its lower and upper bounds were computed according to our developed censoring methods. Special considerations were used for treating each of the two sample sets together, along with outlier observations, and between different waste materials. For a lognormal distribution, the statistically best estimate for proportion TRU is 0.0227 with a bounded range of $[0.0180,0.0267]$, (see Table $5(\mathrm{~b}), \mu_{4}, \bar{\mu}_{7}, \mu_{7}$ ). The lower and upper bounds are quite tight about the mean because there simply is not a large difference in treating an isotope that contributes to proportion TRU as zero versus detection limits. These results are compatible with documented process knowledge; the contribution of alpha activity from TRU radionuclides is very small, with the overwhelming majority of alpha radioactivity from $\mathrm{Cm} 244$. As one would expect, for the smears collected, the proportion of alpha activity that is TRU was not statistically significantly different for various materials, although this inference was based on minimal data for some material types. 
Outlier observations were few in number, but quite pronounced since they contained anywhere from 3 to 5 orders of magnitude more alpha activity than the general cluster of smear data. For set 1 , outliers were from smears of cell sumps, where a large portion of radioactivity can be expected to migrate. For set 2, outliers were from the leachate results. The high activity content on these observations was expected as well. When the outlier observations are included in the combined proportion TRU, the estimate increases a small amount, from 0.0227 to 0.0230 (Table 5(a)), in spite of a 5-magnitude increase in alpha activity for the outliers.

A linear regression showed that the proportion TRU decreases with increasing alpha activity. The following "linear" relationship (Table 6):

$$
\begin{aligned}
& \ln \left(\frac{\text { TRU Activity }}{\text { gross } \alpha \text { activity }}\right)=1.33-0.19 \ln \text { (gross } \alpha \text { activity) [upper bound] } \\
& \ln \left(\frac{\text { TRU Activity }}{\text { gross } \alpha \text { activity }}\right)=1.01-0.24 \ln \text { (gross } \alpha \text { activity) [lower bound] }
\end{aligned}
$$

We must emphasize that while the relationship reported is statistically significant, in practice, it may not be important. The reason for this is two-fold: a) the results are very sensitive to how the large alpha activity results are treated mathematically (i.e. the gross alpha activity between smears and leach results are not 1:1); and b) the error of each data point, i.e. proportion TRU, is very large relative to the change in slope. That is to say, the relative change in slope is negligible relative to the Estimated Observational Error (EOE) for each of the data points. Additionally, we can state that while the amount of TRU activity increases with gross alpha activity, it increases at a smaller rate than the pronounced increase in gross alpha activity; thus leading to a negative relationship when expressed as proportion TRU.

When duplicate smears were made of the same item, the difference between measured activities was negligible compared to variations induced by sampling. In the case where an item (metal or glass) was smeared and then leached, the proportion TRU was within a few percent, even though the absolute alpha activity of the leach test was much larger.

The scope of this project offered a start at how these data might be used for 
application to nondestructive assay. The variable of most interest for TRU waste is the TRU activity concentration, in nanocuries per gram of waste. This is the variable which is to be derived, in bulk, by nondestructive assay instrumentation. As mentioned, two leach tests were run: one on a metal tool, the other on three glass bottles. Leach results provide a more accurate measurement of total radioactivity per item, than do smear results. Dividing by the weight of the leached items gives one an estimate of TRU concentration "for that item". For the 3 glass bottles that were leached, the alpha activity concentration was $4 \mathrm{E}+04 \mathrm{nCi} / \mathrm{g}$ and the TRU concentration 496 $\mathrm{nCi} / \mathrm{g}$ (see Table A3) - the proportion TRU was 0.0124 . Smears were also collected on the glass bottles prior to leaching, yielding a gross alpha of $2.7 \mathrm{E}+04 \mathrm{nCi}$, each. Hence we now have one data point that allows us to express proportion TRU as a function of total alpha concentration and a conversion factor for relating gross alpha to nondestructive assay. By nondestructive assay measurements, gross alpha activity can be estimated, thereby providing an estimate of proportion TRU and hence, the TRU activity concentration ( $\mathrm{nCi} / \mathrm{g}$ ). A link between sampling results and nondestructive assay has been established.

This report takes an exhaustive look at the treatment of currently available data where the desired signal (TRU radioactivity) is a small fraction of the total alpha radio activity. Handling of censored or nondetect observations, pooling data, statistical methods of estimation of isotopic proportions, and performing linear regression are covered in detail. The results of this work were well within nominal expectation. We achieved what we set out to do and now have a better understanding how the use of sampling data are to be integrated into a nondestructive assay program. 


\section{Introduction}

\subsection{Statement of the problem}

A number of regulatory-based drivers have imposed stringent requirements on the characterization of radioactive waste; see $[1,2,3]$. For transuranic (TRU) waste, the Department of Energy's (DOE) Waste Isolation Pilot Plant (WIPP) requires, at a minimum, that the waste generator (a) provide methods for sampling and analyzing waste streams to establish an acceptable-knowledge baseline [4], (b) develop a recurring sampling plan that will assure that the data quality objectives can be met, and (c) calibrate and use nondestructive assay (NDA) instruments for certifying the waste, by bulk measurement, to the WIPP waste acceptance criteria (WAC) [5]. In order to meet these requirements for shipment of TRU waste to WIPP, existing waste characterization methods must be tested, evaluated, improved, and then ultimately approved by DOE and the Environmental Protection Agency (EPA).

Along with the technical and compliance related issues, cost is an important consideration in the identification of radioactive waste as low-level or TRU. This is due to the highly disparate disposal costs for low-level waste versus TRU waste. Current estimates suggest that the disposal costs for TRU waste may be a factor of 10 greater than that of low-level waste per unit volume. Thus, it is very critical - during the waste characterization process - that the radioactive waste be accurately identified as low-level or TRU to avoid any unnecessary cost penalties, while assuring compliance with regulations. Solid waste generated at the Radiochemical Engineering Development Center (REDC) located at Oak Ridge National Laboratory (ORNL) is of particular interest because it has produced approximately $90 \%$ by volume of the historic (legacy) remote-handled (RH) TRU waste, and all of the newly generated RH TRU waste generated at ORNL. Solid radioactive waste from REDC is currently managed as TRU waste, in spite of the fact that the largest fraction of radioactivity is due to the presence of Cm244 and Cr252, isotopes not considered TRU radionuclides [2]. TRU radionuclides of $P u$ and Am are known to be present in the waste, but there has not been satisfactory information in the past to conclusively demonstrate that the TRU concentration is less (or 
greater) than the $100 \mathrm{nCi} / \mathrm{g}$ threshold [DOE Order 5820.2A]. It is imperative that a determination be made regarding the correct classification of REDC wastes in order to ensure the REDC correctly packages its TRU waste to meet the Waste Isolation Pilot Plant (WIPP) WAC [3].

The main objective of this work was to obtain better process knowledge of elemental and isotope structure of solid waste currently handled at REDC. The approach is essentially based on the application of various statistical techniques in the analysis of the collected radiochemical measurements.

The corresponding statistical information, combined with physical knowledge of the processes generating these wastes, allow us to enhance the accuracy and precision of the nondestructive waste characterization based on the technologies developed by the Applied Radiation Measurements Department (ARMD), including Active - Passive Neutron Examination Assay (APNea) and Gamma Active Segmented Passive (GASP); see $[6-10]$.

If we know exactly the isotopic profile for the considered waste stream, then a reliable measurement of the total activity, or some individual components (e.g. gamma emitting isotopes), makes it possible to calculate the contents of all components of interest. The current situation is far from the above one; all components of interest are not known. Of course, waste inputs are stochastic in most respects and even routine inputs are poorly understood (compare with [10]). For instance, the solid waste streams may be "cross-contaminated" by items from another campaign's ${ }^{2}$ "activities; operational upsets can occur; the variation in composition of material after processing in REDC may depend upon operator's action; sampling measurements include a relatively small fraction of the items from a waste stream. Therefore, the main emphasis in this work is done on the statistical description and analysis of the isotope profiles. Sampling experiments are confined to the waste stream generated during Campaign 69.

\footnotetext{
${ }^{2}$ A campaign at the REDC is the duration of a process to separate products from a particular target specification. Campaigns normally run from 1 to 2 years.
} 


\subsection{Material exposition}

We start in Section 2 with a short description of waste generating processes and the main components (elemental or material) occurring in the considered waste stream. A thorough description may be found in [12]. Section 3 outlines the analytical methods used in this work to determine the individual isotopic activities. Section 4 contains an exploratory data analysis, which is based on visualization of available data. Various scatter plots and some simple statistical calculations help to detect a number of unusual entries and outlier observations, and to formulate the statistical hypotheses and models to be tested or verified.

After graphical summaries and evaluations, we proceed in Section 5 with several statistical methods and models to estimate the proportion TRU. The reason for considering many underlying models and methods was to assure the robustness of the final inferences with respect to the assumptions.

Appendix 1 contains a summary of the empirical information used in this study. The technical derivations and mathematical details are reported in two subsequent appendices. In particular, Appendix 2 provides the derivation of maximum likelihood estimators which should be used in the spectrometry to obtain individual isotopic activities. Finally, Appendix 3 focuses on the development of statistically efficient estimators of the proportion TRU under various assumptions concerning the random structure of the waste generation process and the associated observations.

\section{Generating Processes and Main TRU Components.}

During Campaign 69, the REDC processing facilities recovered and purified a number of elements from targets irradiated in the High Flux Isotope Reactor (HFIR) and remainders from past campaigns, for instance, the Savannah River Plant Reactor (SRPR); see $[11,12]$ for details. The list of potential radioactive elements is reported in Table 1.

Our work focused on plutonium, americium, curium, and californium isotopes. While Pu238/239/240, Cm246, and Am241/243 are by definition TRU radionuclides see [21], Cm244 and Cf252 are the primary isotopes (by activity) of REDC wastes. 
Table 1: Potential Radionuclides in REDC Solid Wastes

$\begin{array}{cc}\text { Alpha } & \text { Beta/gamma } \\ \text { emitters } & \text { emitters }\end{array}$

$\begin{array}{ll}\text { Cm242 } & \text { Cs134 } \\ \text { Cm243 } & \text { Cs136 } \\ \text { Cm244 } & \text { Cs137 } \\ \text { Cm245 } & \\ \text { Cm246 } & \text { Bk249 } \\ \text { Cm248 } & \end{array}$

$\begin{array}{ll}\text { Cf249 } & \text { Eu152 } \\ \text { Cf250 } & \text { Eu154 } \\ \text { Cf252 } & \\ \text { Cf253 } & \text { Pm147 } \\ & \text { Ni63 } \\ \text { Ac227 } & \text { Fe55 } \\ & \text { Co60 } \\ \text { Am241 } & \text { I129 } \\ \text { Am242m } & \text { Tc99 } \\ \text { Am243 } & \text { Sm151 } \\ & \text { Sr90 } \\ \text { Ra226* } & \text { Y90 } \\ & \text { Sb125 } \\ \text { Th228* } & \\ \text { Th229* } & \text { Ru106 } \\ \text { Th232* } & \text { Rh106 } \\ & \\ \text { Pu238 } & \text { Ce144 } \\ \text { Pu239 } & \text { Pr144 } \\ \text { Pu240 } & \\ \text { Pu241 } & \\ \text { Pu242 } & \end{array}$

* These radioisotopes are not subject to destructive analysis since radium targets are not currently processed at REDC. 
Actually, the highly prominent presence of Cm244 and Cf252 makes the determination of TRU or not TRU by nondestructive assay (NDA) difficult, since one has to estimate a signal (emission produced by TRU waste elements) which constitutes a small fraction (usually less than $5 \%$ in samples available in this work) of the total signal.

For making the decision as to whether the waste is TRU or not TRU, NDA becomes technically admissible only when there is some prior information about the isotopic profiles of the basic elements. The immediate objective of this work was to provide this important information.

Physically, REDC wastes are very diverse and include, for instance, high effciency particulate air filters from off-gas cleanup systems, gauges, discarded equipment, polyethylene bottles and smaller items from cell wastes. At present, the main concern is the characterization of a legacy population of $208 \ell$ (55 gallons) drums, which are primarily loaded with cell wastes. Therefore, our efforts were directed to measurements with this latter type of wastes. The waste has the composition as described in Table 2.

Table 2: Waste Composition

Waste type
\begin{tabular}{|l|l|c|}
\hline Plastic & Melted poly bottles, poly blocks, MSM boots & $\approx 40 \%$ \\
Glass & Sample bottles & $\approx 30 \%$ \\
Metals & Tools & $\approx 20 \%$ \\
Cloth & Wipes & $\approx 10 \%$ \\
\hline
\end{tabular}

\section{Sampling and Analytical Methods}

\subsection{Sampling}

The selection of the sampling and analytical methods was based on the obvious compromise required between precision, time, and cost effectiveness. Most of the sampling was based on smears (relatively cheap, but not a very precise method). In the case of smears, we have a very significant contribution to the variability of the results because the smearing process itself adds to the total uncertainty through the selection and cov- 
erage of smeared areas. Leaching (precise and expensive) was used only for "control" type sampling. Leaching results are valuable because they actually quantify the total activity on a waste item, allowing us to make the determination of whether or not the waste item(s) is TRU (i.e., greater than the defined limit of $100 \mathrm{nCi} / \mathrm{g}$ ).

Due to the very radioactive nature of the operations, all samples were collected remotely. Smears were taken using. “Q-tip” swabs with wooden handles. After smearing any surface, the $Q$-tip head was placed in a labeled sample bottle, the extra wood broken off, and the bottle capped and removed from the REDC cell bank. This procedure made it possible to avoid contamination from the manipulator fingers and other sources. In the case of poly bottles, the inside of the bottle was smeared as well as the threads. In general, whenever it was possible, an operator tried to smear the most typical elements of every item.

Two sets of samples were collected for this study: 34 smear samples were taken for the first set, 26 smear and two leach samples were taken for the second set. The first set of samples was collected from waste items located in cell 9, where the waste is bagged out, and various locations in cells $1,2,3,4,6,7$, and 8 of the REDC facility. Cell 5 was not used in Campaign 69; therefore, no samples were taken from this cubicle. The second set of samples was taken from waste items in cell 9. Detailed sample locations and the sample tracking records are provided in Tables A4 and A5 of Appendix 1.

Waste items from each waste category were collected from Campaign 69 waste. Each waste item was selected at random from different poly buckets to ensure that the analytical results obtained from these samples represent the TRU wastes.

\subsection{Analytical methods}

The following methods were utilized in analyzing the "Q-tip" smears of waste for radionuclide content. In the first set, samples were analyzed using gross alpha, alpha spectrometry, gamma spectrometry, and gross neutron determination. Gross neutron determinations were performed in order to calculate Cf252 activities. The smears were individually leached in a $4 \mathrm{~N}$ Nitric Acid solution for 2 hours at room temperature. The resulting leachate was made to a known volume prior to analyzing so that a total 
smear activity determination could be made. Gamma spectrometry determinations were made by counting an aliquot of the leachate with a High Purity Germanium detector. The discrete gamma-ray energy peaks were identified and integrated to quantify the gamma-emitting radionuclides; compare with [13]. All of the fission and activation products identified in the smear samples were analyzed by gamma spectrometry, as well as Am241 and Am243. Although the two isotopes of americium are primarily alpha emitters, they were in general very small percentages of the total alpha activity and therefore could not be accurately measured using alpha spectrometry. Their concentrations were determined using gamma-ray spectrometry. The gross alpha determinations were made by evaporating an aliquot of the leachate on a stainless steel plate (in duplicate) followed by alpha counting on a gas proportional counter. One of these plates was then counted in an alpha spectrometer to identify the alpha-emitting radionuclides by pulse-height analysis. In order to quantify the major alpha emitters, each peak (energy) identified by alpha spectrometry was integrated to determine the individual peak areas. Once all peaks were integrated, the areas were summed to provide a total area. The individual peak areas were then divided by the total area to determine the percentage of alpha counts due to a particular alpha energy; compare with $[14,15]$. The gross alpha and alpha spectrometry analyses thus provided quantitative activities for the different alpha energies. From the statistical point of view, this procedure may be considered as the first order approximation of the maximum likelihood estimator (see Appendix 2). The use of the exact maximum likelihood estimator may lead to a significant increase in peak area accuracy. The corresponding statistical software can be developed and applied at later stages of the TRU project.

Once compiled, additional information from gross neutron determinations (Cf252) and gamma ray analyses (Am isotopes) was used to determine the amount of contributions these nuclides made to their respective alpha energy peaks. As an example, $\mathrm{Pu} 238$ and Am241 both have primary alpha energies of nominally $5.50 \mathrm{MeV}$. By quantifying Am241 through gamma spectrometry, the activity contributed by Am241 to the 5.50 $\mathrm{MeV}$ peak was subtracted from the total activity at that energy to leave the Pu238 activity. Alpha-emitting radionuclides that were not registered by alpha spectrometry 
were stated in data sets to be less than $1 \%$ of the total alpha activity. The selection of this detection level was based on the sensitivity of the alpha spectrometer used for the analysis.

Since this project was intended to profile alpha-emitting nuclides in the REDC waste stream (particularly TRU nuclides), no additional radiochemical separations were performed in order to quantify certain pure or nearly pure beta-emitters such as Ni63 and Sr90 that are also known to be present in the waste.

With the first sample set complete, it was apparent that the plutonium isotopes were often not detected by this method of analysis. It was agreed that an additional set of smears ( 26 samples) and two leached samples would be taken and, in addition to the analyses requested for the first sample set, a plutonium separation would be performed. The plutonium was removed from an aliquot of leachate by solvent extraction and then analyzed through a separate gross alpha and alpha spectrometry determination as described previously. Thus, plutonium isotopes of interest (Pu238,239,240,242) were separated from the other alpha emitters so that the limits of detection were greatly reduced, at least by an order of magnitude. This series of samples was complemented by two leaching experiments, in which the items previously smeared were used. All the data are compiled in Appendix 1.

\section{Exploratory Analysis}

\subsection{Data description and notations}

The analysis of the data has focused on the activity (Bq/total) observations for gross alpha and a collection of alpha-emitting radionuclides. Each observation vector from a given sample, which consisted of the observed activities with associated errors from a sample, was identified according to the cell source and waste type (i.e., plastic, metal, glass, etc.). The following alpha-emitting radionuclides were found: Am241, Am243, Cf252, Cm242, Cm244, Cm246, Pu238, and Pu239/240. The TRU activity for each sample was calculated from the sum of the activities of the following radionuclides: Am241, Am243, Cm246, Pu238, and Pu239/240. As mentioned in the previous section, 
the data were obtained from two separate sample sets. The first sample set consisted exclusively of smear samples and a plutonium separation was not performed. The second sample set, in addition to smear samples, also had two leaching samples, on which plutonium separations were performed. The second sample set was required because of the high proportion of nondetects in the first sample set, i.e., observed activities known only to be "below a detection limit." A number of statistical and graphical methods were applied to the two data sets (34 observations in the first and 28 observations in the second). These methods were directed toward:

1. the detection of outliers or unusual observations;

2. a search for inconsistent observation vectors (e.g., individual radionuclide activities sum to a value greater than the gross alpha activity);

3. the analysis of observation vectors with nondetects; and

4. the graphical analysis of the proportion of TRU activity by waste type.

The isotopic ratios or proportions for the radionuclides of interest were calculated from each smear using the ratio of the observed radionuclide activity to the sample average gross alpha activity. Because the primary interest was in the TRU activity and its proportion, the analysis focused on the following components or proportions:

1. TRU (PTRU) (consists of Cm246, Pu238, Pu239/240, Am241,Am243);

2. $\mathrm{Cm} 244$ (PCm244);

3. $\mathrm{Cf252}$ (PCf252);

4. the remaining radionuclides REST (PREST);

5. gross alpha (GA).

(The "P" in the notation for the above components means proportion, e.g., PTRU is the proportion of TRU activity in the sample.) We used the compound variables (i.e., aggregated radionuclide activities) to reduce the impact of the large number of nondetects. Actually, introduction of the variable REST is redundant in this particular 
setting, because it comprises only one measurable radionuclide $\mathrm{Cm} 242$ (activities of other radionuclides listed in Table 1 were negligible). However, we use this notation to emphasize the idea of aggregation, which can be employed in similar situations but with a larger number of radionuclides. In what follows, we refer to set 1 as 34 samples from the first data set, and to set 2 as samples from the second data set.

Appendix 1 contains available information about these two data sets. It is evident that the number of nondetects, especially in set 1 , is too large to use statistical approaches that do not account for this. Therefore, we had to apply alternative statistical procedures, which were developed to handle the nondetects properly. They are computationally intensive and demand some mathematical rigor. Before we proceed with those procedures, let us explore the data by relying upon visualization techniques.

\subsection{Nondetects handling}

As mentioned above, for many of the samples there were activities for some radionuclides (e.g., Cm246) known only to be below a detection limit, which was taken as $1 \%$ of the mean gross alpha activity. This bound is based on limitations of the analytical technique (see Section 3.2). However, for the plutonium radionuclides, the second set, which incorporated plutonium separations prior to analysis, showed that none of the samples contained individual plutonium radionuclides at a level lower than $0.05 \%$ of the gross alpha. Therefore, for statistical analyses, the lower limit of detection for these nuclides was set to $0.05 \%$ of the gross alpha (see comments about analyses for other choices of detection limits in the Executive Summary). A censoring flag (CNS) was defined for each radionuclide that equaled 0 for a detect and equaled 1 if the observed activity was a nondetect. For a nondetect, the activity and activity error were set equal to each other and this is what was used to designate a nondetect observation. Because of the presence of nondetects, the calculated TRU activity from the sum of the stated activities for the five radionuclides mentioned above actually represents an upper limit on TRU activity whenever nondetect components are present. A lower limit on the TRU activity was also calculated by replacing a nondetect TRU radionuclide component by 0 . See the next section for more details. 


\subsection{Visual analysis}

The observed activities for the radionuclides of interest ranged over orders of magnitude. Therefore, subsequent graphs and analyses were generally performed using the natural logarithm of the observed activities. The following plots were used in the preliminary evaluation of both data sets. Figures $1(\mathrm{a}-\mathrm{c})$ and $2(\mathrm{a}-\mathrm{c})$ show the transformed TRU, Cf252, and Cm244 versus the transformed gross alpha activity for each experiment. The " $x$ "'s in these figures indicate nondetect observations. In the case of TRU, it means that at least one of the TRU radionuclides was not detected. In this work, we have not explicitly addressed the problem of different numbers of nondetects in the compound variables. Some conclusions from a review of these figures are:

1. The significant number of nondetects (see last two columns in Tables 3-5 for exact numbers) will cause difficulties in the estimation of the TRU isotopic ratio in both sample sets (Figures $1 \mathrm{a}$ and $2 \mathrm{a}$ ).

2. Cf252 and Cm244 activities do not have a significant problem with nondetects (Figures $1 b$ and $2 b$ ).

3. Cm244 activity is the dominant activity in each sample. This can be seen in Figures $1 \mathrm{c}$ and $2 \mathrm{c}$ where most of the observations fall close to the $45^{\circ}$ line $y=x$, meaning that the $\mathrm{Cm} 244$ activity is nearly equal to the gross alpha activity.

4. There are a couple of observations in each experiment that fall away from the bulk of the data, usually at the high gross alpha levels. We call these points leverage points and they are discussed in more detail later.

Figures 3 (a-c) and $4(\mathrm{a}-\mathrm{c})$ are similar to Figures 1 and 2 except that "G" stands for glass, " $\mathrm{M}$ " for metal, " $\mathrm{P}$ " for poly (melted or bottle), " $\mathrm{C}$ " for cell, and " $\mathrm{W}$ " for wipes.

Additional information becomes more illuminating if we introduce upper and lower bounds on an observed compound variable (see [16]), such as TRU activity, where for the $i$-th observation the upper bound is given by

$$
u_{i}=\sum_{j=1}^{k} y_{i j}
$$


where $j$ stands for radionuclide type, $k$ is the number of radionuclides in the corresponding compound variable, $y_{i j}$ is either an observed activity value, or a detection limit, discussed previously in Section 4.2. The lower bound is given by

$$
\ell_{i}=\sum_{j=1}^{k^{\prime}} y_{i j}
$$

where $k^{\prime}$ includes only the observed radionuclide activities in the compound variable, i.e., nondetects are omitted. "Two-sided" information is presented in Figures 5 and 6. We use " 0 " when $u_{i}=\ell_{i}$ (i.e., when there are no nondetects), and $U$ or $L$ to distinguish the upper and lower limits, respectively, when $u_{i} \neq \ell_{i}$.

Additional facts may be derived from a review of Figures 1 - 6 .

1. There are several points, which stand apart from the majority, primarily at the highest activity levels. These are, so called, leverage points (see Figures la and 2a) and must be treated with some caution since these observations may dominate and distort any estimates or conclusions. Because of the extremely high gross alpha activity in two samples from each experiment (ID $=$ RH4D and RH8A in sample set 1 and ID = LN-GL and LN-ML, the leached samples in sample set 2), the analyses were performed with and without these points to evaluate their effect on the results. RH4D was a smear from the sump in cell 4; RH8A from the sump in cell 8 . The leached samples were glass bottles (GL) and metal (ML).

2. Two points (ID = RH4D and RH8A in sample set 1 ) correspond to smears made on the cell's surface. Actually, all observations of this type must be treated with some extra caution, because they can be affected more than others by previous campaigns. An attentive reader may notice that the majority of results marked with " $\mathrm{C}$ " in Figure 3 (a-c) stand apart from the rest of the data.

3. Observations from sample set 2 generally have higher gross alpha activities than those in sample set 1 . The explanation could be very simple: two different operators collected samples for these two sets. Perhaps the second one spent more time in "smearing" items. However, other causes may be involved as well, for 
example process changes during periods between the sampling times.

4. Observations are generally scattered along the straight line with unit slope. What could be the cause of that? For example, let us assume that ( $i$ stands for the observation number):

$$
\frac{T R U_{i}}{G A_{i}} \equiv \text { const }=f
$$

Then

$$
\ln T R U_{i}=\ln f+\ln G A_{i}
$$

which can be written as $y_{i 1}=c+y_{i 2}$ using the notation introduced in the comments to (1). Thus, the observed phenomenon is rather strong evidence that the ratio of activities of TRU and Cm244 is stable but affected by various "random" factors. More thorough analysis of this statement will be postponed until the following section.

\section{Estimation of TRU Isotopic Ratio}

\subsection{Analysis of sample means}

As an additional analysis of the evaluation of the radionuclide activities, different underlying mathematical models were used to estimate the mean PTRU in each experiment by waste type as well as combined. Only the individual TRU isotopic ratio from each sample, i.e., ratio of individual isotopic peak area to total peak area, as available for the analysis (see Section 3.2 for details). If the individual isotopic peak areas become available a more efficient statistical procedure, developed in the framework of this study and described in Appendix 3, could be used to evaluate PTRU and other isotopic ratios.

All of the models in this analysis made use of the nondetect or censored observations using both parametric and nonparametric methods. The basic parametric models assumed an underlying censored normal or lognormal distribution for the observed proportions. Different censoring mechanisms were also considered, e.g., one-sided or interval censoring. In the one-sided interval censoring case, the censored or nondetect observations are assumed to be less than the specified detection limit, i.e., the censored value could be any value from minus infinity to the detection limit (see $\hat{\mu}_{1}, \hat{\mu}_{2}$ and $\hat{\mu}_{5}$ in 
Tables 3-5). In the closed interval censoring case, the censored nondetect observations are assumed to be between zero and the detection limit (see $\hat{\mu}_{3}$ and $\hat{\mu}_{4}$ in Tables 3-5). The nonparametric methods made use of estimates originally developed in the analysis of censored survival times. The nonparametric method uses the product limit estimate of the cumulative distribution function to obtain the estimated mean. The estimate was originally given by Kaplan and Meier and is described in [16]. All computations to obtain the censored estimates are based on procedures from the Statistical Analysis System (SAS [17]).

Tables 3,4 , and 5 provide a summary of the estimates of the mean of PTRU from the parametric and nonparametric models for the two sample sets individually and combined. The normal method noted in these tables assumes the original untransformed observations are normally distributed, whereas the lognormal method assumes the logtransformed observations are normally distributed. It should be emphasized that the Kaplan-Meier estimate does not need any assumptions about normality. Because of the $100 \%$ nondetects for the glass and metal components in sample set 1 and the poly component in sample set 2 , no reliable estimates are available for these materials. When there is only one detect in the sample, the nonparametric method (Kaplan-Meier) cannot provide a standard error estimate in this case. The nonparametric method requires fewer assumptions than the parametric methods, and, therefore, gives us more defensible estimates; see [16]. However some of the parametric methods provide estimates of similar magnitudes. If additional sampling is planned, it would be advisable to obtain more detect observations from each waste component. A rough rule-of-thumb would be that if we desire a $50 \%$ reduction in the standard error, we should quadruple the sample size.

The first two parametric estimates reported in all tables are based on one-sided censoring, i.e. in the analyses we used only the fact that (see (1) in Section 4)

$$
T R U_{i} \leq u_{i}
$$

As it can be seen, the use of only this fact generally leads to estimates (i.e., $\hat{\mu}_{1}, \hat{\mu}_{2}$ ) 
which are unrealistically low, and must be discarded in future studies. As soon as we complemented (5) by an obvious constraint that

$$
\ell_{i} \leq T R U_{i}
$$

all of the estimates reached the vicinity of 0.03 for the first sample set and 0.02 for the second one (see estimates $\hat{\mu}_{3}$ and $\hat{\mu}_{4}$ ).

The last two estimates (i.e., "Normal sample mean" and "Lognormal sample mean") in Tables 3 - 5 are determined as follows:

$$
\bar{\mu}_{6}=n^{-1} \sum_{i=1}^{n} u_{i} \text { and } \mu_{6}=n^{-1} \sum_{i=1}^{n} \ell_{i},
$$

$\bar{\mu}_{7}$ and $\underline{\mu}_{7}$ are the same as $\bar{\mu}_{6}$ and $\underline{\mu}_{6}$ except that the logarithm of the observed activities are used rather than the untransformed activities. If $\hat{\mu}$ is a hypothetical arithmetic mean constructed under the assumption that all nondetects are known, then obviously

$$
\underline{\mu}_{6} \leq \hat{\mu} \leq \bar{\mu}_{6}
$$

One can see that the discrepancy between $\underline{\mu}_{6}$ and $\bar{\mu}_{6}$ in all the tables is of order 0.01 or less in spite of a large number of nondetects the "true" but unknown $\hat{\mu}$ falls within a $30 \%$ range. The estimates which take into consideration the interval censoring (they are marked in the tables by the "Type Cens" entry "Interval") are regularly included in the interval $(\mu, \bar{\mu})$. For instance, in all tables

$$
\underline{\mu}_{6}<\bar{\mu}_{3}<\bar{\mu}_{6}
$$

Tables 3, 4, and 5 are the results of the statistical analysis of set 1 , set 2 , and sets 1 plus 2, respectively. The tables are partitioned into (a) and (b), corresponding to all observations and outlier observations omitted. The columns denote the method of estimation; the type of data censoring employed; the waste component type (wipes, poly, metal, glass, cell, or combined); the mean estimate for PTRU, the proportion TRU relative to gross alpha radioactivity; the standard error of the mean for PTRU; 
TABLE 3(a). ESTIMATION OF TRU PROPORTION FROM FTRT SAMPLE SET NO OBSERVATIONS OMTTIED

\begin{tabular}{|c|c|c|c|c|c|c|}
\hline Method & Type Cens & Waste Comp & Estimate & Std Ent & $n$ & *Cens \\
\hline $\begin{array}{l}\text { Normal } \\
\mu_{1}\end{array}$ & One-sided & $\begin{array}{l}\text { Combined } \\
\text { Cell } \\
\text { Glass } \\
\text { Metal } \\
\text { Poly } \\
\text { Wrpes }\end{array}$ & $\begin{array}{c}0.0005 \\
-0.0005 \\
- \\
- \\
0.0044 \\
0.0220\end{array}$ & $\begin{array}{c}0.0128 \\
0.0215 \\
- \\
- \\
0.0147 \\
0.0141\end{array}$ & $\begin{array}{c}34 \\
9 \\
5 \\
4 \\
11 \\
5\end{array}$ & $\begin{array}{c}30 \\
8 \\
5 \\
4 \\
10 \\
3\end{array}$ \\
\hline $\begin{array}{l}\text { Lognormal } \\
a_{2}\end{array}$ & One-sided & $\begin{array}{l}\text { Combined } \\
\text { Cell } \\
\text { Gluss } \\
\text { Meal } \\
\text { Poly } \\
\text { Wipes }\end{array}$ & $\begin{array}{c}0.0088 \\
0.0072 \\
- \\
- \\
0.0064 \\
0.0240\end{array}$ & $\begin{array}{c}0.0042 \\
0.0072 \\
- \\
- \\
0.0060 \\
0.0085\end{array}$ & $\begin{array}{c}34 \\
9 \\
5 \\
4 \\
11 \\
5\end{array}$ & $\begin{array}{c}30 \\
8 \\
5 \\
4 \\
10 \\
3\end{array}$ \\
\hline $\begin{array}{l}\text { Nomnl } \\
\mu_{3}\end{array}$ & Interval & $\begin{array}{l}\text { Combined } \\
\text { Cell } \\
\text { Glaes } \\
\text { Metal } \\
\text { Poly } \\
\text { Wipes }\end{array}$ & $\begin{array}{l}0.0359 \\
0.0331 \\
0.0617 \\
0.0341 \\
0.0291 \\
0.0313\end{array}$ & $\begin{array}{l}0.0046 \\
0.0132 \\
0.0132 \\
0.0021 \\
0.0036 \\
0.0061\end{array}$ & $\begin{array}{c}34 \\
9 \\
5 \\
4 \\
11 \\
5\end{array}$ & $\begin{array}{c}30 \\
8 \\
5 \\
4 \\
10 \\
3\end{array}$ \\
\hline $\begin{array}{l}\text { Lognomind } \\
\mu_{4}\end{array}$ & Interval & $\begin{array}{l}\text { Combined } \\
\text { Cell } \\
\text { Glass } \\
\text { Metal } \\
\text { Poly } \\
\text { Wipes }\end{array}$ & $\begin{array}{l}0.0291 \\
0.0217 \\
0.0539 \\
0.0340 \\
0.0259 \\
0.0289\end{array}$ & $\begin{array}{l}0.0032 \\
0.0062 \\
0.0132 \\
0.0020 \\
0.0042 \\
0.0051\end{array}$ & $\begin{array}{c}34 \\
9 \\
5 \\
4 \\
11 \\
5\end{array}$ & $\begin{array}{c}30 \\
8 \\
5 \\
4 \\
10 \\
3\end{array}$ \\
\hline $\begin{array}{l}\text { Kaplan-Meier } \\
\boldsymbol{A}_{S}\end{array}$ & One-sided & $\begin{array}{l}\text { Combined } \\
\text { Cell } \\
\text { Glass } \\
\text { Metal } \\
\text { Poly } \\
\text { Wipes }\end{array}$ & $\begin{array}{c}0.0274 \\
0.0294 \\
- \\
- \\
0.0257 \\
0.0348\end{array}$ & $\begin{array}{c}0.0012 \\
\dot{-} \\
- \\
0.0069\end{array}$ & $\begin{array}{c}34 \\
9 \\
5 \\
4 \\
11 \\
5\end{array}$ & $\begin{array}{c}30 \\
8 \\
5 \\
4 \\
10 \\
3\end{array}$ \\
\hline $\begin{array}{l}\text { Normal Surnple Mean } \\
\text { (Upper bound) } \\
\bar{\mu}_{6}\end{array}$ & Jgnored & $\begin{array}{l}\text { Combined } \\
\text { Cell } \\
\text { Glass } \\
\text { Meenl } \\
\text { Poly } \\
\text { Wipes }\end{array}$ & $\begin{array}{l}0.0402 \\
0.0373 \\
0.0669 \\
0.0395 \\
0.0332 \\
0.0343\end{array}$ & $\begin{array}{l}0.0048 \\
0.0141 \\
0.0148 \\
0.0029 \\
0.0042 \\
0.0058\end{array}$ & $\begin{array}{c}34 \\
9 \\
5 \\
4 \\
11 \\
5\end{array}$ & $\begin{array}{c}30 \\
8 \\
5 \\
4 \\
10 \\
3\end{array}$ \\
\hline $\begin{array}{l}\text { Normal Sumple Mean } \\
\text { (Lower bound) } \\
\underline{\mu}_{6}\end{array}$ & Set to 0 & $\begin{array}{l}\text { Combined } \\
\text { Cell } \\
\text { Glass } \\
\text { Metal } \\
\text { Poly } \\
\text { Wipes }\end{array}$ & $\begin{array}{l}0.0317 \\
0.0289 \\
0.0566 \\
0.0290 \\
0.0255 \\
0.0278\end{array}$ & $\begin{array}{l}0.0047 \\
0.0140 \\
0.0147 \\
0.0029 \\
0.0032 \\
0.0076\end{array}$ & $\begin{array}{c}34 \\
9 \\
5 \\
4 \\
11 \\
5\end{array}$ & $\begin{array}{c}30 \\
8 \\
5 \\
4 \\
10 \\
3\end{array}$ \\
\hline $\begin{array}{l}\text { Lognormal Sample Mean } \\
\text { (Upper bound) } \\
\bar{\mu}_{7}\end{array}$ & Ignored & $\begin{array}{l}\text { Combined } \\
\text { Cell } \\
\text { Glass } \\
\text { Metal } \\
\text { Poly } \\
\text { Wipes }\end{array}$ & $\begin{array}{l}0.0336 \\
0.0272 \\
0.0597 \\
0.0392 \\
0.0294 \\
0.0327\end{array}$ & $\begin{array}{l}0.0034 \\
0.0067 \\
0.0148 \\
0.0029 \\
0.0052 \\
0.0048\end{array}$ & $\begin{array}{c}34 \\
9 \\
5 \\
4 \\
11 \\
5\end{array}$ & $\begin{array}{c}30 \\
8 \\
5 \\
4 \\
10 \\
3\end{array}$ \\
\hline $\begin{array}{l}\text { Lognormal Sample Mean (Log) } \\
\text { (Lower bound) } \\
\underline{\mu}_{7}\end{array}$ & Set to 0 & $\begin{array}{l}\text { Combined } \\
\text { Cell } \\
\text { Glass } \\
\text { Metal } \\
\text { Poly } \\
\text { Wipes }\end{array}$ & $\begin{array}{l}0.0234 \\
0.0144 \\
0.0479 \\
0.0286 \\
0.0230 \\
0.0245\end{array}$ & $\begin{array}{l}0.0035 \\
0.0064 \\
0.0146 \\
0.0029 \\
0.0036 \\
0.0059\end{array}$ & $\begin{array}{c}34 \\
9 \\
5 \\
4 \\
11 \\
5\end{array}$ & $\begin{array}{c}30 \\
8 \\
5 \\
4 \\
10 \\
3\end{array}$ \\
\hline
\end{tabular}


TABLE 3(2). ESTIMATION OR TRU PROPORTION FROM FIRST SAMPLE SET NO OBSERVATIONS OMITTED

\begin{tabular}{|c|c|c|c|c|c|c|}
\hline Method & Type Cens & Waste Comp & Estimate & Std Ent & $\mathbf{n}$ & \# Cens \\
\hline $\begin{array}{l}\overline{\overline{\text { Normal }}} \\
\hat{\mu}_{1}\end{array}$ & One-sided & $\begin{array}{l}\text { Combined } \\
\text { Cell } \\
\text { Glass } \\
\text { Metal } \\
\text { Poly } \\
\text { Wipes }\end{array}$ & $\begin{array}{c}0.0005 \\
-0.0005 \\
- \\
- \\
0.0044 \\
0.0220\end{array}$ & $\begin{array}{c}0.0128 \\
0.0215 \\
- \\
- \\
0.0147 \\
0.0141\end{array}$ & $\begin{array}{c}34 \\
9 \\
5 \\
4 \\
11 \\
5\end{array}$ & $\begin{array}{c}30 \\
8 \\
5 \\
4 \\
10 \\
3\end{array}$ \\
\hline $\begin{array}{l}\text { Lognormal } \\
\beta_{2}\end{array}$ & One-sided & $\begin{array}{l}\text { Combined } \\
\text { Cell } \\
\text { Glass } \\
\text { Metal } \\
\text { Poly } \\
\text { Wipes }\end{array}$ & $\begin{array}{c}0.0088 \\
0.0072 \\
- \\
-\overline{0} \\
0.0064 \\
0.0240\end{array}$ & $\begin{array}{c}0.0042 \\
0.0072 \\
- \\
-\overline{0} \\
0.0060 \\
0.0085\end{array}$ & $\begin{array}{c}34 \\
9 \\
5 \\
4 \\
11 \\
5\end{array}$ & $\begin{array}{c}30 \\
8 \\
5 \\
4 \\
10 \\
3\end{array}$ \\
\hline $\begin{array}{l}\text { Nomal } \\
A_{3}\end{array}$ & Interval & $\begin{array}{l}\text { Combined } \\
\text { Cell } \\
\text { Glass } \\
\text { Metal } \\
\text { Poly } \\
\text { Wipes }\end{array}$ & $\begin{array}{l}0.0359 \\
0.0331 \\
0.0617 \\
0.0341 \\
0.0291 \\
0.0313\end{array}$ & $\begin{array}{l}0.0046 \\
0.0132 \\
0.0132 \\
0.0021 \\
0.0036 \\
0.0061\end{array}$ & $\begin{array}{c}34 \\
9 \\
5 \\
4 \\
11 \\
5\end{array}$ & $\begin{array}{c}30 \\
8 \\
5 \\
4 \\
10 \\
3\end{array}$ \\
\hline $\begin{array}{l}\text { Lognormal } \\
A_{4}\end{array}$ & Interval & $\begin{array}{l}\text { Combined } \\
\text { Cell } \\
\text { Glass } \\
\text { Metal } \\
\text { Poly } \\
\text { Wipes }\end{array}$ & $\begin{array}{l}0.0291 \\
0.0217 \\
0.0539 \\
0.0340 \\
0.0259 \\
0.0289\end{array}$ & $\begin{array}{l}0.0032 \\
0.0062 \\
0.0132 \\
0.0020 \\
0.0042 \\
0.0051\end{array}$ & $\begin{array}{c}34 \\
9 \\
5 \\
4 \\
11 \\
5\end{array}$ & $\begin{array}{c}30 \\
8 \\
5 \\
4 \\
10 \\
3\end{array}$ \\
\hline $\begin{array}{l}\text { Kaplan-Meier } \\
\beta_{5}\end{array}$ & One-sided & $\begin{array}{l}\text { Combined } \\
\text { Cell } \\
\text { Glass } \\
\text { Metal } \\
\text { Poly } \\
\text { Wipes }\end{array}$ & $\begin{array}{c}0.0274 \\
0.0294 \\
- \\
0 . \overline{0} 57 \\
0.0348\end{array}$ & $\begin{array}{c}0.0012 \\
\dot{-} \\
= \\
0.0069\end{array}$ & $\begin{array}{c}34 \\
9 \\
5 \\
4 \\
11 \\
5\end{array}$ & $\begin{array}{c}30 \\
8 \\
5 \\
4 \\
10 \\
3\end{array}$ \\
\hline $\begin{array}{l}\text { Nomal Sample Mean } \\
\text { (Upper bound) } \\
\bar{\mu}_{6}\end{array}$ & Ignored & $\begin{array}{l}\text { Combined } \\
\text { Cell } \\
\text { Glass } \\
\text { Metal } \\
\text { Poly } \\
\text { Wipes }\end{array}$ & $\begin{array}{l}0.0402 \\
0.0373 \\
0.0669 \\
0.0395 \\
0.0332 \\
0.0343\end{array}$ & $\begin{array}{l}0.0048 \\
0.0141 \\
0.0148 \\
0.0029 \\
0.0042 \\
0.0058\end{array}$ & $\begin{array}{c}34 \\
9 \\
5 \\
4 \\
11 \\
5\end{array}$ & $\begin{array}{c}30 \\
.8 \\
5 \\
4 \\
10 \\
3\end{array}$ \\
\hline $\begin{array}{l}\text { Normal Sample Mean } \\
\text { (Lower bound) } \\
\underline{\mu}_{6}\end{array}$ & Set to 0 & $\begin{array}{l}\text { Combined } \\
\text { Cell } \\
\text { Glass } \\
\text { Metal } \\
\text { Poly } \\
\text { Wipes }\end{array}$ & $\begin{array}{l}0.0317 \\
0.0289 \\
0.0566 \\
0.0290 \\
0.0255 \\
0.0278\end{array}$ & $\begin{array}{l}0.0047 \\
0.0140 \\
0.0147 \\
0.0029 \\
0.0032 \\
0.0076\end{array}$ & $\begin{array}{c}34 \\
9 \\
5 \\
4 \\
11 \\
5\end{array}$ & $\begin{array}{c}30 \\
8 \\
5 \\
4 \\
10 \\
3\end{array}$ \\
\hline $\begin{array}{l}\text { Lognormal Sample Mean } \\
\text { (Upper bound) } \\
\bar{\mu}_{7}\end{array}$ & Ignored & $\begin{array}{l}\text { Combined } \\
\text { Cell } \\
\text { Glass } \\
\text { Metal } \\
\text { Poly } \\
\text { Wipes }\end{array}$ & $\begin{array}{l}0.0336 \\
0.0272 \\
0.0597 \\
0.0392 \\
0.0294 \\
0.0327\end{array}$ & $\begin{array}{l}0.0034 \\
0.0067 \\
0.0148 \\
0.0029 \\
0.0052 \\
0.0048\end{array}$ & $\begin{array}{c}34 \\
9 \\
5 \\
4 \\
11 \\
5\end{array}$ & $\begin{array}{c}30 \\
8 \\
5 \\
4 \\
10 \\
3\end{array}$ \\
\hline $\begin{array}{l}\text { Lognomal Sample Mean } \\
\text { (Lower bound) } \\
\underline{\mu}_{7}\end{array}$ & Set to 0 & $\begin{array}{l}\text { Combined } \\
\text { Cell } \\
\text { Glass } \\
\text { Metal } \\
\text { Poly } \\
\text { Wipes }\end{array}$ & $\begin{array}{l}0.0234 \\
0.0144 \\
0.0479 \\
0.0286 \\
0.0230 \\
0.0245\end{array}$ & $\begin{array}{l}0.0035 \\
0.0064 \\
0.0146 \\
0.0029 \\
0.0036 \\
0.0059\end{array}$ & $\begin{array}{c}34 \\
9 \\
5 \\
4 \\
11 \\
5\end{array}$ & $\begin{array}{c}30 \\
8 \\
5 \\
4 \\
10 \\
3\end{array}$ \\
\hline
\end{tabular}


TABLE 4(a). ESTIMATION OF TRU PROPORTION FROM SECOND SAMPLE SET NO OBSERVATIONS OMITTED

\begin{tabular}{|c|c|c|c|c|c|c|}
\hline Method & Type Cens & Waste Comp & Estimate & Std Err & $\mathbf{n}$ & \# Cens \\
\hline $\begin{array}{l}\text { Normal } \\
\hat{\mu}_{1}\end{array}$ & One-sided & $\begin{array}{l}\text { Combined } \\
\text { Glass } \\
\text { Metal } \\
\text { Poly }\end{array}$ & $\begin{array}{c}0.0111 \\
0.0134 \\
0.0202 \\
-\end{array}$ & $\begin{array}{c}0.0036 \\
0.0005 \\
0.0034 \\
-\end{array}$ & $\begin{array}{l}28 \\
13 \\
12 \\
3\end{array}$ & $\begin{array}{l}20 \\
12 \\
5 \\
3\end{array}$ \\
\hline $\begin{array}{l}\text { Lognormal } \\
\hat{\mu}_{2}\end{array}$ & One-sided & $\begin{array}{l}\text { Combined } \\
\text { Glass } \\
\text { Metal } \\
\text { Poly }\end{array}$ & $\begin{array}{c}0.0131 \\
0.0134 \\
.0 .0188 \\
-\end{array}$ & $\begin{array}{c}0.0019 \\
0.0005 \\
0.0026 \\
-\end{array}$ & $\begin{array}{l}28 \\
13 \\
12 \\
3\end{array}$ & $\begin{array}{l}20 \\
12 \\
5 \\
3\end{array}$ \\
\hline $\begin{array}{l}\text { Normal } \\
\hat{\mu}_{3}\end{array}$ & Interval & $\begin{array}{l}\text { Combined } \\
\text { Glass } \\
\text { Metal } \\
\text { Poly }\end{array}$ & $\begin{array}{l}0.0197 \\
0.0134 \\
0.0244 \\
0.0322\end{array}$ & $\begin{array}{l}0.0021 \\
0.0005 \\
0.0028 \\
0.0098\end{array}$ & $\begin{array}{l}28 \\
13 \\
12 \\
3\end{array}$ & $\begin{array}{l}20 \\
12 \\
5 \\
3\end{array}$ \\
\hline $\begin{array}{l}\text { Lognormal } \\
\hat{\mu}_{4}\end{array}$ & Interval & $\begin{array}{l}\text { Combined } \\
\text { Glass } \\
\text { Métal } \\
\text { Poly }\end{array}$ & $\begin{array}{l}0.0173 \\
0.0134 \\
0.0225 \\
0.0281\end{array}$ & $\begin{array}{l}0.0017 \\
0.0005 \\
0.0026 \\
0.0085\end{array}$ & $\begin{array}{l}28 \\
13 \\
12 \\
3\end{array}$ & $\begin{array}{l}20 \\
12 \\
5 \\
3\end{array}$ \\
\hline $\begin{array}{l}\text { Kaplan-Meier } \\
\hat{\mu}_{5}\end{array}$ & One-sided & $\begin{array}{l}\text { Combined } \\
\text { Glass } \\
\text { Metal } \\
\text { Poly }\end{array}$ & $\begin{array}{c}0.0157 \\
0.0140 \\
0.0204 \\
-\end{array}$ & $\begin{array}{c}0.0015 \\
- \\
0.0032 \\
-\end{array}$ & $\begin{array}{l}28 \\
13 \\
12 \\
3\end{array}$ & $\begin{array}{l}20 \\
12 \\
5 \\
3\end{array}$ \\
\hline $\begin{array}{l}\text { Normal Sample Mean } \\
\text { (Upper bound) } \\
\bar{\mu}_{6}\end{array}$ & Ignored & $\begin{array}{l}\text { Combined } \\
\text { Glass } \\
\text { Metal } \\
\text { Poly }\end{array}$ & $\begin{array}{l}0.0315 \\
0.0167 \\
0.0266 \\
0.0366\end{array}$ & $\begin{array}{l}0.0021 \\
0.0006 \\
0.0032 \\
0.0115\end{array}$ & $\begin{array}{l}28 \\
13 \\
12 \\
3\end{array}$ & $\begin{array}{c}20 \\
12 \\
5 \\
3\end{array}$ \\
\hline $\begin{array}{l}\text { Normal Sample Mean } \\
\text { (Lower bound) } \\
\underline{\mu}_{6}\end{array}$ & Set to 0 & $\begin{array}{l}\text { Combined } \\
\text { Glass } \\
\text { Metal } \\
\text { Poly }\end{array}$ & $\begin{array}{l}0.0161 \\
0.0075 \\
0.0225 \\
0.0276\end{array}$ & $\begin{array}{l}0.0023 \\
0.0008 \\
0.0028 \\
0.0123\end{array}$ & $\begin{array}{l}28 \\
13 \\
12 \\
3\end{array}$ & $\begin{array}{l}20 \\
12 \\
5 \\
3\end{array}$ \\
\hline $\begin{array}{l}\text { Lognormal Sample Mean } \\
\text { (Upper bound) } \\
\bar{\mu}_{7}\end{array}$ & Ignored & $\begin{array}{l}\text { Combined } \\
\text { Glass } \\
\text { Metal } \\
\text { Poly }\end{array}$ & $\begin{array}{l}0.0211 \\
0.0166 \\
0.0244 \\
0.0333\end{array}$ & $\begin{array}{l}0.0016 \\
0.0006 \\
0.0031 \\
0.0100\end{array}$ & $\begin{array}{l}28 \\
13 \\
12 \\
3\end{array}$ & $\begin{array}{l}20 \\
12 \\
5 \\
3\end{array}$ \\
\hline $\begin{array}{l}\text { Lognormal Sample Mean } \\
\text { (Lower bound) } \\
\underline{\mu}_{7}\end{array}$ & Set to 0 & $\begin{array}{l}\text { Combined } \\
\text { Glass } \\
\text { Metal } \\
\text { Poly }\end{array}$ & $\begin{array}{l}0.0127 \\
0.0070 \\
0.0208 \\
0.0227\end{array}$ & $\begin{array}{l}0.0017 \\
0.0007 \\
0.0024 \\
0.0098\end{array}$ & $\begin{array}{l}28 \\
13 \\
12 \\
3\end{array}$ & $\begin{array}{c}20 \\
12 \\
5 \\
3\end{array}$ \\
\hline
\end{tabular}


TABLE 4(b). ESTMMATION OF TRU PROPORTION FROM SECOND SAMPLE SET TWO OUTLIER OBSERVATIONS FROM LEACHING SAMPLES OMITTED

\begin{tabular}{|c|c|c|c|c|c|c|}
\hline Method & Type Cens & Waste Comp & Estimate & Std Ert & $n$ & \# Cens \\
\hline $\begin{array}{l}\text { Normal } \\
\hat{\mu}_{1}\end{array}$ & One-sided & $\begin{array}{l}\text { Combined } \\
\text { Glass } \\
\text { Metal } \\
\text { Poly }\end{array}$ & $\begin{array}{c}0.0114 \\
0.0134 \\
0.0202 \\
-\end{array}$ & $\begin{array}{c}0.0036 \\
0.0005 \\
0.0034 \\
-\end{array}$ & $\begin{array}{c}26 \\
12 \\
11 \\
3\end{array}$ & $\begin{array}{l}18 \\
11 \\
4 \\
3\end{array}$ \\
\hline $\begin{array}{l}\text { Lognormal } \\
\hat{\mu}_{2}\end{array}$ & One-sided & $\begin{array}{l}\text { Combined } \\
\text { Glass } \\
\text { Metal } \\
\text { Poly }\end{array}$ & $\begin{array}{c}0.0131 \\
0.0134 \\
0.0188 \\
-\end{array}$ & $\begin{array}{c}0.0019 \\
0.0005 \\
0.0026 \\
-\end{array}$ & $\begin{array}{l}26 \\
12 \\
11 \\
3\end{array}$ & $\begin{array}{c}18 \\
11 \\
4 \\
3\end{array}$ \\
\hline $\begin{array}{l}\text { Normal } \\
\hat{\mu}_{3}\end{array}$ & Interval & $\begin{array}{l}\text { Combined } \\
\text { Glass } \\
\text { Metal } \\
\text { Poly }\end{array}$ & $\begin{array}{l}0.0191 \\
0.0134 \\
0.0233 \\
0.0322\end{array}$ & $\begin{array}{l}0.0022 \\
0.0005 \\
0.0028 \\
0.0098\end{array}$ & $\begin{array}{l}26 \\
12 \\
11 \\
3\end{array}$ & $\begin{array}{l}18 \\
11 \\
4 \\
3\end{array}$ \\
\hline $\begin{array}{l}\text { Lognormal } \\
\hat{\mu}_{A}\end{array}$ & Interval & $\begin{array}{l}\text { Combined } \\
\text { Glass } \\
\text { Metal } \\
\text { Poly }\end{array}$ & $\begin{array}{l}0.0169 \\
0.0134 \\
0.0215 \\
0.0281\end{array}$ & $\begin{array}{l}0.0017 \\
0.0005 \\
0.0025 \\
0.0085\end{array}$ & $\begin{array}{l}26 \\
12 \\
11 \\
3\end{array}$ & $\begin{array}{l}18 \\
11 \\
4 \\
3\end{array}$ \\
\hline $\begin{array}{l}\text { Kaplan-Meier } \\
\hat{\mu}_{5}\end{array}$ & One-sided & $\begin{array}{l}\text { Combined } \\
\text { Glass } \\
\text { Metal } \\
\text { Poly }\end{array}$ & $\begin{array}{c}0.0158 \\
0.0140 \\
0.0204 \\
-\end{array}$ & $\begin{array}{c}0.0016 \\
- \\
0.0032 \\
-\end{array}$ & $\begin{array}{l}26 \\
12 \\
11 \\
3\end{array}$ & $\begin{array}{l}18 \\
11 \\
4 \\
3\end{array}$ \\
\hline $\begin{array}{l}\text { Normal Sample Mean } \\
\text { (Upper bound) } \\
\bar{\mu}_{6}\end{array}$ & Ignored & $\begin{array}{l}\text { Combined } \\
\text { Glass } \\
\text { Metal } \\
\text { Poly }\end{array}$ & $\begin{array}{l}0.0225 \\
0.0164 \\
0.0252 \\
0.0366\end{array}$ & $\begin{array}{l}0.0022 \\
0.0006 \\
0.0032 \\
0.0115\end{array}$ & $\begin{array}{l}26 \\
12 \\
11 \\
3\end{array}$ & $\begin{array}{c}18 \\
11 \\
4 \\
3\end{array}$ \\
\hline $\begin{array}{l}\text { Normal Sample Mean } \\
\text { (Lower bound) } \\
\underline{\mu}_{6}\end{array}$ & Set to 0 & $\begin{array}{l}\text { Combined } \\
\text { Glass } \\
\text { Metal } \\
\text { Poly }\end{array}$ & $\begin{array}{l}0.0156 \\
0.0072 \\
0.0216 \\
0.0276\end{array}$ & $\begin{array}{l}0.0023 \\
0.0008 \\
0.0029 \\
0.0123\end{array}$ & $\begin{array}{l}26 \\
12 \\
11 \\
3\end{array}$ & $\begin{array}{l}18 \\
11 \\
4 \\
3\end{array}$ \\
\hline $\begin{array}{l}\text { Lognormal Sample Mean } \\
\text { (Upper bound) } \\
\bar{\mu}_{7}\end{array}$ & Ignored & $\begin{array}{l}\text { Combined } \\
\text { Glass } \\
\text { Metal } \\
\text { Poly }\end{array}$ & $\begin{array}{l}0.0206 \\
0.0163 \\
0.0232 \\
0.0333\end{array}$ & $\begin{array}{c}0.0016 \\
0.0006 \\
0.0030 \\
0.100\end{array}$ & $\begin{array}{l}26 \\
12 \\
11 \\
3\end{array}$ & $\begin{array}{l}18 \\
11 \\
4 \\
3\end{array}$ \\
\hline $\begin{array}{l}\text { Lognormal Sample Mean } \\
\text { (Lower bound) } \\
\underline{\mu}_{7}\end{array}$ & Set to 0 & $\begin{array}{l}\text { Combined } \\
\text { Glass } \\
\text { Metal } \\
\text { Poly }\end{array}$ & $\begin{array}{l}0.0123 \\
0.0068 \\
0.0200 \\
0.0227\end{array}$ & $\begin{array}{l}0.0017 \\
0.0007 \\
0.0024 \\
0.0098\end{array}$ & $\begin{array}{c}26 \\
12 \\
11 \\
3\end{array}$ & $\begin{array}{c}18 \\
11 \\
4 \\
3\end{array}$ \\
\hline
\end{tabular}


TABLE S(a). ESTMMATION OF TRU PROPORTON FROM BOTH SAMPLE SETS NO OBSERVATIONS OMITIED

\begin{tabular}{|c|c|c|c|c|c|c|}
\hline Method & Type Cens & Waste Comp & Estimate & Std Ers & $n$ & \#Cens \\
\hline $\begin{array}{l}\overline{\text { Normal }} \\
\hat{\mu}_{1}\end{array}$ & One-sided & $\begin{array}{l}\text { Combined } \\
\text { Cell } \\
\text { Glass } \\
\text { Metal } \\
\text { Poly } \\
\text { Wipes }\end{array}$ & $\begin{array}{c}0.0079 \\
-0.0005 \\
0.0133 \\
0.0199 \\
0.0032 \\
0.0220\end{array}$ & $\begin{array}{l}0.0045 \\
0.0215 \\
0.0005 \\
0.0033 \\
0.0151 \\
0.0141\end{array}$ & $\begin{array}{c}62 \\
9 \\
18 \\
16 \\
14 \\
5\end{array}$ & $\begin{array}{c}50 \\
8 \\
17 \\
9 \\
13 \\
3\end{array}$ \\
\hline $\begin{array}{l}\text { Lognormal } \\
\hat{A}_{2}\end{array}$ & One-sided & $\begin{array}{l}\text { Combined } \\
\text { Cell } \\
\text { Glass } \\
\text { Metal } \\
\text { Poly } \\
\text { Wipes }\end{array}$ & $\begin{array}{l}0.0116 \\
0.0072 \\
0.0134 \\
0.0186 \\
0.0061 \\
0.0240\end{array}$ & $\begin{array}{l}0.0020 \\
0.0072 \\
0.0005 \\
0.0025 \\
0.0057 \\
0.0085\end{array}$ & $\begin{array}{c}62 \\
9 \\
18 \\
16 \\
14 \\
5\end{array}$ & $\begin{array}{c}50 \\
8 \\
17 \\
9 \\
13 \\
3\end{array}$ \\
\hline $\begin{array}{l}\text { Normal } \\
\hat{\mu}_{3}\end{array}$ & Interval & $\begin{array}{l}\text { Combined } \\
\text { Cell } \\
\text { Glass . } \\
\text { Metal } \\
\text { Poly } \\
\text { Wipes }\end{array}$ & $\begin{array}{l}0.0285 \\
0.0331 \\
0.0259 \\
0.0268 \\
0.0298 \\
0.0313\end{array}$ & $\begin{array}{l}0.0029 \\
0.0132 \\
0.0064 \\
0.0025 \\
0.0035 \\
0.0061\end{array}$ & $\begin{array}{c}62 \\
9 \\
18 \\
16 \\
14 \\
5\end{array}$ & $\begin{array}{c}50 \\
8 \\
17 \\
9 \\
13 \\
3\end{array}$ \\
\hline $\begin{array}{l}\text { Lognormal } \\
A_{4}\end{array}$ & Interval & $\begin{array}{l}\text { Combined } \\
\text { Cell } \\
\text { Gless } \\
\text { Metal } \\
\text { Poly } \\
\text { Wipes }\end{array}$ & $\begin{array}{l}0.0230 \\
0.0218 \\
0.0176 \\
0.0248 \\
0.0264 \\
0.0289\end{array}$ & $\begin{array}{l}0.0019 \\
0.0062 \\
0.0034 \\
0.0025 \\
0.0037 \\
0.0051\end{array}$ & $\begin{array}{c}62 \\
9 \\
18 \\
16 \\
14 \\
5\end{array}$ & $\begin{array}{c}50 \\
8 \\
17 \\
9 \\
13 \\
3\end{array}$ \\
\hline $\begin{array}{l}\text { Kaplan-Meier } \\
\boldsymbol{\rho}_{5}\end{array}$ & One-sided & $\begin{array}{l}\text { Combined } \\
\text { Cell } \\
\text { Glass } \\
\text { Metal } \\
\text { Poly } \\
\text { Wipes }\end{array}$ & $\begin{array}{l}0.0165 \\
0.0294 \\
0.0140 \\
0.0200 \\
0.0257 \\
0.0348\end{array}$ & $\begin{array}{c}0.0013 \\
\cdot \\
0.0030 \\
0.0069\end{array}$ & $\begin{array}{c}62 \\
9 \\
18 \\
16 \\
14 \\
5\end{array}$ & $\begin{array}{c}50 \\
8 \\
17 \\
9 \\
13 \\
3\end{array}$ \\
\hline $\begin{array}{l}\text { Normal Sample Mean } \\
\text { (Upper limit) } \\
\bar{\mu}_{6}\end{array}$ & Ignored & $\begin{array}{l}\text { Combined } \\
\text { Cell } \\
\text { Glass } \\
\text { Metal } \\
\text { Poly } \\
\text { Wipes }\end{array}$ & $\begin{array}{l}0.0325 \\
0.0374 \\
0.0307 \\
0.0299 \\
0.0339 \\
0.0343\end{array}$ & $\begin{array}{l}0.0030 \\
0.0141 \\
0.0066 \\
0.0029 \\
0.0039 \\
0.0058\end{array}$ & $\begin{array}{c}62 \\
9 \\
18 \\
16 \\
14 \\
5\end{array}$ & $\begin{array}{c}50 \\
8 \\
17 \\
9 \\
13 \\
3\end{array}$ \\
\hline $\begin{array}{l}\text { Normal Sarnple Mean } \\
\text { (Lower bound) } \\
\text { Ho }_{6}\end{array}$ & Set to 0 & $\begin{array}{l}\text { Combined } \\
\text { Cell } \\
\text { Glass } \\
\text { Metel } \\
\text { Poly } \\
\text { Wipes }\end{array}$ & $\begin{array}{l}0.0247 \\
0.0289 \\
0.0211 \\
0.0241 \\
0.0260 \\
0.0278\end{array}$ & $\begin{array}{l}0.0029 \\
0.0140 \\
0.0065 \\
0.0023 \\
0.0036 \\
0.0076\end{array}$ & $\begin{array}{l}62 \\
9 \\
18 \\
16 \\
14 \\
5\end{array}$ & $\begin{array}{c}50 \\
8 \\
17 \\
9 \\
13 \\
3\end{array}$ \\
\hline $\begin{array}{l}\text { Lognormal Sample Mean } \\
\text { (Upper bound) } \\
\bar{\mu}_{7}\end{array}$ & Ignored & $\begin{array}{l}\text { Combined } \\
\text { Cell } \\
\text { Glass } \\
\text { Metal } \\
\text { Poly } \\
\text { Wipes }\end{array}$ & $\begin{array}{l}0.0272 \\
0.0272 \\
0.0237 \\
0.0275 \\
0.0302 \\
0.0327\end{array}$ & $\begin{array}{l}0.0020 \\
0.0067 \\
0.0037 \\
0.0030 \\
0.0045 \\
0.0048\end{array}$ & $\begin{array}{c}62 \\
9 \\
18 \\
16 \\
14 \\
5\end{array}$ & $\begin{array}{c}50 \\
8 \\
17 \\
9 \\
13 \\
3\end{array}$ \\
\hline $\begin{array}{l}\text { Lognomal Sample Mean } \\
\text { (Lower bound) } \\
\underline{\mu}_{7}\end{array}$ & Set to 0 & $\begin{array}{l}\text { Combined } \\
\text { Cell } \\
\text { Glass } \\
\text { Metal } \\
\text { Poly } \\
\text { Wipes }\end{array}$ & $\begin{array}{l}0.0177 \\
0.0144 \\
0.0119 \\
0.0225 \\
0.0230 \\
0.0245\end{array}$ & $\begin{array}{l}0.0019 \\
0.0064 \\
0.0028 \\
0.0022 \\
0.0033 \\
0.0059\end{array}$ & $\begin{array}{c}62 \\
9 \\
18 \\
16 \\
14 \\
5\end{array}$ & $\begin{array}{c}50 \\
8 \\
17 \\
9 \\
13 \\
3\end{array}$ \\
\hline
\end{tabular}


TABLE S(b). ESTTMATION OF TRU PROPORTION FROM BOTH SAMILE SETS FOUR OUTLIFR OBSERVATIONS OMITIED

\begin{tabular}{|c|c|c|c|c|c|c|}
\hline Mabrod & Type Cens & Warte Comp & Estimnite & Std ET & $n$ & *Cens \\
\hline $\begin{array}{l}\text { Normal } \\
a_{1}\end{array}$ & Onc-sided & $\begin{array}{l}\text { Combined } \\
\text { Cell } \\
\text { Glass } \\
\text { Metal } \\
\text { Poly } \\
\text { Wipes }\end{array}$ & $\begin{array}{l}0.0089 \\
0.0066 \\
0.0134 \\
0.0199 \\
0.0032 \\
0.00220\end{array}$ & $\begin{array}{l}0.0044 \\
0.0164 \\
0.0005 \\
0.0033 \\
0.0151 \\
0.0141\end{array}$ & $\begin{array}{l}58 \\
7 \\
17 \\
15 \\
14 \\
5\end{array}$ & $\begin{array}{c}46 \\
6 \\
16 \\
8 \\
13 \\
3\end{array}$ \\
\hline $\begin{array}{l}\text { Lognormal } \\
A_{2}\end{array}$ & One-sided & $\begin{array}{l}\text { Combined } \\
\text { Cell } \\
\text { Glass } \\
\text { Mets } \\
\text { Poly } \\
\text { Wipes }\end{array}$ & $\begin{array}{l}0.0121 \\
0.0110 \\
0.0134 \\
0.0186 \\
0.0061 \\
0.0240\end{array}$ & $\begin{array}{l}0.0020 \\
0.0078 \\
0.0005 \\
0.0025 \\
0.0057 \\
0.0085\end{array}$ & $\begin{array}{l}58 \\
7 \\
17 \\
15 \\
14 \\
5\end{array}$ & $\begin{array}{c}46 \\
6 \\
16 \\
8 \\
13 \\
3\end{array}$ \\
\hline $\begin{array}{l}\text { Normal } \\
\hat{\mu}_{3} \\
{ }_{1}\end{array}$ & Interval & $\begin{array}{l}\text { Combined } \\
\text { Cell } \\
\text { Gless } \\
\text { Metal } \\
\text { Poly } \\
\text { Wipes }\end{array}$ & $\begin{array}{l}0.0270 \\
0.0214 \\
0.0265 \\
0.0261 \\
0.0298 \\
0.0313\end{array}$ & $\begin{array}{l}0.0024 \\
0.0029 \\
0.0067 \\
0.0025 \\
0.0035 \\
0.0061\end{array}$ & $\begin{array}{c}58 \\
7 \\
17 \\
15 \\
14 \\
5\end{array}$ & $\begin{array}{c}46 \\
6 \\
16 \\
8 \\
13 \\
3\end{array}$ \\
\hline $\begin{array}{l}\text { Lognormal } \\
A_{4}\end{array}$ & Interval & $\begin{array}{l}\text { Combined } \\
\text { Cell } \\
\text { Glass } \\
\text { Metal } \\
\text { Poly } \\
\text { Wipes }\end{array}$ & $\begin{array}{l}0.0227 \\
0.0202 \\
0.0177 \\
0.0242 \\
0.0264 \\
0.0289\end{array}$ & $\begin{array}{l}0.0018 \\
0.0029 \\
0.0036 \\
0.0025 \\
0.0037 \\
0.0051\end{array}$ & $\begin{array}{l}58 \\
7 \\
17 \\
15 \\
14 \\
5\end{array}$ & $\begin{array}{c}46 \\
6 \\
16 \\
8 \\
13 \\
3\end{array}$ \\
\hline $\begin{array}{l}\text { Keplan-Meier } \\
\boldsymbol{\mu}_{5}\end{array}$ & One-sided & $\begin{array}{l}\text { Combined } \\
\text { Cell } \\
\text { Glass } \\
\text { Metal } \\
\text { Poly } \\
\text { Wipes }\end{array}$ & $\begin{array}{l}0.0168 \\
0.0294 \\
0.0140 \\
0.0200 \\
0.0257 \\
0.0348\end{array}$ & $\begin{array}{c}0.0014 \\
. \\
0.0030 \\
0.0069\end{array}$ & $\begin{array}{l}58 \\
7 \\
17 \\
15 \\
14 \\
5\end{array}$ & $\begin{array}{c}46 \\
6 \\
16 \\
8 \\
13 \\
3\end{array}$ \\
\hline $\begin{array}{l}\text { Normal Sample Mean } \\
\text { (Upper bound) } \\
\bar{\mu}_{6}\end{array}$ & Ignored & $\begin{array}{l}\text { Combined } \\
\text { Cell } \\
\text { Gloss } \\
\text { Metel } \\
\text { Poly } \\
\text { Wipes }\end{array}$ & $\begin{array}{l}0.0309 \\
0.0252 \\
0.0313 \\
0.0290 \\
0.0339 \\
0.0343\end{array}$ & $\begin{array}{l}0.0024 \\
0.0033 \\
0.0070 \\
0.0029 \\
0.0039 \\
0.0058\end{array}$ & $\begin{array}{l}58 \\
7 \\
17 \\
15 \\
14 \\
5\end{array}$ & $\begin{array}{c}46 \\
6 \\
16 \\
8 \\
13 \\
3\end{array}$ \\
\hline $\begin{array}{l}\text { Normal Sample Mean } \\
\text { (Lower bound) } \\
\text { ut }_{6}\end{array}$ & Set to 0 & $\begin{array}{l}\text { Combined } \\
\text { Cell } \\
\text { Glaes } \\
\text { Metal } \\
\text { Poly } \\
\text { Wipes }\end{array}$ & $\begin{array}{l}0.0232 \\
0.0174 \\
0.0217 \\
0.0236 \\
0.0260 \\
0.0278\end{array}$ & $\begin{array}{l}0.0024 \\
0.0035 \\
0.0069 \\
0.0024 \\
0.0034 \\
0.0076\end{array}$ & $\begin{array}{l}58 \\
7 \\
17 \\
15 \\
14 \\
5\end{array}$ & $\begin{array}{c}46 \\
6 \\
16 \\
8 \\
13 \\
3\end{array}$ \\
\hline $\begin{array}{l}\text { Lognormal Sample Mean } \\
\text { (Upper bound) } \\
\bar{\mu}_{7}\end{array}$ & Ignored & $\begin{array}{l}\text { Combined } \\
\text { Cell } \\
\text { Glass } \\
\text { Metal } \\
\text { Poly } \\
\text { Wipes }\end{array}$ & $\begin{array}{l}0.0267 \\
0.0240 \\
0.0239 \\
0.0267 \\
0.0302 \\
0.0327\end{array}$ & $\begin{array}{l}0.0019 \\
0.0030 \\
0.0039 \\
0.0030 \\
0.0045 \\
0.0048\end{array}$ & $\begin{array}{c}58 \\
7 \\
17 \\
15 \\
14 \\
5\end{array}$ & $\begin{array}{c}46 \\
6 \\
16 \\
8 \\
13 \\
3\end{array}$ \\
\hline $\begin{array}{l}\text { Lognormal Sample Mean } \\
\text { (Lower bound) } \\
\mu_{7}\end{array}$ & Set to 0 & $\begin{array}{l}\text { Combined } \\
\text { Cell } \\
\text { Glass } \\
\text { Metal } \\
\text { Poly } \\
\text { Wipes }\end{array}$ & $\begin{array}{l}0.0180 \\
0.0152 \\
0.0120 \\
0.0220 \\
0.0230 \\
0.0245\end{array}$ & $\begin{array}{l}0.0017 \\
0.0032 \\
0.0030 \\
0.0022 \\
0.0033 \\
0.0059\end{array}$ & $\begin{array}{c}58 \\
7 \\
17 \\
15 \\
14 \\
5\end{array}$ & $\begin{array}{c}46 \\
6 \\
16 \\
8 \\
13 \\
3\end{array}$ \\
\hline
\end{tabular}


the number of samples (or observations); and the number of censored (or nondetect) observations. Taking into account that the logarithmic transform makes the distribution of the observations closer to the normal distribution, we recommend the use of $\hat{\mu}_{4}$ for further studies to estimate $\mu$ and to control (validate) the results with $\underline{\mu}_{7}$ and $\bar{\mu}_{7}$. The most obvious conclusion from a review of Tables $3-5$ is that the estimates of the mean proportion TRU are systematically less in sample set 2 than in sample set 1 . The estimates (Table 5) based on the combined information from both data sets neglect this fact and are recommended to be used only at preliminary stages of similar studies, for instance, when the sample sizes are relatively small ( 15 - 20 cases). Note, that the gross alpha activity was generally lower for set 1 than for set 2, leading to distinctly different estimates of PTRU. Thus, whenever there exists a possibility to stratify observations in accordance with the observed gross alpha activity, which is easy to measure, we recommend doing this stratification. The analysis of residuals from the estimates reported above leads to the recommendation of the estimate $\hat{\mu}_{4}$, which is the maximum likelihood estimate for the interval censored observations constructed under the assumption that the logarithms of observed values are normally distributed. The simultaneous use of estimates $\underline{\mu}_{7}$ and $\bar{\mu}_{7}$ gives an opportunity to evaluate the uncertainty imposed by the presence of nondetects. Their computation is strongly recommended when the fraction of nondetects is high, say greater than $10 \%$.

\subsection{Regression analysis}

To analyze the tendency of the proportion TRU to decrease when the gross alpha activity increases, we regressed the proportion TRU (PTRU) activity on the gross alpha activity (GA) (both log transformed) for both sample sets individually and combined. The linear regression model for this is

$$
y_{\alpha i}=\beta_{\alpha 0}+\beta_{\alpha 1} x_{\alpha i}+\varepsilon_{\alpha i}
$$

and the quadratic regression model is given by

$$
y_{\alpha i}=\beta_{\alpha 0}+\beta_{\alpha 1} x_{\alpha i}+\beta_{\alpha 2} x_{\alpha i}^{2}+\varepsilon_{\alpha i},
$$


where $y_{\alpha i}=\ln \frac{T R U_{a i}}{G A_{a i}}$ may be considered as hypothetical observations with all nondetects replaced by actual (unfortunately frequently unknown) values, $x_{\alpha i}=\ln G A_{\alpha i}, G A_{\alpha i}$ is the mean gross alpha activity, for sample $i$ (each sample was measured twice for gross alpha activity), $\alpha$ indicates the sample set number, $\beta$ 's are model parameters to be estimated; and $\varepsilon_{\alpha i}$ is the random noise component for observation $y_{\alpha i}$ with mean $0\left(i=1,2, \ldots, n_{\alpha}\right.$ and $\left.\alpha=1,2\right)$. We did not construct different regression models for glass, metal, etc., for two reasons. First, the previous analysis did not reveal any statistically significant difference between TRU or PTRU for various materials. Second, we do not have the luxury to partition the data sets into smaller pieces because of the limited sample size, large number of nondetects within some waste types, and the additional parameters to be estimated.

Of course, both (10) and (11) are alternatives to the previously mentioned model (4) in Section 4, which in the current notation may be presented as

$$
y_{\alpha i}=\beta_{\alpha 0}+\varepsilon_{\alpha i}, \beta_{\alpha 0}=\ln f
$$

Table 6 contains the regression results for the linear and quadratic models. Because not all $y_{\alpha i}$ are known, we performed analysis using the lower and upper bounds for TRU (compare with (5) and (6) of Section 5.1). Correspondingly, Table 6 contains two sets of parameter estimates for every considered case, one for the linear and one for the quadratic model. Similar to (8) of Section 5.1, we may assert that

$$
\underline{\beta}_{\alpha 0}+\underline{\beta}_{\alpha 1} x_{\alpha i} \leq \hat{\beta}_{\alpha 0}+\hat{\beta}_{\alpha 1} x_{\alpha i} \leq \bar{\beta}_{\alpha 0}+\bar{\beta}_{\alpha 1} x_{\alpha i}
$$

for all $x_{\alpha i}$. In the above expression, the underlined letters correspond to parameter estimates based on the lower bound observations and the letters with upper bars to the upper bound; compare with (7). The extension of (13) for the quadratic case is obvious.

What can be concluded from Table 6? Let us start with the quadratic case. The "rule of thumb", assuming normality, for determining significance of the parameters is that the ratio of the parameter's estimate (absolute value) to its standard error is 
Table 6: Parameter Estimates from Regression of n (Proportion TRU) on n (Gross Alpha)

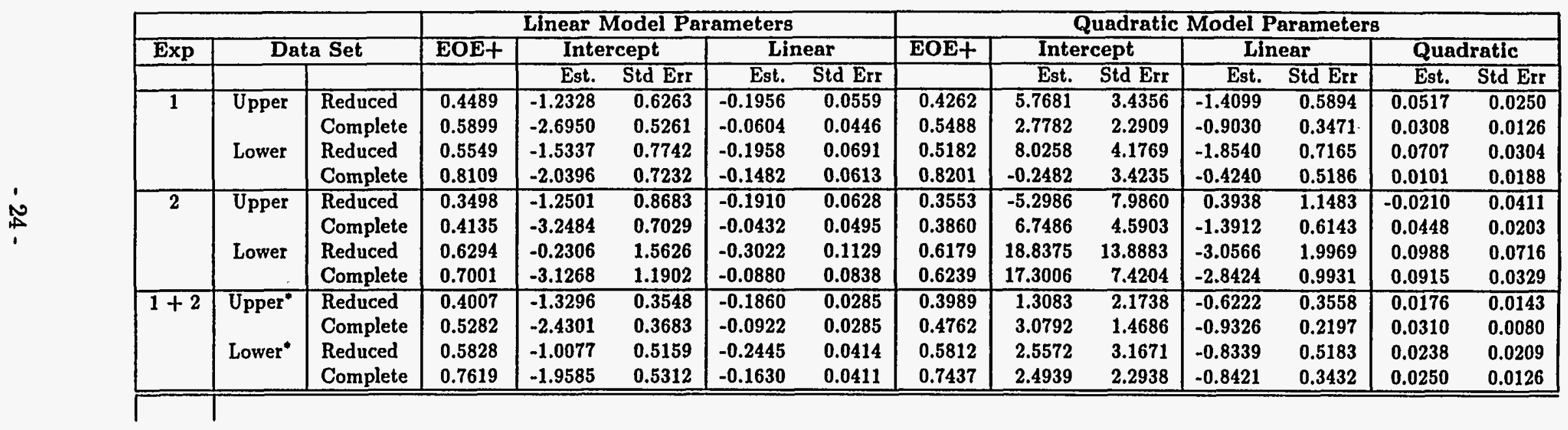

- See page 26 .

$+\mathrm{EOE}=$ estimated observational error = square root of mean square error. 
greater than 2 (in this case, the probability we will reject a parameter whose true value is zero is approximately 0.05). More than half of the cases in the last column of Table 6 satisfy this rule, implying that the quadratic coefficient is significant. However, we are very reluctant to recommend the use of quadratic models in the future for the reasons given in the following two paragraphs.

First, the presence of nondetects and the subsequent use of "upper bounds" and "lower bounds" makes the assumption about normality theoretically unacceptable, even for a relatively large number of observations, a case when normality is considered frequently as a corollary of the Central Limit Theorem (see, for instance, [19]). For a smaller number of observations, the assumption about normality of the underlying distribution is even less tenable because other types of distributions correspond better with our situation (see Appendix 3).

Second, the estimates of the quadratic coefficients are sensitive to the deleting of "leverage points", which were mentioned in Section 4 (see the last column of Table 6). The nonstability of the estimates indicates that the significance of the corresponding parameters may be spurious and induced by unknown factors, which have caused those two points at each of the sample sets to be far away from the main data clouds.

The linear model (10) is obviously a more reliable choice, and we recommend its use, but with some reservations: the practitioner has to remember that the standard regression analysis theory is not applicable to the full extent and the above remarks (for instance, about normality) are still valid here.

From all the linear models included in Table 6, it follows that the proportion of TRU is decreasing as the level of gross alpha activity increases. This tendency, being statistically significant, does not however change the proportion TRU too much. For instance, for the data sets with deleted outliers the slopes are in the range $[-0.30,-0.19]$ in log-log space. Figures 7 - 10 give a visual presentation of the regression models, which were discussed above. For instance, one can see that the change in the logarithm of the proportion of TRU per unit change in the log scale of the gross alpha (i.e., the slope of the regression lines) is several times less than the estimated observational error (EOE). Actually, the corresponding ratios of the EOE to the estimated slope vary from 4.7 to 
9.8; see Table 6, linear model results. We did not test the homogeneity of variance of the transformed observations because the number of observations was not sufficient for any statistically reliable inference. All the results were derived under the assumption that this variance is constant. It is well known that the introduction of weights to address non-homogeneity do not change the parameter estimates significantly and mainly lead to more realistic inferences about their standard errors.

We recommend use of the results of the regression analysis with the removed outliers. It is especially important for the second data set, in which both outliers correspond to leached items rather than smears (they are marked in Table A2 as LN-GL and LN-ML). The observed proportion TRU is in excellent agreement with the matching smears (LN-1, LN-11 and LN-7, LN-15 correspondingly) and can be used in constructing mean proportion TRU (i.e., estimates reported in Tables 3-4). However, the gross alpha readings for those two cases are not compatible with the corresponding readings for smears. Therefore, it can lead to a significant distortion of the estimate of the slope. This fact can be easily verified with the help of Fig. $8 \mathrm{a}$ and $8 \mathrm{~b}$. Following the above discussion, we recommend the linear models which are marked by asterisks in Table 6 and also presented in Fig. 9 (c and d) and Fig. 10 ( $a$ and b). In this table "reduced" refers to data sets with outlier observations deleted, i.e., two from set 1 and two from set 2.

\subsection{Application to NDA}

In order to apply the regression models to NDA, it is necessary that we convert $x=$ "gross alpha activities" to $Z=$ "total concentration for alpha emitters". This conversion can be made with the help of the proper calibration experiments. However, we have only one sample (LN-GL) where both variables, $X$ and $Z$, are available which are matched with IN-1 and LN-11. Table A3 summarizes the results from the leaching sample LN-GL. In particular $Z \cong 4.0 \times 10^{4} n C i / g$ which corresponds to the mean activity from two smears, which equals $1.1 \times 10^{6} B q$. From these results, the conversion factor can be roughly estimated as $K=25.2$. Bounds on this factor may be obtained by using bounds on the estimated low and high end weights. Replacing $x$ by $K \times Z$, 
i.e., using the model

$$
\text { In }(P T \hat{R} U)=\hat{\beta}_{0}+\hat{\beta}_{1} \ln (K \times Z)
$$

where the parameters $\hat{\beta}_{0}$ and $\hat{\beta}_{1}$ come from Table 6 , we can estimate the proportion TRU knowing the total concentration for alpha emitters. The uncertainty of this equation can be partially evaluated by using the above bounds on $K$.

In order to make the conversion results statistically valid, we must have results from additional leaching experiments matched with smears. Having the corresponding results, one can construct a better estimate for the conversion factor. Of course, when the number of pairs "leaching - smearing" is large enough (say 10-15) then direct construction of model PTRU $=F(Z)$ is possible. However, the cost of leaching is substantially higher than the cost of the smear analysis. In particular, this fact forced us to proceed in this work with the samples based on smearing. Separate conversions must be determined for each waste type (glass, metal, etc.) because of the different densities across waste types.

\subsection{Comments on proportion TRU trend}

Discussions with REDC operations personnel revealed that the trend found above may be valid from a physical point of view. One of the possible explanations is the following one.

The REDC processing campaign aims to not only separate the actinides from fission products, but to also partition the actinides from each other. The targets are made of predominantly $\mathrm{Cm}$ and are still predominantly $\mathrm{Cm}$ after irradiation in the HFIR. The calculations on the HFIR targets showed that, as of $3 / 5 / 96$, the targets contained a total of approximately 0.13 grams of $\mathrm{Pu}(0.32 \mathrm{Ci})$ and 0.08 grams of Am (0.016 $\mathrm{Ci})$ as compared to the 64.6 grams of $\mathrm{Cm}(\sim 2300 \mathrm{Ci})$. This relatively large mass of $\mathrm{Cm}$ is the reason that all of the waste coming from the REDC cell bank is heavily contaminated with Cm244. One of the first steps in the process is to remove the rare earth fission products from the actinide products. Once this has been sufficiently accomplished, the actinides themselves are separated from each other. At this point in the process, there are different streams going into their respective tanks for further 
processing. For example, the $\mathrm{Cm}$ product (most abundant and highest activity) will be relatively free of $\mathrm{Pu}, \mathrm{Am}, \mathrm{Cf}, \mathrm{Es}$, etc. after this initial processing. Any materials coming into contact with this $\mathrm{Cm}$ product (sample bottles, transfer lines, etc.) will be predominately contaminated with $\mathrm{Cm}$, as there are only trace levels of other actinides as impurities in the product. Noting the total activities of $\mathrm{Am}, \mathrm{Pu}$ and $\mathrm{Cm}$ at target discharge, if the product volumes are equal, then waste from the $\mathrm{Cm}$ stream will be much higher in total activity than waste from the $\mathrm{Pu}$ stream. Since this work has shown $\mathrm{Am}$ and $\mathrm{Pu}$ to be the greatest contributors to the TRU activity, it is not surprising that their relative percentages decrease when the activity (and hence $\mathrm{Cm}$ ) increases. On the other hand, materials that have been in contact with the other product streams ( $\mathrm{Am}, \mathrm{Pu}$ ) would be expected not to be as high in total activity, yet have a higher TRU percentage than a $\mathrm{Cm}$ product stream.

\section{Summary}

The primary objective of this work was to obtain an understanding and a proper statistical description of the elemental and isotopic structure of solid waste generated at REDC, for the ultimate purpose of classifying waste as TRU or not TRU. This was achieved through an investigation of the isotopic profile of alpha-emitting radionuclides. Prior to this study, we anticipated some stability in the isotopic profiles for various waste types or items. Our goal was to verify this and to estimate the corresponding profile, by measuring smears and leached samples. All of the statistical conclusions and inferences along with associated recommendations presented in this report are based on the assumed representativeness of the available data used.

Models and methods. Before analyzing the data, the main sources of uncertainty contributing to the variability in the observed data were identified and they included:

i. variability from one campaign to another (in this study,we were restricted to only Campaign 69);

ii. variability across different items or materials (e.g., plastic, glass, etc);

iii. variability in the selection of the item to be sampled or measured;

iv. randomness associated with the smearing process; and 
v. randomness in the observation error for the various analytical techniques.

The choice of the appropriate underlying stochastic models for the observed activities were evaluated for a number of intuitively appealing sets of physical assumptions. All of them were proven to lead to the same type estimator of the isotopic proportion. The form of the statistically optimal estimator was specified as a ratio of means rather than a mean of ratios, which was used in this study because of the form the original data were provided. The derived maximum likelihood estimators provide efficient estimation of the proportion TRU and are recommended for future work. The results of the estimation problem using different physical assumptions are summarized in Appendix 3.

To address properly the randomness of the observational errors in analytical measurements, we analyzed the Poisson regression model in Appendix 2 and showed that the currently-used method of estimating individual isotopic activities is a simplified version of the exact, and statistically more efficient, maximum likelihood estimator. The latter leads to iterative regression methods and the corresponding numerical algorithms which are reported in Appendix 2 are recommended for future work.

Experimental results and analysis. The available data sets and, in particular, the data from set 1 , contain a large number of cases where at least one of the TRU components was below the detection level, primarily CM246. The second sample achieved a higher resolution for the plutonium isotopes through chemical separation. The presence of the "less than" observations (or nondetects) necessitated the development of an approach, which includes several different statistical estimators, to find statistically reliable estimates of the proportion TRU (see Sections 4 and 5).

If the data from the two sample sets used in this study can be assumed to be representative samples for the campaign under analysis, then the results of the statistical analysis have shown the following:

i. No statistically significant difference in the proportion TRU across the different waste types (glass, metal, etc.) was detected, i.e., the variability from one waste type to another was comparable to the sampling variability within a given waste type. However, minimal data were available to make possible the statistical comparison for some of the 
waste types.

ii. The hypothesis about the stability of the proportion TRU as a function of the sample gross alpha activity was rejected. Using regression analysis, a linear trend was revealed that showed the log proportion TRU to decrease as the log gross alpha activity increased. The reader should remember that statistical significance does not coincide generally with practical importance (see Section 5.2).

iii. The estimated mean proportion TRU ranged approximately from 0.01 to 0.05 within the range of the gross alpha activity which characterized the waste in the two sample sets.

iv. Cm244 was the dominant alpha-emitting radionuclide with more than $93 \%$ of the samples from the combined sample sets having a proportion Cm244 greater than 0.90 .

v. Three choices of the detection limits for the plutonium isotopes $(0.05 \%, 0.5 \%$ and $1 \%$ of gross alpha) did not reveal any practical discrepancy in the results, i.e., all conclusions remained unaffected. A graphical confirmation of that fact can be found from an examination and comparison of Figs. 10b, c, and $d$.

Although the trend in the proportion TRU as a function of the gross alpha activity mentioned above was based on the sample activity measurements from smear samples, when this result is used in the NDA, additional calibration of this dependency is needed using direct concentration rather than activity measurements. These concentration measurements are available only through leaching techniques, which were used with only one sample unit in this work. Hence, our recommendation is that at least several $(>4)$ leaching tests be run for every type of material preferably around the official 100 $\mathrm{nCi} / \mathrm{g}$ threshold used for deciding whether the waste under consideration is TRU or not.

Non TRU radionuclide. The software and methodology developed in this work may be used for the analysis of any radionuclide listed in Tables A1 and A2, including beta-gamma emitters. Of course, we have to be very cautious with any formal statistical inferences when the number of nondetects is extremely high. Analysis and results for Cf252 have been performed for this project and are available upon request. They 
are not reported in this publication because the results would double the size of this report. We note that for $\mathrm{Cf} 252$ all tendencies are very similar to those found for TRU. For instance, the proportion Cf252 decreases with increase of the total alpha activity, and this dependence is even more pronounced than in the TRU case. For beta-gamma emitters, the analysis of their correlation with TRU radionuclides can help in NDA. The corresponding results will be reported in [21].

Perspectives to complement NDA procedures. In spite of the presence of the above-mentioned linear trend, we can make statements about the average proportion TRU which may be applied to the entire range of sampled activity assuming a realistic censored underlying distribution for the observed proportions. From both the regression analysis and the estimates obtained from the censored distribution analysis, we conclude that the proportion TRU ranges from approximately $0.005-0.06$. Under rather restrictive assumptions (e.g., log normality of the observations), this interval may be considered as a conservative bound on the $95 \%$ confidence interval for the mean proportion TRU, i.e., the actual confidence interval may fall within this interval. If additional leaching tests are performed, then the length of the confidence interval would be reduced by taking the linear trend into account. The previously mentioned interval will be replaced by 2 to 3 times the smaller interval: $P T R U \pm 0.01$. The latter interval is a very simplistic approximation of what can be derived from the regression models constructed in this work after proper calibration in the $\mathrm{nCi} / \mathrm{g}$ scale. This interval is crucial for the current NDA technology which does not allow for the reliable separation of activities for individual isotopes dominated by $\mathrm{Cm} 244$ and $\mathrm{Cf} 252$.

In this paper, we developed and applied a statistical approach to analyzing the relationship between proportion TRU and gross alpha activity. Obviously the analysis of other pairwise relationships (for instance, between Cf252 and gross alpha activity, proportion TRU and some beta/gamma emitters) may be accomplished within this framework. The main objective of those analyses would be to reveal statistically significant dependencies between TRU components and isotopes (or group of isotopes) whose concentrations can be relatively easily measured with NDA techniques. 


\section{Acknowledgment}

The findings reported in this paper are the results of a team effort. The authors are very grateful to members of the REDC and Waste Examination and Assay Facility, and in particular to J. Bigelow, D. Hensley, R. Schreiber, R. Taylor, and J. Trabalka, for their help, constructive criticism, and support of our work. We also thank S. Poteet, who took on the responsibility of converting our notes and thoughts into a readable text.

\section{References}

[1] Transuranic Waste Quality Assurance Program Plan, Rev. 0, CAO-944-1010, U.S. Department of Energy, Carlsbad, New Mexico, April 1995.

[2] Waste Acceptance Criteria for the Oak Ridge Reservation, ES/WM-10, Energy Systems Waste Management Organization, Martin Marietta, Oak Ridge, Tennessee, July 1994.

[3] TRU Waste Acceptance Criteria for the Waste Isolation Pilot Plant, WIPP-DOE069, Rev. 5, U.S. Department of Energy, Washington, D.C.

[4] Review of the Final Draft Transuranic Waste Characterization Acceptable Knowledge Guidance Document, Department of Energy Memorandum, U.S. Department of Energy, Carlsbad,-New Mexico, July 1995.

[5] "Waste Acceptance Criteria for the Waste Isolation Pilot Plant" DOE/WIPP-069, Revision 5, April 1996.

[6] Schultz, F. J., and Caldwell, J.T., 1991. DOE Assay Methods Used for Characterization of Contact-Handled Transuranic Waste, ORNL-6485, Oak Ridge, Tennessee.

[7] Hensley, D, 1995 (a) Source Imaging in the APNEA System, pp. 85-108, (b) Autonomous Matrix Identification by APNEA System, pp. 139-160, In "Proceedings 
of 4th Nondestructive Assay and Nondestructive Examination Waste Characterization Conference".

[8] Schultz, F. J., Vourvopoulos, G., Womble, P. C., Roberts, M. L., 1995, A Feasibility Study for a LINAC-Based Transuranic Waste Characterization System. Journal of Radioanalytical and Nuclear Chemistry, Articles, Vol. 193, No. 2, pp. 369-375.

[9] Reilly, D., Ensslin, N., and Smith, K., 1991, "Passive Nondestructive Assay of Nuclear Materials", Office of Nuclear Regulatory Research, U. S. Nuclear Regulatory Commission, Washington, D. C.

[10] Stewart, R.C., Dickerson, L.S., Joost, S.F., Osucha, D.C., 1989, Remote-Handled Transuranic Solid Waste Characterization Study: Oak Ridge National Laboratory, ORNL/TM-11050, Oak Ridge, Tennessee.

[11] Chattin, F.R., King, L.J., Peishel, F.L., 1976. Remote Processing Practices and Equipment in the Transuranium Processing Plant after 10 years of Operation, in "Proceedings of the 24-th Conference on Remote Systems Technology", pp. 118-129.

[12] Collins, E.D., and Bigelow, J.E., 1976. Chemical Process Engineering in the Transuranium Processing Plant, in "Proceedings of the 24-th Conference on Remote Systems Technology", pp. 130-139.

[13] Standard Test Method for Determination of Plutonium Isotopic Composition by Gamma - Ray Spectrometry, C 1030-89, ASTM, October 1989.

[14] Alpha Spectrometry with the ND9900 Data Acquisition System, AC-MM-7003115, Oak Ridge, September 1993.

[15] Moore, F. L. (1963), Alpha Spectrometry of Transuranium (TRU) Process Solutions, Silicon Diode Detector Method, Method NO. 9 062304, Oak Ridge.

[16] Kalbfliesch, J. D. and Prentice, R. L., 1980. The Statistical Analysis of Failure Time Data, New York, Wiley. 
[17] SAS/STAT, 1990. User's Guide, Version 6, Vols. 1 and 2, SAS Institute, Inc.

[18] Frome, E. L., Kutner, M. H., and Beauchamp, J. J., 1973, Regression Analysis of Poisson-Distributed Data, Journal of the American Statistical Association, 68, 935-940.

[19] Seber, G. A. F., and Wild, C. J., 1989, “Nonlinear Regression”, New York, Wiley.

[20] Johnson, N. I. and Kotz, S., 1970, "Continuous Univariate Distributions - 1." New York, Houghton Mifflin Company.

[21] Nguyen, L., 1996, Measurements of Fission and Activation Products for ORNL Transuranic Waste Characterization, TM-13292, ORNL, Oak Ridge, to be published. 


\section{Appendix 1. Data}

$-35-$ 
TABLE A1

THE 1st SET OF OBSERVATIONS (RH SERIES, 34 SAMPLES)

\begin{tabular}{|c|c|c|c|c|c|c|c|}
\hline Analysis & & RHQAA & RII9EE & RH9KK & RIIYLL & RHYOQ & nHess \\
\hline Ciruss Alpha & (Bq/(otal) & $911:+03+1 . \quad 1.9 E+02$ & $6.2 \mathrm{E}+05+1-7.4 \mathrm{E}+03$ & $3.8 E+08+1 . \quad 1.0 E+03$ & $1.2 E+04 \% 2.5 E+02$ & $1.9 E+0 S+1.2 .5 E+03$ & $61 E+04+1.1 .1 E+03$ \\
\hline \multicolumn{8}{|c|}{ Alntriemiliten } \\
\hline${ }^{214} \mathrm{Cm}$ & (Bu/total) & $831:+03+1 . \quad 8.3 E+02$ & $6.0 \mathrm{E} 105+1 . \quad 60 \mathrm{E}+04$ & $3.2 \mathrm{E}+04+1 . \quad 32 \mathrm{E}: 03$ & $1.1 E+144 \%$ I.1E103 & $1.8 E+05+1 . \quad 1.2 E+04$ & S. $12 E+04+1.5 .20 E+03$ \\
\hline $2+1 \mathrm{Pu}$ & (Bu/total) & $1.71: 102+1-3.4 E+01$ & $\cdot 0.2 \mathrm{E}+03$ & 8.5E+02 +f- 1.7E+02 & $<1.2 E \cdot n 2$ & $<1.9 \mathrm{E}+03$ & $1 \mathrm{EE+02}+1 \cdot \quad 2.2 E+0 \mid$ \\
\hline 211241 Pu & (By/lolal) & $<9$ 11:101 & $62 E+02+1-\quad 1.2 E+02$ & $5.8 E+01+1-\quad 1.2 E+01$ & $<1.2 \mathrm{E} 102$ & S.7E+02 +/- I.1E+02 & $1.2 E+02+1.2 .4 E+01$ \\
\hline${ }^{241} \mathrm{Am}$ & (Bq/lotal) & 1.71: $+02+1-5.4 E+00$ & $2.8 E 103+1-2.2 E+01$ & $2.5 E+02+1.6 .7 E+00$ & S.5E+02 $\% \quad 1.5 E+01$ & $5.6 E+03+1.2 .0 E+01$ & $6.2 E+02+1-1.1 E+01$ \\
\hline${ }^{241} \mathrm{Am}$ & (Bu/(otal) & $1.11:+01+122.0 E+00$ & $2.1 E+02+1.6 .9 E+00$ & $1.1 E+01+2.3 E+00$ & $4.2 E+01 \%$ 4.7E+00 & $1.2 \mathrm{E}+02+1-1.3 \mathrm{E}+00$ & $36 E+01+1.3 .2 E+00$ \\
\hline${ }^{252} \mathrm{Cf}$ & (Ily/iotal) & $211: 102+1.4 .65+01$ & $3.15:+03 * 6.2 E+02$ & $27 E+03+1.531: 102$ & 1.85:102 1\%. 3.6E:01 & $3.4 E+03+1-6.0 E+02$ & $12 E+03+1 . \quad 2.3 E+02$ \\
\hline${ }^{212} \mathrm{Cm}$ & (II)/total) & $141:+02+1-3.8 E+01$ & $16 E+04+1 \% 32 E+03$ & $2.2 E+03+1-4.4 E+102$ & $1.2 E 102+24 E+01$ & $4.0 E+03+1 . \quad 0 E+02$ & $7.3 E+02+1 \cdot \quad 1.5 E+02$ \\
\hline${ }^{24 .} \mathrm{cm}$ & (Bu/tuta) & $-911: \cdot 01$ & $62 E+02 \%$ । 2E+02 & $<5.8 E+02$ & < | 2E,n2 & $<19 E+03$ & $<6.1 E+02$ \\
\hline \multicolumn{8}{|c|}{ Beta/sammegarillers } \\
\hline "N" Co & (Bq/total) & $\leq 741:+100$ & 2.1E+01 & $<58 E+00$ & $<26$ : 104 & $<2.4 E+01$ & $<$ | SEtot \\
\hline IIn: Ru & (Ily/total) & $111: 102+1.2 .51:+111$ & I $11:+03+1.685 .101$ & 1.t1:+02 4/ 2 s1:101 & $4.71: 1113 \% \quad 1.06+02$ & $90 E+02+1 \% 9.0 E+01$ & S IE+02 +1\% 4.7E+01 \\
\hline 1114010 & (By/total) & - $\$ 71: 100$ & $1.21: 102+1 . \quad 49 E+00$ & $<62 E: 100$ & $1.21: 1102 \quad 1 \% \quad 53 \mathrm{E} \cdot 00$ & $11 \mathrm{E}: 02+1-5.2 E+00$ & $\therefore$ 10E+01 \\
\hline${ }^{126} \mathrm{sb}$ & (Hy/lotal) & $\therefore 2(11:+0)$ & $47 t+02+1 . \quad 1.7 E+01$ & | 2E,02 $1 / 6.3 E+00$ & S6E102 \% I \&E+01 & $3.5 \mathrm{E}+02+1 . \quad 1.6 \mathrm{E}+01$ & $3.2 E+02+1 . \quad 1.2 E+01$ \\
\hline "II cs & (Biplotal) & $\mid(1): 101+1 . \quad 1.6 E+1 \times 1$ & $19 E+02+1 \cdot 5.2 E+00$ & $|9 E+0|+\%$ | $8 E+00$ & $18 F: 102 \% 5.8 E+00$ & $1.4 E+02+1 . \quad 9.2 E+00$ & I IE+02 +1. 3.9E+00 \\
\hline "I's & $\left.(\mid)_{1} /(n+n)\right)$ & ) $81: 101+4.4 .91:+(K)$ & | 41:103 1\% | 7E:101 & | 81:102 11. \$ s1:100 & $1.61: 1(1) 3 \%$ 1\% $1.6 \mathrm{E} 101$ & $1.3 E+03+\%$ | SE+01 & $70 E+02+1 \% \quad 1.2 E+01$ \\
\hline "II le & (lisplutal) & $221: 102+1 \%$ 1.4E+01 & $261: 103+1 . \quad 4.3 E+01$ & | 3E:102 $1 \%$ | 21:101 & $1.81: 103 \quad 1 \% \quad 3.7 E+01$ & $20 \mathrm{O}: 03+1 . \quad 40 E+01$ & $1.7 E+03+1 . \quad 3.2 E+01$ \\
\hline 112 l:u & (Isy/total) & $\cdot 34 t: 1 n 1$ & 3I::102 +1. I.|E+0\} & $<278: 01$ & $1.6 \mathrm{E}+102 \% 7.6 \mathrm{E}+\infty 0$ & $1.5 E+02+4.5 E+01$ & $28 E+02+1 \cdot 72 E+00$ \\
\hline "I:u & (lly't(t)tal) & $151: 0(12+1 \%$ (.01:+1(x) & I נ1:103 +1 | I5:101 & 1 18:102 \% 3 11:100 & $8.31: 102+1.906: 100$ & $2.28+03+1 \%$ I.4E+01 & $795+02+1.8 .2 E+00$ \\
\hline Iss liu & (H)/lotal) & $911: 101+1.0 .18 E(0)$ & $6|E E+02+1 \cdot 6.1 E+0|$ & $5.1 E+01+1 \cdot 3.6 E+00$ & $4.3 \mathrm{t}+02 \quad 1 \% \quad 1.0 \mathrm{E}+01$ & $3.3 E+03+1-1.8 E+01$ & $3.3 E+02+1 . \quad 9.2 E+00$ \\
\hline${ }^{2 N} \mathrm{~Np}$ & (11)/lotal) & $111: 001+1.3 .5 E(0)$ & $5.3 E+02+1 \cdot 2.0 E+01$ & $<\mid$ SE+0| & $<4.4 E+01$ & $3.2 E+02+1 . \quad 5.0 E+01$ & $10 E+02+\%$ 2.4E+01 \\
\hline
\end{tabular}




\begin{tabular}{|c|c|c|c|c|c|c|c|}
\hline Analysis & & RHID & RH9E & RH9F & RHIJ & RII9M & RII9A \\
\hline Gross Alpha & (Bq/total) & $4.8 \mathrm{E}+04+1-\quad 1.2 \mathrm{E}+03$ & $2.6 \mathrm{E}+04+1-4.9 \mathrm{E}+02$ & $1.0 \mathrm{E}+05+1-\quad 1.9 \mathrm{E}+03$ & $5.2 E+04+1-1.1 E+03$ & $1.0 E+05+1-1.6 E+03$ & $1.1 \mathrm{r}+04+1-2.9 \mathrm{E}+02$ \\
\hline \multicolumn{8}{|c|}{ Alpha emilters } \\
\hline${ }^{2+4} \mathrm{Cm}$ & (Bq/total) & $4.6 \mathrm{E}+04+1.4 .6 \mathrm{E}+03$ & $2.4 \mathrm{E}+04+1.2 .4 \mathrm{E}+03$ & $9.7 \mathrm{E}+04+1.9 .7 \mathrm{E}+03$ & $4.2 \mathrm{~L}+04+1-4.2 \mathrm{E}+03$ & $9.3 E+04+/-9.3 E+03$ & $1.01:+04+1-1.01:+03$ \\
\hline${ }^{2.18} \mathrm{Pu}$ & (Bq/total) & $<4.8 \mathrm{E}+02$ & $3.0 \mathrm{E}+02+1-6.0 \mathrm{E}+01$ & $<1.0 \mathrm{E}+03$ & $2.7 E+02+1-5.4 E+01$ & $1.0 \mathrm{E}+02+1-2.0 \mathrm{E}+01$ & $1.3 E+02+/-2.6 E+01$ \\
\hline 2392+1" $\mathrm{Pu}$ & (Bq/total) & $<4.8 \mathrm{E}+02$ & $<2.6 \mathrm{E}+02$ & $5.0 \mathrm{E}+02+1-1.0 \mathrm{E}+02$ & $<5.2 \mathrm{E}+02$ & $4.0 \mathrm{E}+02+1.8 .0 \mathrm{E}+01$ & $<1.1 E+02$ \\
\hline${ }^{2 N 1} \mathrm{Am}$ & (Bq/total) & $1.0 \mathrm{E}+03+1-1.8 \mathrm{E}+01$ & $3.5 \mathrm{E}+02+1-9.0 \mathrm{E}+00$ & $4.6 \mathrm{E}+02+1-2.3 \mathrm{E}+01$ & $7.2 \mathrm{E}+02+/-1.9 \mathrm{E}+01$ & $1.4 \mathrm{E}+03+1-2.2 \mathrm{E}+01$ & $2.2 \mathrm{E}:+02+1-\quad 6.3 \mathrm{E}:+00$ \\
\hline${ }^{247} \wedge \mathrm{m}$ & (Bq/total) & $2.8 \mathrm{E}+01+1 \cdot \quad 6.0 \mathrm{E}+00$ & $3.2[E+01+1-5.0[5+00$ & $2.4 \mathrm{~F}+0 \mathrm{I}+1-6.4 \mathrm{E}+000$ & $2.91:+01+1-5.71:+00$ & $8.91:+01+/-9.01:+100$ & $5.11: 1001+1 /-1.81: 100)$ \\
\hline${ }^{232} \mathrm{Ct}$ & (Bq/tolal) & $8.8 \mathrm{E}+02+1 . \quad 1.9 \mathrm{E}+02$ & $7.2 E+02+1-\quad 1.41:+02$ & $8.11 \mathrm{E}+02+1-1.6 \mathrm{E}+02$ & $7.11: 103+1-1.41:+103$ & $3.21:+103+1-6.41: 102$ & $2.81:+(121 /-5.71: 101$ \\
\hline${ }^{2+2} \mathrm{Cm}$ & (Bq/total) & $8.0 \mathrm{E}+02+1-\quad 1.6 \mathrm{E}+02$ & $1.7 \mathrm{E}+02+1 \cdot \quad 3.3 \mathrm{E}+01$ & $5.9 \mathrm{E}+02+1-1.2 \mathrm{E}+02$ & $2.21:+03+/-4.41:+02$ & $2.0 \mathrm{E}+03+1-4.0 \mathrm{E}: 02$ & $1.71 \mathrm{k}+02+1-3.41 \mathrm{l}+01$ \\
\hline${ }^{2+16} \mathrm{Cm}$ & (Bq/total) & $<4.8 \mathrm{E}+02$ & $<2.6 \mathrm{E}+02$ & $4.0 \mathrm{E}+02+/-8.0 \mathrm{E}+01$ & $<5.2 \mathrm{r}:+02$ & $<1.0 \mathrm{E}+03$ & $<1.1 E+02$ \\
\hline \multicolumn{8}{|c|}{ Beta/gamma emillers } \\
\hline (1) Co & (Bq/total) & $3.0 \mathrm{E}+01+1-5.0 \mathrm{E}+00$ & $<1.4 \mathrm{E}+01$ & $<1.9 E+01$ & $<2.11: 101$ & $2.01 \sum+01+1-5.01 j+00$ & $5.01:+00+1-2.31:+00$ \\
\hline${ }^{\mathbf{H} \mathbf{K}_{\mathbf{B}}} \mathbf{R u}$ & (Bq/total) & $1.8 E+03+1-8.5 E+01$ & $2.3 \mathrm{E}+02+1-4.1 \mathrm{E}+01$ & $4.6 \mathrm{E}+02+1-8.6 \mathrm{E}+01$ & $1.91 \vdots+03+1-1.11 \vdots+102$ & $7.61:+02+1-9.0 F+01$ & $1.5 E+(02+/-2.8 E:+(1) 1$ \\
\hline${ }^{110 \mathrm{~m}} \mathrm{Ag}$ & (Bq/total) & $<1.4 \mathrm{E}+01$ & $3.9 E+01+1-3.7 E+00$ & $4.6 \mathrm{E}+02+1-9.0 \mathrm{E}+100$ & $<2.21:+(0)$ & $<2.31:+1) 1$ & $<7.01:+(00)$ \\
\hline${ }^{125} \mathrm{Sb}$ & (Bq/total) & $9.7 \mathrm{E}+01+1-1.8 \mathrm{E}+01$ & $9.9 \mathrm{E}+01+1-1.3 \mathrm{E}+01$ & $<5.61:+01$ & $2.91: 1(12+1-2.61:+01$ & $5.31 ;+02+/-2.61 ;+101$ & $<2.11:+01$ \\
\hline${ }^{114} \mathrm{Cs}$ & (Bq/total) & $8.4 \mathrm{E}+01+1-5.7 \mathrm{E}+00$ & $4.1 E+0 \mid+1-4.3 E+100$ & $9.9 E+01+1-6.6 E+00$ & $201:+12+1-8.11:+01)$ & $2.11:+102+1-8.51:+110$ & $1.71:+01+1-2.01:+00$ \\
\hline${ }^{137} \mathrm{Cs}$ & (Bq/total) & $5.7 \mathrm{E}+02+1 \cdot \quad 1.5 \mathrm{E}+01$ & $6.2 \mathrm{E}+02+1-\quad 1.4 \mathrm{E}+01$ & $1.2 \mathrm{E}+03+1-2.0 \mathrm{E}+01$ & $1.8 \mathrm{E}+03+1-2.4 \mathrm{E}+01$ & $25[:+03+1-2.9[:+01$ & $1.91:+02+1-6.01:+00$ \\
\hline${ }^{H+H} \mathrm{Ce}$ & (Bq/total) & $5.2 \mathrm{E}+02+/-3.2 \mathrm{E}+01$ & $3.5 E+02+1-2.7 E+01$ & $8.91:+02+/-5.0 \mathrm{E}+01$ & $1.21:+03+1-5.5 E:+01$ & $1.91:+03+1-5.4 \mathrm{E}+01$ & $2.1 \mathrm{E}+(02+/-\quad 1.51:+01$ \\
\hline${ }^{132}$ Eu & (Bq/total) & $<7.4 \mathrm{E}+01$ & $<6.5 \mathrm{E}+01$ & $<5.3 \mathrm{E}+01$ & $<8.81:+01$ & $4.61:+02+1-\quad 1.6 \mathrm{I}:+01$ & $<3.61:+01$ \\
\hline ISA Fit & (Bq/total) & $4.3[:+02+1-1 .(0]:+01$ & $2.8[E+02+1-8.11:+110$ & $2.4 l i+02+1-|| l i+() \mid$. & $4.71: 102+1 /-1.41: 101$ & $1.61:+(1) 3+1-\quad 1 .(1):+(0) 1$ & $1.41: 1021 /-3.61: 1010$ \\
\hline iss liu & (Bq/total) & $4.2 \mathrm{E}+02+1-\quad 1.1 \mathrm{E}+01$ & $1.3 \mathrm{E}+02+1-7.4 \mathrm{E}+00$ & $2.5 \mathrm{E}+02+1-1.4 \mathrm{E}+01$ & $3 .(11: 1112+1-1.61:+01$ & $7.81 \vdots+(1) 2+1-\quad 1.71 \vdots+01$ & $1.21:+02+1-4.11 i+00$ \\
\hline${ }^{2313} \mathrm{~Np}$ & (Bq/total) & $<5.2 \mathrm{E}+01$ & $<3.3 \mathrm{E}+01$ & $9.9 \mathrm{E}+01+1-2.0 \mathrm{E}+01$ & $<5.21:+01$ & $1.3[E+02+/-3.4 E+01$ & $<1.9 \mathrm{l} \mathrm{i}+(0)$ \\
\hline
\end{tabular}




\begin{tabular}{|c|c|c|c|c|c|c|c|}
\hline Analysis & & RH9C & RH9G & RH9H & RHI9I & RII9K & RIIYI. \\
\hline Gross Alpha & (Bq/total) & $3.5 \mathrm{E}+04+/-\quad 1.0 \mathrm{E}+03$ & $2.9 \mathrm{E}+04+1-5.2 \mathrm{E}+02$ & $5.1 E+04+/-1.2 E+03$ & $6.3 \mathrm{E}+04+1 \cdot \quad 1.4 \mathrm{E}+03$ & $1.1 \mathrm{E}+0 \mathrm{~S}+1-2.0 \mathrm{E}+03$ & $6.0 \mathrm{E}+04+1-\quad 1.4 \mathrm{~T}:+03$ \\
\hline \multicolumn{8}{|l|}{$\frac{\text { Alpha emilters }}{24 t}$} \\
\hline${ }^{24} \mathrm{Cm}$ & (Bq/total) & $3.3 E+04+1-3.3 E+03$ & $2.8 \mathrm{E}+04+1-2.8 \mathrm{E}+03$ & $4.9 \mathrm{E}+04+1-4.9 \mathrm{E}+03$ & $6.0 \mathrm{E}+04+1 \cdot 6.0 \mathrm{E}+03$ & $1.0 \mathrm{E}+05+1-\quad 1.0 \mathrm{E}+04$ & $5.5 E+04+/-5.5 E+03$ \\
\hline${ }^{27 k} \mathrm{Pu}$ & (Bq/total) & $4.2 \mathrm{E}+02+1-\quad 8.3 \mathrm{E}+01$ & $8.0 \mathrm{E}+02+1 . \quad 1.6 \mathrm{E}+02$ & $2.9 \mathrm{E}+01+1-5.8 \mathrm{E}+00$ & $3.5 \mathrm{E}+01+1.7 .0 \mathrm{E}+00$ & $6.8 \mathrm{E}+02+1-1.4 \mathrm{E}+02$ & $5.0 \mathrm{E}+02+/-1.0 \mathrm{E}+02$ \\
\hline $2132+11 \mathrm{Pu}$ & (Bq/total) & $<3.5 \mathrm{E}+02$ & $2.6 \mathrm{E}+02+1-5.2 \mathrm{E}+01$ & $<5.1 \mathrm{E}+02$ & $2.5 \mathrm{E}+02+1-5.0 \mathrm{E}+01$ & $4.4 E+02+/-8.8 E+01$ & $3.01 \mathrm{E}+02+1-6.0 \mathrm{E}+01$ \\
\hline${ }^{2.11} \mathrm{Am}$ & (Bq/total) & $7.4 \mathrm{E}+02+1-1.1 \mathrm{E}+01$ & $1.6 \mathrm{E}+02+1 \cdot 5.4 \mathrm{E}+00$ & $9.4 \mathrm{E}+02+1-1.2 \mathrm{E}+01$ & $9.1 E+02+1-1.3 E+01$ & $1.3 \mathrm{E}+03+1-1.6 \mathrm{E}+01$ & $8.2 \mathrm{E}+02+1-1.2 \mathrm{E}+01$ \\
\hline${ }^{2+1} \mathrm{Am}$ & (Bq/total) & $2.1 \mathrm{E}+01+1-2.6 \mathrm{E}+00$ & $8.9 \mathrm{E}+00+1.2 .4 \mathrm{E}+00$ & $4.6 \mathrm{E}+01+1-3.7 \mathrm{E}+00$ & $4.2 \mathrm{E}+01+1-3.7 \mathrm{E}+00$ & $8.1 E+01+/-6.2 E+00$ & $3.4 \mathrm{E}+01+1-4.4 \mathrm{E}+00$ \\
\hline${ }^{252} \mathrm{Cr}$ & (Bq/total) & $6.6 \mathrm{E}+02+1-1.3 \mathrm{E}+02$ & $1.5 \mathrm{E}+02+1-3.1 \mathrm{E}+01$ & $7.0 \mathrm{E}+02+1-1.4 \mathrm{E}+02$ & $9.4 \mathrm{E}+02+1-1.9 \mathrm{E}+02$ & $3.1 E+03+1-6.1 E+02$ & $1.9 \mathrm{E}+03+/ .3 .8 \mathrm{E}+02$ \\
\hline${ }^{242} \mathrm{Cm}$ & (Bq/total) & $5.3 \mathrm{E}+02+1-1.1 \mathrm{E}+02$ & $1.1 \mathrm{E}+02+1-2.2 \mathrm{E}+01$ & $4.3 \mathrm{E}+02+1-8.5 \mathrm{E}+01$ & $7.6 \mathrm{E}+02+/=1.5 \mathrm{E}+02$ & $2.4 E+03+1-4.9 E+02$ & $1.3 \mathrm{E}+03+1 \cdot 2.6 \mathrm{E}+02$ \\
\hline${ }^{2461} \mathrm{Cm}$ & (Bq/total) & $<3.5 \mathrm{E}+02$ & $<2.9 \mathrm{E}+02$ & $<5.1 \mathrm{E}+02$ & $<6.3 \mathrm{E}+02$ & $3.3 \mathrm{E}+02+1-6.6 \mathrm{E}+01$ & $<6.0 \mathrm{E}+02$ \\
\hline \multicolumn{8}{|c|}{ Beta/gamma emitters } \\
\hline${ }^{(x)} \mathrm{Co}$ & (Bq/total) & $<8.0 \mathrm{E}+00$ & $8.7 \mathrm{E}+00+1-1.9 \mathrm{E}+00$ & $1.2 \mathrm{E}+01+1-2.6 \mathrm{E}+00$ & $2.2 E+01+/-3.7 \mathrm{E}:+00$ & $3.51:+101+1.4 .1 E+100$ & $<1.11:+01$ \\
\hline${ }^{\prime \prime K_{i}} \mathrm{Ru}$ & (Bq/total) & $3.1 \mathrm{E}+02+/-3.9 \mathrm{E}+01$ & $1.2 \mathrm{E}+02+1.2 .7 \mathrm{E}+01$ & $4.5 \mathrm{E}+02+1-\quad 4.7 \mathrm{E}+01$ & $4.9 E+(12+1-5.1 E+01$ & $4.2 \mathrm{E}+02+1 . \quad 6.0 \mathrm{E}+01$ & $3.2 E+02+1.5 .31:+01$ \\
\hline 116!1" $\mathrm{Ag}$ & (Bq/total) & $<1.1 \mathrm{E}+01$ & $2.3 \mathrm{E}+01+1-2.1 \mathrm{E}+00$ & $6.2 \mathrm{E}+01+1-3.2 \mathrm{E}+00$ & $4.7 \mathrm{E}+01+/ 2 \quad 3.3 \mathrm{E}+00$ & $<1.4 E+01$ & $<1.28+01$ \\
\hline${ }^{123} \mathrm{Sb}$ & (Bq/total) & $1.4 \mathrm{E}+02+1-9.0 \mathrm{E}+00$ & $5.0 \mathrm{E}+01+1-7.6 \mathrm{E}+00$ & $1.5 \mathrm{E}+02+/-\quad 1.0 \mathrm{E}+01$ & $3.2 \mathrm{E}+02+1-1.2 \mathrm{I}:+01$ & $3.0 \mathrm{E}+02+1-1.5[i+01$ & $<4.01 i+01$ \\
\hline${ }^{134} \mathrm{Cs}$ & (Bq/total) & $3.3 \mathrm{E}+01+1-3.1 \mathrm{E}+00$ & $1.9 \mathrm{E}+01+1-2.0 \mathrm{E}+00$ & $4.9 \mathrm{E}+01+1-3.0 \mathrm{E}+00$ & $8.9 E+01+1-3.8 E+100$ & $1.3 \mathrm{E}+02+1-5.0 \mathrm{E}+00$ & $5.8 k+01+/-4.31:+00$ \\
\hline${ }^{137} \mathrm{Cs}$ & (Bq/total) & $3.7 \mathrm{E}+02+1-8.0 \mathrm{E}+00$ & $1.7 \mathrm{E}+02+1-6.0 \mathrm{E}+00$ & $5.0 \mathrm{E}+02+1-9.3 \mathrm{E}+00$ & $9.9 \mathrm{E}+02+1-1.2 \mathrm{E}+01$ & $i .4 \mathrm{E}+03+1-1.7 \mathrm{E}+01$ & $6.5 E+02+/-1.1 \mathrm{E}+01$ \\
\hline${ }^{\mathrm{H} H} \mathrm{Ce}$ & (Bq/total) & $5.2 \mathrm{E}+02+1-2.1 \mathrm{E}+01$ & $1.0 \mathrm{E}+02+\%-1.2 \mathrm{E}+01$ & $4.0 \mathrm{E}+02+1-2.1 \mathrm{E}+01$ & $5.5 \mathrm{E}+02+1-2.3 \mathrm{E}+01$ & $2.6 \mathrm{E}+03+1 \cdot \quad 4.0 \mathrm{E}+01$ & $1.31:+03+/-2.7 E+01$ \\
\hline${ }^{152}$ Eu & (Bq/total) & $8.5 \mathrm{E}+01+1-5.7 \mathrm{E}+00$ & $<3.4 \mathrm{E}+01$ & $<4.4 \mathrm{E}+01$ & $8.5 \mathrm{E}+01+/-1.4 \mathrm{E}+01$ & $5.9 \Gamma+02+1-9.05+00$ & $2.8 \mathrm{E}+02+1-\quad 1.51 \mathrm{i}+01$ \\
\hline${ }^{154}$ E. & (Bq/total) & $4.0 \mathrm{E}+02+/-5.7 \mathrm{E}+00$ & $1.8 \mathrm{E}+02+1-4.5 \mathrm{E}+00$ & $6.5 \mathrm{E}+02+1 \cdot 6.81 \mathrm{I}+100$ & $5.91:+(12+1-8 .(1):+100)$ & $1.81:+03+1-1.25:+01$ & $8.8[:+112+1-8.61: 100$ \\
\hline${ }^{195}$ Eu & (Bq/total) & $3.7 \mathrm{E}+02+1-5.9 \mathrm{E}+00$ & $1.1 \mathrm{E}+02+/-4.2 \mathrm{E}+00$ & $4.5 \mathrm{E}+02+1 \cdot \quad 8.0 \mathrm{E}+00$ & $3.7 \mathrm{E}+02+1-7.4 \mathrm{E}+00$ & $8.4 \mathrm{E}+02+1-1.2 \mathrm{E}+01$ & $4.11:+02+1-\quad 1.2 E+01$ \\
\hline${ }^{219} \mathrm{~Np}$ & (Bq/total) & $5.9 \mathrm{E}+01+/-1.2 \mathrm{E}+01$ & $<1.9 \mathrm{E}+01$ & $<9.0 \mathrm{E}+01$ & $7.4 \mathrm{E}+01+1-1.6 \mathrm{E}+(01$ & $1.3 \mathrm{E}+02+1-1.9 \mathrm{E}+01$ & $7.2 \mathrm{E}: 01+1-\quad 1.8 \mathrm{r}:+01$ \\
\hline
\end{tabular}




\begin{tabular}{|c|c|c|c|c|c|c|c|}
\hline Analysis & & RHIN & RH9P & RH9Q & RII9S & RHIT & RHISU \\
\hline Gross Alpha & (Bq/1otal) & $1.9 \mathrm{E}+05+1-2.3 \mathrm{E}+03$ & $9.9 \mathrm{E}+04+\% \quad 1.9 \mathrm{E}+03$ & $8.7 \mathrm{E}+04+1-1.7 \mathrm{E}+03$ & $1.5 \mathrm{E}+05+\% .2 .4 \mathrm{E}+03$ & $1.6 \mathrm{E}+04+1-3.5 \varepsilon+02$ & $1.6 \mathrm{E}+04+1-3.5 E+02$ \\
\hline \multicolumn{8}{|c|}{ Alpha emitters } \\
\hline${ }^{2 H} \mathrm{Cm}$ & (Bq/lotal) & $1.7 \mathrm{E}+05+1 . \quad 1.7 \mathrm{E}+04$ & $9.4 \mathrm{E}+04+1-9.4 \mathrm{E}+03$ & $8.2 E+04+1-8.2 E+03$ & $1.5 E+05+1-1.5 E+04$ & $1.5 \mathrm{E}+04+1-\quad 1.5 \mathrm{E}+03$ & $1.5 E+04+1-1.5 E+03$ \\
\hline${ }^{23 \mathrm{M}} \mathrm{Pu}$ & (Bq/total) & $<1.9 \mathrm{E}+03$ & $<9.9 \mathrm{E}+02$ & $<8.7 \mathrm{E}+02$ & $<1.5 E+03$ & $3.9 E+02+1 \cdot 7.9 E+01$ & $<1.6 \mathrm{E}+02$ \\
\hline${ }^{2 . v n 2+11} \mathrm{Pu}$ & (Bq/lotal) & $1.1 \mathrm{E}+03+1.2 .3 \mathrm{E}+02$ & $4.0 \mathrm{E}+02+1-7.9 \mathrm{E}+01$ & $<8.7 \mathrm{E}+02$ & $1.5 E+02+1-3.0 E+01$ & $2.9 \mathrm{E}+02+1-5.8 \mathrm{E}+01$ & $<1.6 \mathrm{E}+02$ \\
\hline${ }^{211} \mathrm{Am}$ & (Bq/total) & $1.1 \mathrm{E}+04+1-5.4 \mathrm{E}+01$ & $2.2 \mathrm{E}+03+1.2 .6 \mathrm{E}+01$ & $3.0 \mathrm{E}+03+1-2.8 \mathrm{E}+01$ & $2.1 E+03+1-1.9 E+01$ & $8.7 E+01+1-1.9 E+01$ & $3.2 E+02+/-6.7 E+00$ \\
\hline${ }^{243} \mathrm{Am}$ & (Bq/lotal) & $1.0 \mathrm{E}+02+1-1.5 \mathrm{E}+01$ & $9.6 \mathrm{E}+01+1-7.1 \mathrm{E}+00$ & $4.3 E+01+1-7.1 E+00$ & $3.2 \mathrm{E}+02+/-8.6 \mathrm{E}+00$ & $7.5 \mathrm{E}+00+1-8.6 \mathrm{E}+00$ & $8.0 \mathrm{E}+00+1.2 .3 \mathrm{E}+00$ \\
\hline${ }^{232} \mathrm{Cr}$ & (Bq/total) & $2.9 \mathrm{E}+03+1-5.9 \mathrm{E}+02$ & $1.2 \mathrm{E}+03+1.2 .4 \mathrm{E}+02$ & $8.4 \mathrm{E}+02+1-1.7 \mathrm{E}+02$ & $3.9 \mathrm{E}+02+1-7.8 \mathrm{E}+01$ & $2.0 \mathrm{E}+02+1-4.0 \mathrm{E}+01$ & $3.2 \mathrm{E}+02+1-6.4 \mathrm{E}+01$ \\
\hline${ }^{212} \mathrm{Cm}$ & (Bq/total) & $2.4 E+03+1-4.7 E+02$ & $8.0 E+02+1-1.6 E+02$ & $1.6 \mathrm{E}+03+1-3.2 \mathrm{E}+02$ & $2.1 E+02+1.4 .2 E+01$ & $1.5 \mathrm{E}+02+1-3.0 \mathrm{E}+01$ & $2.9 \mathrm{E}+02+/-5.8 \mathrm{E}+01$ \\
\hline${ }^{2+16} \mathrm{Cm}$ & (Bq/lotal) & $<1.9 \mathrm{E}+03$ & $<9.9 \mathrm{E}+02$ & $<8.7 \mathrm{E}+02$ & $<1.5 \mathrm{E}+03$ & $1.6 \mathrm{E}+02+/-3.2 \mathrm{E}+01$ & $<1.6 \mathrm{E}+02$ \\
\hline \multicolumn{8}{|c|}{ Beta/gamma eminters } \\
\hline${ }^{(N)} \mathrm{Co}$ & (Bq/total) & $<2.2 \mathrm{E}+01$ & $2.7 E+01+1-5.5 E+00$ & $<1.4 \mathrm{E}+01$ & $3.7 E+01+1-3.81:+100$ & $<1.4 \mathrm{E}+01$ & $<1.0 \mathrm{E}+01$ \\
\hline${ }^{I} K_{K} R u$ & (Bq/total) & $9.1 \mathrm{E}+02+1-\quad 1.1 \mathrm{E}+02$ & $3.7 E+02+1-9.7 E+01$ & $<1.5 \mathrm{E}+02$ & $3.1 E+02+1-5.81:+01$ & $<1.0 \mathrm{E}+02$ & $1.9 \mathrm{E}+02+1-2.8 \mathrm{E}+01$ \\
\hline${ }^{1111 \mathrm{~mm}} \mathrm{Ag}$ & (Bq/lotal) & $<2.7 \mathrm{E}+01$ & $<1.9 E+01$ & $<1.7 \mathrm{E}+01$ & $<1.5 E+01$ & $5.0 \mathrm{E}+01+1-\quad 3.5 \mathrm{E}+00$ & $<7.0 \mathrm{E}:+100$ \\
\hline${ }^{125} \mathrm{Sb}$ & (Bq/total) & $8.8 E+02+/-3.1 E+01$ & $6.0 \mathrm{E}+02+1.2 .3 \mathrm{E}+01$ & $<5.01:+01$ & $1.91:+03+/-3.21 \vdots+01$ & $<3.01:+01$ & $<2.41:+01$ \\
\hline${ }^{M N} \mathrm{Cs}$ & (Bq/lotal) & $1.7 E+02+1-1.0 E+01$ & $1.0 \mathrm{E}+02+1.6 .5 \mathrm{E}+00$ & $2.8 \mathrm{E}+01+1-4.6 \mathrm{E}+00$ & $4.4 \mathrm{E}+(12+/-6.9 \mathrm{E}+00$ & $<\lceil .0 \mathrm{E}+0\rceil$ & $1.3 E+01+1-2.0 E++00$ \\
\hline${ }^{117} \mathrm{Cs}$ & (Bq/lotal) & $1.5 \mathrm{E}+03+1-2.4 \mathrm{E}+01$ & $1.2 E+03+1-2.0 E+01$ & $3.1 E+02+1-1.1 E+01$ & $6.9 E+03+/-3.3 E+01$ & $6.3 E+01+/ 25.5 E+00$ & $1.4 \mathrm{E}+02+1-5.1 \mathrm{E}+00$ \\
\hline${ }^{\text {WH } \mathrm{Ce}}$ & (Bq/lotal) & $1.9 \mathrm{E}+03+1-6.0 \mathrm{E}+01$ & $7.6 \mathrm{E}+02+1-4.1 \mathrm{E}+01$ & $5.9 \mathrm{E}+02+1-3.2 \mathrm{E}+01$ & $9.9 \mathrm{E}+01+1-2.4 \mathrm{E}+01$ & $1.2 \mathrm{E}+02+1-1.9 \mathrm{E}+01$ & $1.4 E+02+/ . \quad 1.4 k+01$ \\
\hline${ }^{132}$ Eu & (Bq/total) & $4.4 \mathrm{E}+02+1-1.6 \mathrm{E}+01$ & $<9.9 \mathrm{E}+01$ & $<1.6 \mathrm{E}+02$ & $1.1 \mathrm{E}+02+1-2.2 \mathrm{E}+01$ & $<4.8 \mathrm{E}+01$ & $<3.6 \mathrm{E}+01$ \\
\hline${ }^{154}$ Eu & (Bq/lotal) & $5.4 \mathrm{E}+03+1-2.7 \mathrm{E}+01$ & $9.7 \mathrm{E}+02+1-\quad 1.4 \mathrm{E}:+01$ & $1.4 E+03+1-1.5 E+01$ & $3.41:+(13+1-1.71:+01$ & $6.8 \mathrm{E}+01+1-4.1 \mathrm{t}:+100$ & $1.2 \mathrm{E}+(12+1=4.01:+(11)$ \\
\hline${ }^{135}$ Eu & (Bq/total) & $9.6 \mathrm{E}+03+1-4.0 \mathrm{E}+01$ & $3.9 \mathrm{E}+02+1-2.0 \mathrm{E}+01$ & $2.2 \mathrm{E}+03+1-\quad 1.8 \mathrm{E}+01$ & $1.31:+03+1-2.01:+02$ & $3.71:+01+1-4.9 E+00$ & $1.0 \mathrm{E}+02+1-3.9 \mathrm{E}+00$ \\
\hline${ }^{210} \mathrm{~Np}$ & (Bq//otal) & $5.2 \mathrm{E}+02+1-5.2 \mathrm{E}+01$ & $1.3 \mathrm{E}+02+1-1.5 \mathrm{E}+01$ & $1.6 \mathrm{E}+02+/-4.0 \mathrm{E}+01$ & $3.5 \mathrm{E}+02+1-5.3 \mathrm{E}+01$ & $<2.0 E+01$ & $<1.8 \mathrm{E}+01$ \\
\hline
\end{tabular}




\begin{tabular}{|c|c|c|c|c|c|c|c|}
\hline Analysis & & RHI9V & RH9O & RHI9R & RIIIB & RII213 & RII3I3 \\
\hline Gross Alpha & (Bq/total) & $5.9 \mathrm{E}+04+1-5.9 \mathrm{E}+02$ & $5.3 \mathrm{E}+03+/-\quad 1.3 \mathrm{E}+02$ & $2.2 \mathrm{E}+04+1-4.8 \mathrm{E}+02$ & $5.4 \mathrm{E}+04+1-1.2 \mathrm{E}+03$ & $6.3 \mathrm{E}+04+/-\quad 1.4 \mathrm{E}+03$ & $4.3 \mathrm{E}+05+/-5.6 \mathrm{E}+03$ \\
\hline \multicolumn{8}{|c|}{ Alpha emilters } \\
\hline${ }^{241} \mathrm{Cm}$ & (Bq/total) & $5.4 \mathrm{E}+04+1-5.4 \mathrm{E}+03$ & $3.4 \mathrm{E}+03+/-3.4 \mathrm{E}+02$ & $2.1 \mathrm{E}+04+\% \quad 2.1 \mathrm{E}+03$ & $5.4 \mathrm{E}+04+1-5.4 \mathrm{E}+03$ & $6.2 \mathrm{E}+04+1-\quad 6.2 \mathrm{E}+03$ & $4.3 €+05+/ . \quad 4.3 \mathrm{E}+04$ \\
\hline${ }^{211} \mathrm{Pu}$ & (Bq/total) & $3.6 \mathrm{E}+02+1-7.2 \mathrm{E}+01$ & $4.6 \mathrm{E}+02+1-9.1 \mathrm{E}+01$ & $2.4 \mathrm{E}+02+1-4.8 \mathrm{E}+01$ & $<5.4 \mathrm{E}+02$ & $<6.3 \mathrm{E}+02$ & $<4.35+03$ \\
\hline${ }^{219 / 2+11} \mathrm{Pu}$ & (Bq/total) & $3.5 \mathrm{E}+02+1-7.1 \mathrm{E}+01$ & $<5.3 \mathrm{E}+01$ & $<2.2 \mathrm{E}+02$ & $<5.4 \mathrm{E}+02$ & $<6.3 \mathrm{E}+02$ & $<4.3 \mathrm{~F}+03$ \\
\hline${ }^{2+1} \mathrm{Am}$ & (Bq/total) & $7.6 \mathrm{E}+02+/-\quad 1.7 \mathrm{E}+01$ & $5.4 \mathrm{E}+01+1-5.7 \mathrm{E}+00$ & $2.2 \mathrm{E}+02+1-6.3 \mathrm{E}+00$ & $3.8 \mathrm{E}+02+/-7.3 \mathrm{E}+00$ & $6.4 \mathrm{E}+02+1-9.3 \mathrm{E}+00$ & $3.9 E+03+/-2.1 E+01$ \\
\hline 24! $\mathrm{Am}$ & (Bq/total) & $6.9 \mathrm{E}+01+1 . \quad 7.2 \mathrm{E}+00$ & $4.0 \mathrm{E}+00+1-2.8 \mathrm{E}+00$ & $1.0 \mathrm{E}+01+/ 2.4 \mathrm{E}+00$ & $7.3 \mathrm{E}+00+1-2.3 \mathrm{E}+00$ & $2.6 \mathrm{E}+01+1-2.7 \mathrm{E}+00$ & $1.0 \mathrm{E}+02+1-3.4 \mathrm{E}+100$ \\
\hline${ }^{232} \mathrm{Cr}$ & (Bq/lotal) & $2.1 \mathrm{E}+03+\%-4.2 \mathrm{E}+02$ & $1.4 \mathrm{E}+03+\% \quad 2.8 \mathrm{E}+02$ & $3.9 \mathrm{E}+02+1-7.8 \mathrm{E}+01$ & $5.2 E+01+1-1.0 E+01$ & $7.5 \mathrm{E}+01+1-.1 .5 \mathrm{E}+01$ & $<4.31:+03$ \\
\hline${ }^{212} \mathrm{Cm}$ & (Bq/total) & $9.5 \mathrm{E}+02+1-1.9 \mathrm{E}+02$ & $<5.3 \mathrm{E}+01$ & $3.4 \mathrm{E}+02+1 . \quad 6.7 \mathrm{E}+01$ & $5.61:+01+1-1.115+01$ & $1.1 \mathrm{E}+02+1-2.3 \mathrm{E}+01$ & $<4.31:+03$ \\
\hline${ }^{245} \mathrm{Cm}$ & (Bq/totai) & $1.8 \mathrm{E}+02+1-3.5 \mathrm{E}+01$ & $<5.3 \mathrm{E}+01$ & $<2.2 \mathrm{E}+02$ & $<5.4 t:+02$ & $<6.35+02$ & $<4.3 \mathrm{E}+03$ \\
\hline \multicolumn{8}{|c|}{ Beta/gamma emilters } \\
\hline "so & (Bq/total) & $1.1 E+0 \mid+1-5.1 E+00$ & $<1.0 \mathrm{E}+01$ & $<1.3 \mathrm{E}+01$ & $<8 .(01:+1) 0$ & $<1.0 \mathrm{E}+01$ & $<7.0 \mathrm{E}+00$ \\
\hline${ }^{I ! K} \mathrm{Ru}$ & (Bq/total) & $5.7 \mathrm{E}+02+/-8.2 \mathrm{E}+01$ & $1.3 \mathrm{E}+03+1-\quad 7.1 \mathrm{E}+01$ & $1.6 \mathrm{E}+02+1-3.7 \mathrm{E}+01$ & $<4.5 \mathrm{E}:+01$ & $<5.6 \mathrm{E}+01$ & $<5.0 \mathrm{E}+01$ \\
\hline$" 111 \mathrm{~m} \mathrm{Ag}$ & (Bq/total) & $2.6 \mathrm{E}+01+\%-4.7 \mathrm{E}+00$ & $1.1 \mathrm{E}+03+1.9 .3 \mathrm{E}+00$ & $<8.4 \mathrm{E}+00$ & $<6.01:+00$ & $<6.08+00$ & $<5.01:+00$ \\
\hline${ }^{125} \mathrm{Sb}$ & (Bq/total) & $2.2 \mathrm{E}+02+1-\quad 1.8 \mathrm{E}+01$ & $1.9 \mathrm{E}+02+1-\quad 1.3 \mathrm{E}+01$ & $9.4 \mathrm{E}+01+/-8.2 \mathrm{E}+00$ & $<1.5 E+01$ & $<1.6 \mathrm{E}+01$ & $<1.3 \mathrm{E}+01$ \\
\hline${ }^{134} \mathrm{Cs}$ & (Bq/total) & $1.3 \mathrm{E}+02+1-6.6 \mathrm{E}+00$ & $<1.2 \mathrm{E}+01$ & $4.0 \mathrm{E}+01+1-2.7 \mathrm{E}+00$ & $<5.0 \mathrm{E}+00$ & $<6.0 \mathrm{~F}+00$ & $<5.0 \mathrm{E}+00$ \\
\hline${ }^{137} \mathrm{Cs}$ & (Bq/total) & $8.5 \mathrm{E}+02+1 \cdot \quad 1.7 \mathrm{E}+01$ & $9.9 \mathrm{E}+01+1-6.9 \mathrm{E}+00$ & $4.0 \mathrm{E}+02+1-8.1 \mathrm{E}+00$ & $3.1 E+01+1-3.51 E+00$ & $4.9 \mathrm{E}+01+/-3.6 \mathrm{E}+00$ & $2.1 \mathrm{E}+01+1-\quad 3.8 \mathrm{E}+00$ \\
\hline${ }^{\mathrm{HH}} \mathrm{Ce}$ & (Bq/total) & $1.3 \mathrm{E}+03+/-4.6 \mathrm{E}+01$ & $1.5 \mathrm{E}+02+1 \cdot \quad 1.9 \mathrm{E}+01$ & $2.5 \mathrm{E}+02+1-1.5 \mathrm{E}+01$ & $3.8 E^{2}+01+1-7.5 E+00$ & $6.4 \mathrm{E}+01+\% \quad 1.2 \mathrm{E}+01$ & $4.7 E+01+/-1.1 E+01$ \\
\hline${ }^{152}$ Eu & (Bq/total) & $1.4 \mathrm{E}+02+1-9.0 \mathrm{E}+00$ & $<3.4 \mathrm{E}+01$ & $5.3 \mathrm{E}+01+1-3.7 \mathrm{E}+00$ & $<2.7 \mathrm{E}+01$ & $<2.7 \mathrm{E}+0 \mathrm{l}$ & $<2.8 \mathrm{r}+01$ \\
\hline 154 Eu & (Bq/total) & 7.3E $+02+1-1.1 E+01$ & $<3.7 E+01$ & $1.9 \mathrm{~F}+02+1-4.31:+00$ & $1.01:+(12+1-3.41:+100$ & $9.71:+01+/-3.71:+1010$ & $4.81:+01+1-2.91:+(0)$ \\
\hline iss liu & (By/total) & $3.6 \mathrm{E}+02+1-1.2 \mathrm{E}+01$ & $3.35:+01+\%$ s. $1 \mathrm{~L}+100$ & $1.11 j+02+1.4 .71 i+00$ & $1.31:+102+1-4.51 ;+100$ & $1.41 j+02+1.4 .61 i+00$ & $6.61:+101+1-7.015+(0)$ \\
\hline${ }^{2.311} \mathrm{~Np}$ & (Bq/total) & $1.2 \mathrm{E}+02+1 \cdot \quad 2.6 \mathrm{E}+01$ & $<1.8 \mathrm{E}+01$ & $<2.0 \mathrm{E}+01$ & $4.3 \mathrm{E}+01+1-6.6 \mathrm{E}+00$ & $5.4 \mathrm{E}+01+/-5.9 \mathrm{E}+00$ & $2.3 E+02+/-8.7 E+00$ \\
\hline
\end{tabular}




\begin{tabular}{|c|c|c|c|c|c|c|c|c|c|}
\hline \multirow{2}{*}{$\begin{array}{l}\text { Analysis } \\
\text { Gross Alpha }\end{array}$} & \multirow[b]{2}{*}{ (Bq/total) } & \multicolumn{2}{|c|}{ RHI4D } & \multicolumn{2}{|c|}{ RH6C } & \multicolumn{2}{|c|}{ RHI7D } & \multicolumn{2}{|c|}{ RII8A } \\
\hline & & $8.1 \mathrm{E}+07+1-$ & $1.1 \mathrm{E}+06$ & $3.0 \mathrm{E}+06+1-$ & $3.0 \mathrm{E}+04$ & $2.7 \mathrm{E}+06+1-$ & $3.5 E+04$ & $2.4 E+08+1$ & $3.1 \mathrm{E}+06$ \\
\hline \multicolumn{10}{|l|}{ Alpha emilters } \\
\hline${ }^{244} \mathrm{Cm}$ & (Bq/total) & $6.9 E+07+1-$ & $6.9 E+06$ & $2.9 E+06+1-$ & $2.9 E+05$ & $2.5 E+06+1-$ & $2.5 E+05$ & $2.4 E+08+1$. & $2.4 E+07$ \\
\hline${ }^{211} \mathrm{Pu}$ & (Bq/total) & $<8.1 \mathrm{E}+05$ & & $<3.0 \mathrm{E}+04$ & & $<2.7 E+04$ & & $<2.4 \mathrm{E}+06$ & \\
\hline 2.39/2+11 $\mathrm{Pu}$ & (Bq/total) & $8.1 \mathrm{E}+04+1$ & $1.6 \mathrm{E}+04$ & $<3.0 \mathrm{E}+04$ & & $5.4 \mathrm{E}+03+1$ & $1.1 E+03$ & $<2.4 \mathrm{E}+06$ & \\
\hline${ }^{2+1} \mathrm{Am}$ & (Bq/total) & $1.1 E+07+1-$ & $1.0 \mathrm{E}+04$ & $8.8 \mathrm{E}+04+1$. & $1.1 \mathrm{E}+02$ & $2.3 E+04+1$ & $1.1 \mathrm{E}+02$ & $2.1 \mathrm{E}+05+1-$ & $5.2 \mathrm{E}+02$ \\
\hline${ }^{24 ! 3} \mathrm{Am}$ & (Bq/total) & $6.8 \mathrm{E}+04+1$. & $2.6 \mathrm{E}+03$ & $1.7 E+03+1-$ & $1.7 \mathrm{E}+01$ & $1.4 \mathrm{E}+03+1-$ & $2.8 E+01$ & $2.9 F+04+1$ & $2.05+02$ \\
\hline${ }^{232} \mathrm{Cr}$ & (Bq/total) & $1.6 \mathrm{E}+05+1-$ & $3.3 E+04$ & $1.6 \mathrm{E}+04+1-$ & $3.3 \mathrm{E}+03$ & $6.2 \mathrm{E}+04+1$. & $1.2 E+04$ & $6.4 \mathrm{E}+05+1-$ & $1.3 E+05$ \\
\hline${ }^{242} \mathrm{Cm}$ & (Bq/total) & $1.3 \mathrm{E}+06+1$. & $2.6 \mathrm{E}+05$ & $2.0 \mathrm{E}+04+1-$ & $3.9 E+03$ & $8.9 \mathrm{E}+04+/-$ & $1.8 \mathrm{E}+04$ & $3.21:+05+1$. & $6.5 F:+04$ \\
\hline${ }^{246} \mathrm{Cm}$ & (Bq/total) & $<8.1 E+05$ & & $<3.0 \mathrm{E}+04$ & & $1.1 \mathrm{E}+04+1$. & $2.2 \Gamma:+03$ & $<241:+106$ & \\
\hline \multicolumn{10}{|c|}{ Bela/gamma emillers } \\
\hline (A) $\mathrm{Co}$ & (Bq/total) & < 1.51: $: 03$ & & $2.7 E+02+1$ & $1.31:+01$ & $1.41:+103+1$. & $3.01:+01$ & $1.41:+113+1-$ & $951: 1(1) 1$ \\
\hline${ }^{\prime \prime *} R u$ & (Bq/total) & $<2.9 E+04$ & & $1.8 \mathrm{E}+04+1$. & $28 E+02$ & $3.7 \mathrm{E}+04+1-$ & $5.41:+02$ & $3.4 \mathrm{I}:+05+1-$ & $231: 1(13$ \\
\hline${ }^{18 m+1} \mathrm{Ag}$ & (Bq/total) & $1.21:+04+1$. & $9.7 E+02$ & $29 \mathrm{E}+03+1$. & $21 \mathrm{tE}+01$ & $975+112+\%$ & $3.11:+01$ & $116:+03+1-$ & $1.31: 1112$ \\
\hline${ }^{125} \mathrm{Sb}$ & (Bq/lotal) & $<8.1 \mathrm{E}+03+\%$ & & $4.5 E+04+\%$ & $2.3 E+03$ & $5.0 \mathrm{E}+03+1$. & $501: \div 02$ & I sl:t04 +/- & $691:+1) 2$ \\
\hline${ }^{\prime \prime} \mathrm{Cs}$ & (Bq/total) & $<2.9 \mathrm{E}+03+1$ & & $2.1 \mathrm{E}+03+/$ & $9.7 E+01$ & $1.18+04+1-$ & $5.31 \mathrm{i}+01$ & $841:+03+1$ & $1,61:+1) 2$ \\
\hline${ }^{137} \mathrm{Cs}$ & (Bq/total) & $3.1 E+04+\%$ & $1.5 \mathrm{E}+03$ & $2.0 \mathrm{E}+04+1$ & $5.8 \mathrm{E}+01$ & $8.3 E+04+1$. & $1.81:+02$ & $5.315+04+1-$ & $2.91:+12$ \\
\hline${ }^{1+4} \mathrm{Ce}$ & (Bq/total) & $4.1 \mathrm{E}+05+1-$ & $7,2 \mathrm{E}+03$ & $1.1 \mathrm{E}+04+1-$ & $1.3 \mathrm{E}+02$ & $4.2 \mathrm{E}+04+1-$ & $3.0 \mathrm{E}+112$ & $8.31:+04+1-$ & $1.3 \mathrm{l}:+03$ \\
\hline${ }^{132} \mathrm{Eu}$ & (Bq/total) & $4.1 \mathrm{E}+04+1-$ & $1.4 \mathrm{E}+03$ & $4.3 E+03+1-$ & $1.2 \mathrm{E}+02$ & $1.2 E+03+/-$ & $3.0 \mathrm{E}+02$ & $8.5 \mathrm{E}+03+1$. & $2.4 \mathrm{E}+02$ \\
\hline "SA Eu & (Bq/total) & $3.9 \mathrm{E}+06+1-$ & $1.3 \mathrm{E}+04$ & $1.8 \mathrm{E}+04+/-$ & $4.1 \mathrm{E}+01$ & $1.5 \mathrm{E}+04+1-$ & $7.5 \mathrm{E}+01$ & $1.21:+05+1-$ & $3.618+112$ \\
\hline${ }^{13 s}$ Eu & (Bq/total) & $7.6 \mathrm{E}+06+1-$ & $3.8 \mathrm{E}+05$ & $1.8 \mathrm{E}+04+1$. & $2.0 \mathrm{E}+02$ & $8.9 \mathrm{E}+03+1-$ & $4.5 \mathrm{E}+02$ & $1.51:+05+1$. & $3.0 E+113$ \\
\hline${ }^{219} \mathrm{~Np}$ & (Bq/total) & $4.0 \mathrm{~L}+05+\%$ & $2.0 \mathrm{E}+04$ & $2.1 E+03+1$ & $2,0 \mathrm{E}+02$ & $3.0 \mathrm{E}+03+1-$ & $3.0 \mathrm{E}+02$ & $9.01 i+04+1$. & $9.6 \mathrm{E}+02$ \\
\hline
\end{tabular}


TABLE A2

THE 2nd SET OF OBSERVATIONS (LN SERIES, 28 SAMPLES)

\begin{tabular}{|c|c|c|c|c|c|c|c|}
\hline Analysis & & LN-1 & LN-3 & LN-4 & I.N.6 & LN-7 & LN-8 \\
\hline Gross Alpha & (B) $q /$ lotal) & $1.11 t+06+1-1.9 E+04$ & $2.9 E+06+1-4.4 E+04$ & $1.3 E+06+1-2.1 E+04$ & $2.3 E+06+1 . \quad 3.9 E+04$ & $2.1 \mathrm{E}+05+\% \quad 1.2 \mathrm{E}+04$ & $1.6 \mathrm{E}+06+1-3.2 \mathrm{E}+04$ \\
\hline \multicolumn{8}{|c|}{ Alphor emillers } \\
\hline${ }^{24} \mathrm{Cm}$ & (Bs/totat) & $111:+116+1 . \quad 1.1 E+05$ & $2.81:+06+1 \cdot \quad 2.8 \mathrm{E}+05$ & $1.3 \mathrm{E}+06+1-1.3 \mathrm{E}+05$ & $23 E+06+1 \cdot 2.3 E+0 S$ & $2.0 \mathrm{E}+0 \mathrm{~S}+1.2 .0 \mathrm{E}+04$ & $16 \mathrm{E}+06+1-1.6 \mathrm{E}+05$ \\
\hline${ }^{21 x} \mathrm{Pu}$ & (Bq/total) & $47 \mathrm{E}+02+1.9 .4 \mathrm{E}+01$ & $1.4 \mathrm{E}+03+1 \cdot 2.8 \mathrm{E}+02$ & $9.8 E+02+1-2.0 E+02$ & $1.7 E+03+1-3.4 E+02$ & $3.4 E+03+1-6.8 E+02$ & $7.9 E+02+1-\quad 1.6 E+02$ \\
\hline 211/24" Pu & (Bq/lotal) & $511:+02+1-\quad 1.0 E+02$ & $6.3 \mathrm{E}+03+1-1.3 \mathrm{E}+03$ & $4.0 \mathrm{E}+03+1.8 .0 \mathrm{E}+02$ & $6.3 \mathrm{E}+03+1-\quad 1.3 \mathrm{E}+03$ & $9.5 E+02+1-1.9 \mathrm{~F}+02$ & $4.7 E+03+1-9.4 E+02$ \\
\hline${ }^{212} \mathrm{Pu}$ & (Bq/total) & $201:+01+1-\quad 4.0 E+00$ & $1.5 E:+0 \mid+1-3.1 E+00$ & $20 E+01+1-40 E+00$ & $1.6 \mathrm{E}+01+1-3.2 \mathrm{E}+00$ & $4.8 \mathrm{E}+01+1-9.7 \mathrm{E}+00$ & $4.4 E+01+1-8.8 E+00$ \\
\hline${ }^{211} \mathrm{Am}$ & (Bq/(otal) & $731:+03+1.9 .5 E+01$ & $25 E+03+1-5.4 E+01$ & $22 t:+03+1-52 E+01$ & $7.1 E+03+1-8.1 E+01$ & $2.5 E+03+1.4 .7 E+01$ & $3.2 E+03+1=5.3 E+01$ \\
\hline "'Am & (13q/total) & 4 Sl: $+02+1 . \quad 2.9 E+01$ & $5.7 E+02+1.2 .4 E+01$ & $4.1 E:+02+1-2.5 E+01$ & $6.8 E+02+1.3 .|E+0|$ & $8.0 \mathrm{E}+01+1-1.0 \mathrm{E}+01$ & $3.7 E+02+1-2.2 E+01$ \\
\hline${ }^{212} \mathrm{Cr}$ & (Bq/total) & $161:+04+1-3.3 E+03$ & $2.6 \mathrm{E}+03+1-5.2 \mathrm{E}+02$ & $2.5 \mathrm{E}+03+1 . \quad 4.9 \mathrm{E}+02$ & $3.2 E+03+1 . \quad 6.4 E+02$ & $4.8 E+03+1.9 .7 E+02$ & $28 E+03+1-5.7 E+02$ \\
\hline${ }^{213} \mathrm{Cm}$ & (Buftotal) & $881:+03+\% \quad 1.8 E+03$ & $3.1 E+03+1-6.2 E+02$ & $27 F+03+\%-5.5 F+02$ & $3.7 \mathrm{E}+03+1 . \quad 7.4 \mathrm{E}+02$ & $3.8 \mathrm{E}+03+1 . \quad 7.6 \mathrm{E}+02$ & $3.6 E+03+1-7.1 E+02$ \\
\hline${ }^{211} \mathrm{Cm}$ & (Bu/total) & $<\mid 11:+04$ & $<29 \mathrm{E}+04$ & $<1.3 \mathrm{E}+04$ & $<2.3 E+104$ & $<2.1 \mathrm{E}+03$ & $<1.6 \mathrm{E}+04$ \\
\hline \multicolumn{8}{|c|}{ Beta/gumma emiliers } \\
\hline "'l Co & (Bq//olal) & $421:+(12+1-30 E+(1) 1$ & $481:+0 \mid+1-12 E+01$ & $97 \mathrm{E}+0|+1-| 2 \mathrm{E}+0 \mid$ & $82 \mathrm{E}+101+1 \cdot 1.6 \mathrm{E}+01$ & $<3.3 \mathrm{E}+01$ & $1 \mathrm{SE}+02+1-\quad 1.6 \mathrm{E}+01$ \\
\hline${ }^{\text {Itwo }} \mathrm{Ru}$ & (Bq/(otal) & $56 L+04+1-\quad 7.1 F+02$ & $2.2 E+03+\% \quad 27 E+02$ & $6.6 E+03+1.26 \mathrm{E}+02$ & $1.9 E+(03+1 . \quad 3.3 E+02$ & $8.8 E+02+1-1.9 E+02$ & $1.8 E+03+1-2.3 E+02$ \\
\hline${ }^{111+1+1} \mathrm{AB}$ & (Bq/lotal) & $221:+03+1-3.7 E+01$ & $<54 \mathbf{E}+01$ & $1.2 \mathrm{E}+02+1-15 \mathrm{E}+01$ & $<7.8 \mathrm{E}+01$ & $1.5 E+02+1 . \quad 1.2 \mathrm{E}+01$ & $<5.1 E+01$ \\
\hline "11" Sb & (By/lotal) & $171:+03+1 . \quad 1.5 E+02$ & $25 \mathrm{E}+03+1 \cdot \quad 7.1 \mathrm{E}+01$ & $<1.7 E+02$ & $1.7 E+03+1-18 E+02$ & $<1.5 E+02$ & $1.1 E+03+1-\quad 7.3 E+01$ \\
\hline${ }^{11} \mathrm{Cs}$ & (IBy/lotal) & $171:+(13+1-36 E+01$ & $60 \mathrm{OE}+02+1-20 \mathrm{E}+01$ & $33 \mathrm{E}+02+1 . \quad 1.9 \mathrm{E}+01$ & $2.9 E+03+1 . \quad 3.6 E+01$ & $<3.9 \mathrm{E}+01$ & $7.3 E+02+1 . \quad 2.1 E+01$ \\
\hline${ }^{117} \mathrm{Cs}$ & (Bsq//otal) & $2011:+04+1 . \quad 1.3 E+(12$ & $8.8 t i+03+1.8 .2 E+01$ & $3.6 \mathrm{E}+03+1 . \quad 53 \mathrm{~F}:+01$ & $18 E+04+1 . \quad 1.9 E+02$ & $8.2 \mathrm{E}+02+1-2.9 \mathrm{E}+01$ & $1.1 E+04+1-9.4 E+01$ \\
\hline$" \mathrm{Ce}$ & $\left(\left|B_{1} /(0) \mathrm{n}\right|\right)$ & 901:1(1) +1- $\quad 255:+112$ & $141:+03+1-\quad 1.21 ;+02$ & $1.51 ;+03+1-111 ;+02$ & $2.0 \mathrm{E}+11.3+1-1.6 \mathrm{E}+02$ & $7.1 \mathrm{E}+02+1-8.6 \mathrm{E}+01$ & $15 E+03+1 . \quad 1.0 E+02$ \\
\hline "liu & (By/lotial) & $(101:+1) 2+1-\quad 3.31:+1) 2$ & $<231:+02$ & $101:+02+1-3.21:+01$ & $901:+02 \quad 1 \% \quad 2.01:+02$ & $421:+02+1-7.9 k+1) 1$ & $<261:+112$ \\
\hline "Eu & (|3y/lotal) & $451:+(1) 3+1-\quad 6.81:+111$ & $3.8 E+03+1 . \quad 3.8 E+01$ & $1.9 E+03+1-36 E+01$ & $3.6 \mathrm{E}+(13+1.4 .8 \mathrm{E}+01$ & $15 E+03+1-2.8 E+01$ & $1.8 E+03+1-2.9 E+01$ \\
\hline 19" li:u & (Bu/lotal) & $25 t:+03+1-8.75:+11)$ & $161:+03+1 . \quad 5.6 E+01$ & $9.0 E+02+1.5 .5 E+01$ & $1.9 \mathrm{E}+03+1 . \quad 6.6 \mathrm{E}+01$ & $9.5 E+02+1-4.2 E+01$ & $1.1 E+03+1-4.8 E+01$ \\
\hline${ }^{2 " N} \mathrm{~Np}$ & (Bu/total) & $72 t:+02+1-9.1 E+0 \mid$ & $15 E+03+1-6.3 E+01$ & $8.4 E+02+1.9 .4 E+01$ & $1.2 E+03+1-1.0 E+02$ & $<1.7 \mathrm{E}+02$ & $9.5 E+02+1-\quad 5.1 E+01$ \\
\hline
\end{tabular}




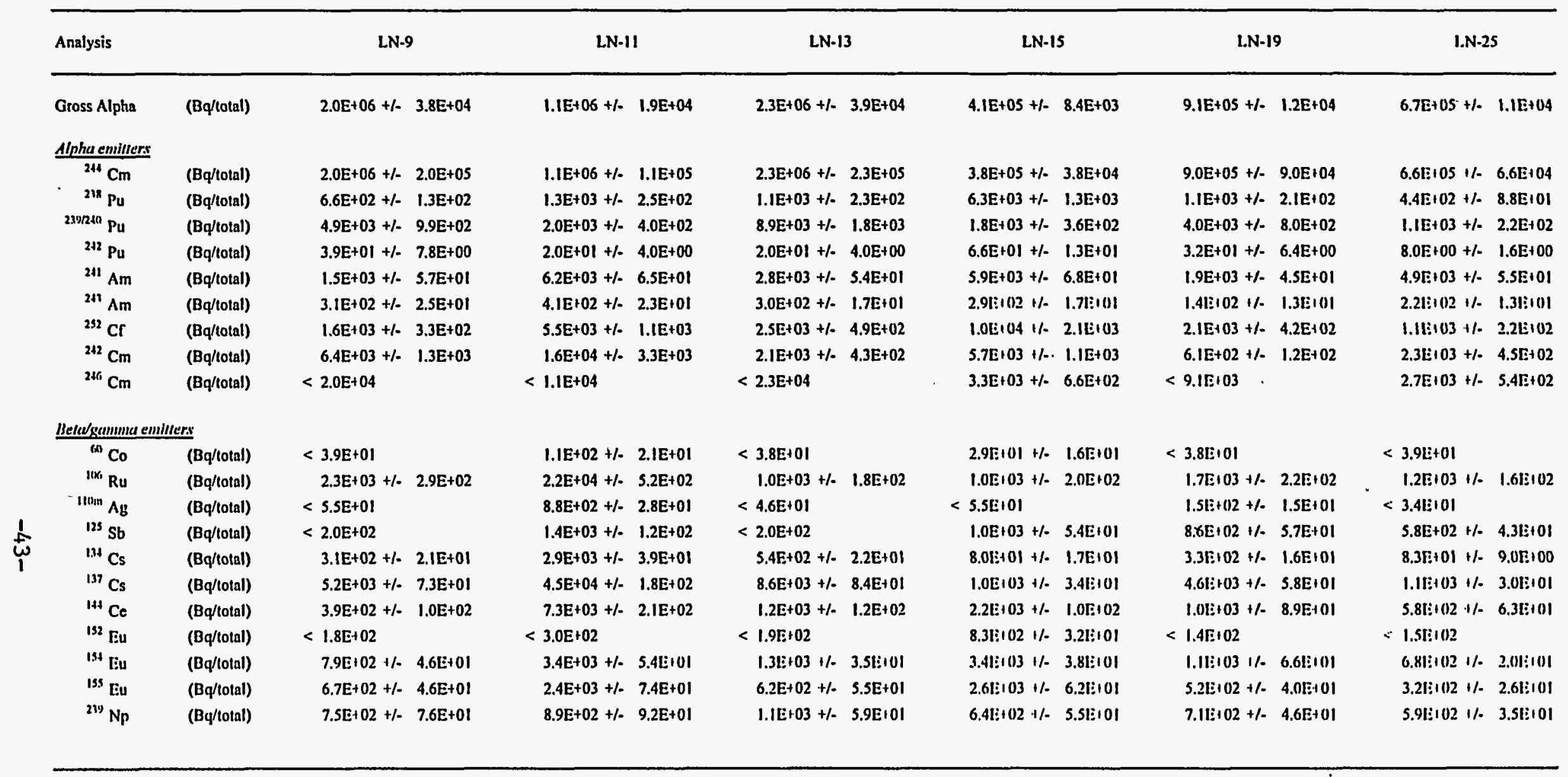




\begin{tabular}{|c|c|c|c|c|c|c|c|}
\hline Analysis & & LN -26 & LN-28 & LN-33 & I.N-35 & I.N-36 & I.N-38 \\
\hline Gross Alpha & (Bq/total) & $1.5 \mathrm{E}+06+1 . \quad 2.2 \mathrm{E}+04$ & $1.1 \mathrm{E}+06+1-1.9 \mathrm{E}+04$ & $1.4 \mathrm{E}+06+1-2.2 \mathrm{E}+04$ & $1.5 \mathrm{E}+06+1-2.2 \mathrm{E}+04$ & $9.5 \mathrm{E}+06+/-7.9 \mathrm{E}+04$ & $2.1 \mathrm{E}+05+/-5.9 \mathrm{E}+03$ \\
\hline \multicolumn{8}{|c|}{ Alpha emitrers } \\
\hline $241 \mathrm{Cm}$ & (Bq//otal) & $1.4 \mathrm{E}+06+1-1.4 \mathrm{E}+05$ & $1.1 E+06+1-1.1 E+05$ & $1.4 \mathrm{E}+06+1-1.4 \mathrm{E}+05$ & $1.4 \mathrm{E}+06+1-1.4 \mathrm{E}+05$ & $9.4 \mathrm{E}+06+1-9.41 \mathrm{E}+05$ & $2.01:+05+1-2.01:+04$ \\
\hline${ }^{21 x} \mathrm{Pu}$ & (Bq/total) & $1.1 \mathrm{E}+03+1-2.2 \mathrm{E}+02$ & $1.4 \mathrm{E}+03+/ \cdot 2.9 \mathrm{E}+02$ & $6.4 \mathrm{E}+03+1-1.3 \mathrm{E}+03$ & $1.4 \mathrm{E}+04+1 \cdot 2.9 \mathrm{E}+03$ & $2.3 \mathrm{E}+03+1 . \quad 4.6 \mathrm{E}+02$ & $6.95+(12+/-1.415+02$ \\
\hline${ }^{2192+11} \mathrm{Pu}$ & (Bqq/otal) & $6.9 \mathrm{E}+03+/ \cdot \quad 1.4 \mathrm{E}+03$ & $6.6 \mathrm{E}+03+/-\quad 1.31:+03$ & $3.6 \mathrm{E}: 03+1-7.2 \mathrm{E}+(12$ & $5.51: 103+1-1.11:+103$ & $3.8 \mathrm{E}+03+1-7.6 \mathrm{E}:+02$ & $4.81 i+(1) 2+1-9.71:+() 1$ \\
\hline${ }^{242} \mathrm{Pu}$ & (Bq/total) & $6.5 \mathrm{E}+01+1-1.3 \mathrm{E}+01$ & $4.9 \mathrm{E}+01+1-9.7 \mathrm{E}+00$ & $5.0 \mathrm{E}+01+1-\quad 1.0 \mathrm{E}+01$ & $2.0 \mathrm{E}:+02+1-4.0 \mathrm{E}+0 \mathrm{I}$ & $2.4 \mathrm{E}+01+\% \quad 4.9 \mathrm{E}+00$ & $2.81:+01+/-5.51:+00$ \\
\hline${ }^{211} \mathrm{Am}$ & (Bq/total) & $1.7 \mathrm{E}+03+1-5.4 \mathrm{E}+01$ & $2.2 \mathrm{E}+03+/-4.9 \mathrm{E}+01$ & $1.5 \mathrm{E}+04+1-9.3 \mathrm{E}+01$ & $5.8 \mathrm{E}+04+1 \cdot 2.0 \mathrm{E}+02$ & $8.3 \mathrm{E}+04+1 \cdot 2.4 \mathrm{E}+02$ & $1.71:+03+1-4.11:+01$ \\
\hline 2.1. Am & (Bq/total) & $2.4 \mathrm{E}+02+/ 2.0 \mathrm{E}+01$ & $2.9 \mathrm{E}+02+1-1.4 \mathrm{E}+01$ & $1.3 E+03+1-3.1 E+01$ & $3.7 \mathrm{E}+02+1-2.7 \mathrm{I}+01$ & $1.0 \mathrm{E}+03+/ 2.9 \mathrm{E}+0\}$ & $1.7 \mathrm{E}+02+1 \cdot \quad 1.7 \mathrm{E}+01$ \\
\hline${ }^{252} \mathrm{Cf}$ & (Bq/total) & $5.8 \mathrm{E}+03+/-\quad 1.2 \mathrm{E}+03$ & $2.1 E+03+/-4.2 E+02$ & $1.3 E+04+1-2.6 E+03$ & $2.1 \mathrm{E}+04+1-4.2 \mathrm{E}+03$ & $4.5 E+03+1-9.0 E+02$ & $1.91:+03+1 . \quad 3.8 \mathrm{E}+02$ \\
\hline${ }^{212} \mathrm{Cm}$ & (Bq/total) & $5.8 \mathrm{E}+03+1-1.2 \mathrm{E}+03$ & $1.2 \mathrm{E}+03+1-2.4 \mathrm{E}+02$ & $9.4 \mathrm{E}+03+1-1.9 \mathrm{E}+03$ & $1.9 \mathrm{E}+04+1-3.9 \mathrm{E}+03$ & $5.0 \mathrm{E}+03+/-1.0 \mathrm{E}+03$ & $1.5 \mathrm{E}:+03+/-2.9 \mathrm{I}:+02$ \\
\hline${ }^{216} \mathrm{Cm}$ & (Bq/total) & $<1.5 \mathrm{E}+04$ & $<1.1 \mathrm{E}+04$ & $<1.4 \mathrm{E}+04$ & $<1.5 E+04$ & $2.9 \mathrm{E}+04+1-5.7 \mathrm{E}+03$ & $8.4 E+02+/-1.7 E+02$ \\
\hline \multicolumn{8}{|c|}{ Beta/gamma emitters } \\
\hline${ }^{(1)} \mathrm{Co}$ & (Bq/total) & $1.9 \mathrm{E}+02+/-2.0 \mathrm{E}+01$ & $2.1 \mathrm{E}+01+/-1.2 \mathrm{E}+01$ & $2.1 \mathrm{E}+02+1-2.4 \mathrm{E}+01$ & $1.71:-1(12+1-2.21:+01$ & $<4.65+01$ & $9.51:+01+1-1.31:+01$ \\
\hline${ }^{H} K_{0} \mathbf{R u}$ & (Bq/total) & $2.1 \mathrm{E}+04+1-4.8 \mathrm{E}+02$ & $2.3 E+03+1-2.9 E+02$ & $2.7 \mathrm{E}+04+1-\quad 6.2 \mathrm{E}+02$ & $4.11:+103+1-4.11:+02$ & $1.31:+03+/-2.01: 402$ & $2.61:+(1) 3+/-2.61:+02$ \\
\hline$\| 111+1 \mathrm{Ag}$ & (Bq/total) & $3.3 \mathrm{E}+03+1-4.0 \mathrm{E}+01$ & $<5.2 \mathrm{E}+01$ & $3.7 \mathrm{E}+02+1-3.0 \mathrm{E}+01$ & $3.5 \mathrm{E}+02+1-2.8 \mathrm{E}+01$ & $<5.4 E+01$ & $1.4 \llbracket:+02+/-\quad 1.41:+01$ \\
\hline${ }^{123} \mathrm{Sb}$ & (Bq/total) & $1.5 \mathrm{E}+03+1-8.3 \mathrm{E}+01$ & $9.9 \mathrm{E}+02+1-6.3 \mathrm{E}+01$ & $3.2 \mathrm{E}+03+1-1.2 \mathrm{E}+02$ & $2.91:+03+1-9.91:+01$ & $1.2 \mathrm{E}+03+1-6.4 \mathrm{E}+01$ & $6.6 \mathrm{E}+02+1 /-4.91 \mathrm{i}+01$ \\
\hline${ }^{134} \mathrm{Cs}$ & (Bq/total) & $5.5 \mathrm{E}+02+1-2.9 \mathrm{E}+01$ & $4.6 \mathrm{E}+02+1-1.9 \mathrm{E}+01$ & $7.1 \mathrm{E}+02+1-\quad 3.7 \mathrm{E}+01$ & $33 \mathrm{l}:+02+/-\quad 3.4 \mathrm{l}:+01$ & $4.1 \mathrm{E}+02+/-2.0 \mathrm{E}+01$ & $2.3 \mathrm{E} \mathrm{E}+02+1-1.5 \mathrm{~L} \mathrm{E}+01$ \\
\hline${ }^{1.177} \mathrm{Cs}$ & (Bq/total) & $3.5 \mathrm{E}+03+1-6.4 \mathrm{E}+01$ & $6.9 E+03+1-7.6 E+01$ & $7.8 E+03+/-8.5 E+01$ & $3.8 \mathrm{E}+03+1-6.6 \mathrm{E}+01$ & $6.3 \mathrm{E}+03+1-6.8 \mathrm{E}+01$ & $1.91 \mathrm{2}+03+1-4.01:+01$ \\
\hline${ }^{\text {IH }} \mathrm{Ce}$ & (Bq/lotal) & $8.6 \mathrm{E}+03+1-1.9 \mathrm{E}+02$ & $1.1 \mathrm{E}+03+1-1.1 \mathrm{E}+02$ & $1.0 \mathrm{E}+04+1-2.3 \mathrm{E}+02$ & $6.0 \mathrm{E}+113+1-1.9 \mathrm{E}:+12$ & $1.1 \mathrm{E}+03+/ \cdot \quad 1.3 \mathrm{E}+02$ & $1.6 \mathrm{t}:+03+1-9.6[\mathrm{E}+01$ \\
\hline 152 l:u & (Bg/total) & $<1.81:+02$ & $<2.1 \mathrm{E}+02$ & $1.51:+03+1-3.01:+102$ & $1.31: 103+1-2.61: 102$ & $<2.11:+02$ & $<2.31:-112$ \\
\hline "st Eu & (Bq/total) & $1.0 E+03+1-4.1 E+01$ & $1.3 \mathrm{E}+03+1-2.5 \mathrm{E}+01$ & $1.7 \mathrm{E}+04+1 \cdot 9.7 \mathrm{E}+01$ & $8.91:+03+/-6.71 \vdots+01$ & $1.61:+03+1-3.51:+01$ & $2.0 \mathrm{E}+03+1-3.11:+01$ \\
\hline${ }^{13 s}$ Eu & (Bq/lotal) & $6.4 \Gamma+02+1-5.9 E+01$ & $7.0 \mathrm{E}+02+1-4.6 \mathrm{E}+01$ & $9.6 \mathrm{E}+03+1 \cdot 1.2 \mathrm{E}+02$ & $8.31:+03+1-1.11:+02$ & $7.6 \mathrm{E}+02+1-5.8 \mathrm{E}+01$ & $1.01:+03+1-3.81:+01$ \\
\hline${ }^{2.19} \mathrm{~Np}$ & (Bq/total) & $1.4 E+03+1-8.8 E+01$ & $7.0 \mathrm{E}+02+1-8.5 \mathrm{E}+01$ & $2.1 E+03+1 \cdot \quad 1.2 E+02$ & $1.41 \mathrm{E}+03+1-1.2 \mathrm{~L}+02$ & $4.8 \mathrm{E}+03+/ . \quad 1.7 \mathrm{E}+02$ & $2.4 E+02+/-3.315+01$ \\
\hline
\end{tabular}




\begin{tabular}{|c|c|c|c|c|c|c|c|}
\hline Analysis & & LN-39 & $1 . N-40$ & $|. N-4|$ & I.N-44 & I.N-46 & $1 . N-48$ \\
\hline Gross Alpha & (Bq/total) & $1.3 \mathrm{E}+07+1-1.3 \mathrm{E}+05$ & $1.4 \mathrm{E}+06+1-2.2 \mathrm{E}+04$ & $5.5 E+05+1-9.7 E+03$ & $3.5 \mathrm{E}+05+1-7.6 \mathrm{E}+03$ & $1.9 \mathrm{E}+05+1-1.6 \mathrm{E}+03$ & $3.9 E+05+1-1.0 E+04$ \\
\hline \multicolumn{8}{|l|}{$\frac{\text { Alpha enilters }}{2 s t}$} \\
\hline${ }^{244} \mathrm{Cm}$ & (Bq/total) & $1.3 \mathrm{E}+07+1-\quad 1.3 \mathrm{E}+06$ & $1.4 \mathrm{E}+06+1-\quad 1.4 \mathrm{E}+05$ & $5.3 E+05+1-5.3 E+04$ & $3.4 E+05+1-3.4 E+04$ & $1.9 \mathrm{E}+05+1-\quad 1.9 \mathrm{I}+04$ & $3.8 E+05+/-3.8 E+04$ \\
\hline${ }^{21 x} \mathrm{Pu}$ & (Bq/total) & $3.0 \mathrm{E}+03+1-6.1 \mathrm{E}+02$ & $3.8 \mathrm{E}+02+1-7.5 \mathrm{E}+01$ & $2.0 \mathrm{E}+03+1-3.9 \mathrm{E}+02$ & $3.1 E+03+1.6 .3 E+02$ & $1.1 \mathrm{E}+03+1-2.2 \mathrm{E}+02$ & $1.6 \mathrm{E}+03+/-3.1 \mathrm{E}+02$ \\
\hline${ }^{2.1912+10} \mathrm{Pu}$ & (Bq/total) & $6.2 E+03+1-1.2 E+03$ & $2.9 \mathrm{E}+02+1-5.7 \mathrm{E}+01$ & $2.1 \mathrm{E}+03+/-4.2 \mathrm{E}+02$ & $1.4 E+03+1-2.9 E+02$ & $6.8 \mathrm{E}+02+1-1.4 \mathrm{E}+02$ & $9.3 \mathrm{E}:+02+/-1.9 \mathrm{E}+02$ \\
\hline${ }^{2+2} \mathrm{Pu}$ & (Bq/total) & $9.3 \mathrm{E}+00+1-1.9 \mathrm{E}+00$ & $8.7 E+00+/-1.7 E+00$ & $3.3 \mathrm{E}+01+1-6.6 \mathrm{E}+00$ & $4.6 \mathrm{E}+01+1-9.2 \mathrm{E}+00$ & $1.7 \mathrm{E}+01+1-3.4 \mathrm{E}+00$ & $1.3 \mathrm{E} E+01+1-2.5 E+00$ \\
\hline $241 \mathrm{Am}$ & (Bq/total) & $1.1 E+05+/-2.7 E+02$ & $1.1 E+04+1.5 .9 E+02$ & $7.2 \mathrm{E}+03+\% \quad 7.7 \mathrm{E}+01$ & $2.2 E+03+1-4.3 E+01$ & $2.0 \mathrm{E}+03+1-4.2 \mathrm{E}+01$ & $3.7 \mathrm{~F}:+03+1-5.6 \mathrm{l}:+01$ \\
\hline${ }^{241}$ Am & (Bq/total) & $1.2 \mathrm{E}+03+1-3.1 \mathrm{E}+01$ & $5.8 \mathrm{E}+03+1-3.3 \mathrm{E}+02$ & $2.1 \mathrm{E}+02+1-2.0 \mathrm{E}+01$ & $1.9 E+02+1-\quad 1.4 E+01$ & $1.1 \mathrm{E}+02+1-1.6 \mathrm{E}+01$ & $1.5 i \mathfrak{i}+02+1-1.3 \hat{E}+01$ \\
\hline${ }^{232} \mathrm{Cf}$ & (Bq/total) & $5.2 \mathrm{E}+03+1-1.0 \mathrm{E}+03$ & $1.8 \mathrm{E}+04+1-3.5 \mathrm{E}+03$ & $6.0 \mathrm{E}+03+1 . \quad 1.2 \mathrm{E}+03$ & $1.25:+03+1-2.4[E+02$ & $1.8 \mathrm{E}+03+/-3.5 \mathrm{E}+02$ & $2.5 E+03+1 . \quad 4.9 E++02$ \\
\hline${ }^{242} \mathrm{Cm}$ & (Bq/total) & $7.8 \mathrm{E}+03+1-1.6 \mathrm{E}+03$ & $1.3 \mathrm{E}+04+1-2.6 \mathrm{E}+03$ & $1.7 \mathrm{E}+03+1-3.4 \mathrm{E}+02$ & $4.11:+1) 3+1-8.15:+02$ & $3.2 \mathrm{E}+02+1-6.5 \mathrm{E}+01$ & $1.41:+03+1-2.91:+02$ \\
\hline${ }^{2+11} \mathrm{Cm}$ & (Bq/total) & $3.9 \mathrm{E}+04+/-7.8 \mathrm{E}+03$ & $<1.4 \mathrm{E}+04$ & $4.8 E+03+1-9.6 E+02$ & $<3.51:+103$ & $3.8 \mathrm{I}:+02+1-7.6 \mathrm{E}+01$ & $<3.91:+03$ \\
\hline \multicolumn{8}{|c|}{$\frac{\text { Beta/gamma entitters }}{(1)}$} \\
\hline${ }^{(1)} \mathrm{Co}_{0}$ & (Bq/total) & $5.0 \mathrm{E}+01+1-1.2 \mathrm{E}+01$ & $<3.7 \mathrm{E}+02$ & $1.4 \mathrm{E}+02+1-1.4 \mathrm{E}+01$ & $3.91 j+01+1 . \quad 1.11 i+01$ & $1.1 E+02+1-1.4 E+01$ & $6.7 E+(01+1-1.118+01$ \\
\hline${ }^{\mathrm{H} K_{\mathrm{s}}} \mathrm{Ru}$ & (Bq/total) & $2.2 \mathrm{E}+03+1-2.1 \mathrm{E}+02$ & $6.2 \mathrm{E}+05+/-\quad 6.7 \mathrm{E}+03$ & $7.3 \mathrm{E}+03+1-3.4 \mathrm{E}+02$ & $7.8 \mathrm{E}+102+1-1.35:+12$ & $3.3 \mathrm{k}+03+/-2.1 E+02$ & $3.0 \mathrm{E}+03+/-2.8 \mathrm{~F}:+01$ \\
\hline$\| 111111 \mathrm{Ag}$ & (Bq/total) & $2.0 \mathrm{E}+02+1-1.8 \mathrm{E}+01$ & $3.1 \mathrm{E}+03+/-2.6 \mathrm{E}+02$ & $1.6 \mathrm{E}+02+1 . \quad 1.6 \mathrm{E}+01$ & $2.61:+02+1-\quad 1.31:+01$ & $<4.8 \mathrm{~F}+01$ & $<4.61:+01$ \\
\hline${ }^{125} \mathrm{Sb}$ & (Bq/total) & $1.4 \mathrm{E}+03+1-7.1 \mathrm{E}+01$ & $<3.0 \mathrm{E}+03$ & $3.9 E+03+1-1.1 E+02$ & $5.11:+(1) 2+1 \cdot 3.91 i+(1) 1$ & $53 E+02+1-4.1 E++01$ & $8.91:+102+1-6.31:+1) 1$ \\
\hline${ }^{134} \mathrm{Cs}$ & (Bq/total) & $3.0 \mathrm{E}+02+1-2.0 \mathrm{E}+01$ & $<1.2 \mathrm{E}+04$ & $4.9 \mathrm{E}+02+1-3.1 \mathrm{E}+01$ & $261:+02+1-1.21:+01$ & $2.5 E+02+/-1.4 E+01$ & $3.51:+02+1-2.25:+01$ \\
\hline $17 \mathrm{Cs}$ & (Bq/total) & $4.4 \mathrm{E}+03+1-5.9 \mathrm{E}+01$ & $1.7 \mathrm{E}+04+1-5.2 \mathrm{E}+02$ & $6.3 E+03+1-6.9 E+01$ & $3.91:+103+1 \cdot 5.51:+01$ & $2.2 \mathrm{E}+03+/-4.2 \mathrm{E}+01$ & $4.6 \mathrm{E}+03+1.6 .41:+01$ \\
\hline H+ Ce & (Bq/10tal) & $7.0 \mathrm{E}+02+1-8.6 \mathrm{E}+01$ & $1.9 \mathrm{E}+04+1-1.8 \mathrm{E}+03$ & $1.2 E+03+1-9.9 E+01$ & $1.015+103+1-8.71:+01$ & $1.21:+03+1-8.1 t i+01$ & $1.31:+103+1-9.81:+01$ \\
\hline 192 Eu & (Bq/total) & $<2.2 \mathrm{E}+02$ & $<1.5 \mathrm{E}+03$ & $<2.1 \mathrm{t}:+02$ & $<1.71:+112$ & $<1.81:+02$ & $<2.01 \vdots+02$ \\
\hline Ist Liu & (Bq/lotal) & $1.5 \mathrm{E}+03+1-3.6 \mathrm{E}+01$ & $4.1 E+04+/-1.4 E+03$ & $2.81 E+03+1-4.2 E+01$ & $6.011++02+1 /-1.71:+01$ & $1.41:+03+1-2.91:+01$ & $2.11:+113+1-3.51:+1111$ \\
\hline${ }^{1.95}$ Eu & (Bq/lotal) & $3.5 E+02+1-3.9 E+01$ & $1.7 \mathrm{E}+04+1-7.3 \mathrm{E}+02$ & $6.8 \mathrm{E}+02+/ . \quad 4.1 \mathrm{E}+01$ & $3.21:+02+1-3.21:+01$ & $1.0 E+03+1 . \quad 4.2 E+01$ & $1.01:+03+1-5.3 \mid z+0 t$ \\
\hline${ }^{219} \mathrm{~Np}$ & (Bq/total) & $3.5 \mathrm{E}+03+1-1.2 \mathrm{E}+03$ & $<4.3 \mathrm{E}+03$ & $<2.7 \mathrm{E}+02$ & $3.61:+102+1-3.5 E+01$ & $2.6 \mathrm{E}+02+/-4.0 \mathrm{E}+01$ & $4.51:+02+1.5 .118+01$ \\
\hline
\end{tabular}




\begin{tabular}{|c|c|c|c|c|c|c|c|c|c|}
\hline \multirow{2}{*}{$\begin{array}{l}\text { Analysis } \\
\text { Gross Alpha }\end{array}$} & \multirow[b]{2}{*}{ (Bq/total) } & \multicolumn{2}{|c|}{ I.N-49 } & \multicolumn{2}{|c|}{ LN-S4 } & \multicolumn{2}{|c|}{ LN-GL } & \multicolumn{2}{|c|}{ LN-ML. } \\
\hline & & $1.2 \mathrm{E}+05+1-$ & $4.4 \mathrm{E}+03$ & $6.0 \mathrm{E}+06+1-$ & $1.0 \mathrm{E}+04$ & $1.2 \mathrm{E}+08+1-$ & $1.7 \mathrm{E}+06$ & $7.3 \mathrm{~F}+07+1$. & $1.415+116$ \\
\hline \multicolumn{10}{|l|}{ Alpha emilters } \\
\hline${ }^{244} \mathrm{Cm}$ & (Bq/1otal) & $1.2 \mathrm{E}+05+1$. & $1.2 \mathrm{E}+04$ & $5.9 \mathrm{E}+05+1-$ & $5.9 \mathrm{E}+04$ & $1.2 \mathrm{E}+08+\%$ & $1.2 \mathrm{E}+07$ & $6.9 E+07+1-$ & $6.9 t+06$ \\
\hline${ }^{2.13} \mathrm{Pu}$ & (Bq/total) & $4.0 \mathrm{E}+02+/ \%$ & $8.0 \mathrm{E}+01$ & $1.4 \mathrm{E}+03+1$. & $2.8 \mathrm{E}+02$ & $2.7 E+05+\%$ & $5.5 E+04$ & $1.1 E+06+1$ & $2.2 \mathrm{E}+05$ \\
\hline 211/2+11 Pu & (Bq/total) & $4.0 \mathrm{E}+02+1-$ & $8.0 E+01$ & $1.1 E+03+1-$ & $2.3 \mathrm{E}+02$ & $3.2 \mathrm{E}+05+1-$ & $6.4 \mathrm{E}+04$ & $2.9 E+05+1$ & $5.8 E+04$ \\
\hline${ }^{242} \mathrm{Pu}$ & (Bq/total) & $<8.2 \mathrm{E}+01$ & & $3.4 \mathrm{E}+01+1-$ & $6.8 \mathrm{E}+00$ & $4.2 \mathrm{E}+03+1$ & $8.4 \mathrm{E}+02$ & $1.3 k+04+1-$ & $2.5[:+03$ \\
\hline${ }^{2+1} \wedge \mathrm{m}$ & (Bq/total) & $8.9 E+02+1 /$ & $2.8 \mathrm{E}+01$ & $52 E+03+1$ & $5.98:+01$ & $6.2 \mathrm{E}+05+1-$ & $66 \mathrm{~F}:+03$ & $9.31:+105+1$. & $5.51:+03$ \\
\hline${ }^{241} \mathrm{Aln}$ & (Bq/(otal) & $8.81:+01+1$. & $8.4 \mathrm{E}+\infty 0$ & $3.2 \mathrm{E}+02+1$. & $1.51 ;+01$ & $711:+04+1-$ & $181:+113$ & $491:+104+1-$ & $1.11: 103$ \\
\hline${ }^{2.22} \mathrm{Cr}$ & (Bq/total) & $7.8 \mathrm{E}+02+1-$ & $1.6 \mathrm{E}+02$ & $2.5 \mathrm{E}+03+1$ & $4.9 \mathrm{E}+02$ & $1.3 \mathrm{E}+06+1-$ & $2.6 \mathrm{E}+05$ & $2.0 L+06+1-$ & 4.01:+05 \\
\hline${ }^{212} \mathrm{Cm}$ & (Bq/total) & $3.0 \mathrm{E}+02+1$. & $6.0 \mathrm{E}+01$ & $2.3 E+03+1-$ & $4.7 \mathrm{E}+02$ & $S .0 \mathrm{E}+0 \mathrm{~S}+1$. & $1.0 \mathrm{n}+0 \mathrm{~s}$ & $4.115+05+1-$ & $8.1 \mathrm{E}+04$ \\
\hline${ }^{2316} \mathrm{Cm}$ & (Bq/total) & $<1.2 \mathrm{E}+03$ & & $1.8 \mathrm{E}+03+1$. & $3.6 \mathrm{E}+02$ & $<1.2 \mathrm{E}+06$ & & $<7.31:+05$ & \\
\hline \multicolumn{10}{|c|}{ Beta/gamma emitters } \\
\hline${ }^{(1)} \mathrm{Co}$ & (Bq/total) & $3.8 \mathrm{E}+01+\%$ & $1.0 \mathrm{E}+01$ & $7.0 \mathrm{E}+01+1-$ & $1.3 \mathrm{E}+01$ & $4.7 E+04+1-$ & $1.61:+03$ & $6.51:+03+1-$ & $1.08:+103$ \\
\hline${ }^{\prime \mid X_{1}}$ Ru & (Bq/total) & $1.1 \mathrm{E}+03+1$. & $1.5 \mathrm{E}+02$ & $2.6 \mathrm{E}+03+/-$ & $2.2 \mathrm{E}+02$ & $5.2 \mathrm{E}+06+1-$ & $4.6 \mathrm{i}+04$ & $3.7 E+05+1$ & $2.2 \mathrm{E}+(1) 4$ \\
\hline${ }^{1110+1} \mathrm{Ag}$ & (Bq/total) & $<3.1 E+01$ & & $1.5 \mathrm{E}+02+1$ & $1.3 \mathrm{E}+01$ & $7.78+04+1$ & $2.1 \mathrm{E}+03$ & $1.7 \mathrm{i}+104+1-$ & $1.11:+03$ \\
\hline${ }^{123} \mathrm{Sb}$ & (Bq/lotal) & $2.7 E+02+1-$ & $3.1 \mathrm{E}+01$ & $5.6 \mathrm{E}+02+1 /$ & $4.7 \mathrm{E}+01$ & $2.3 E+05+1-$ & $3.5 \mathrm{E}+04$ & $1.1 \mathrm{E}+05+1-$ & $4.01:+103$ \\
\hline${ }^{13} \mathrm{Cs}$ & (Bq/total) & $9.1 E+01+1$. & $9.8 \mathrm{E}+00$ & $2.2 \mathrm{E}+02+1-$ & $1.8 \mathrm{E}+01$ & $5.0 \mathrm{E}+05+1-$ & $3.2 \mathrm{E}+03$ & $1.4 k+04+1 /$ & 1.015:+03 \\
\hline${ }^{1317} \mathrm{Cs}$ & (Bq/total) & $8.7 \mathrm{E}+02+1-$ & $3.1 \mathrm{E}+01$ & $3.0 \mathrm{E}+03+1$ & $4.9 \mathrm{E}+01$ & $6.7 \mathrm{E}+06+1-$ & $1.4 \Sigma+04$ & $1.51:+05+1-$ & $2.51:+1) 3$ \\
\hline${ }^{i+1} \mathrm{Ce}$ & (Bq/(otal) & $5.1 \mathrm{E}+02+1$. & $6.0 E+01$ & $2.7 \mathrm{E}+03+1$ & $1.15:+02$ & $5.5 \mathrm{E}+05+1-$ & $1.65:+04$ & $33 E:+05+1-$ & 8.01:+103 \\
\hline${ }^{152}$ Eu & (Bq/otal) & $<1.7 \mathrm{E}+02$ & & $3.2 E+02+1-$ & $1.3 \mathrm{E}+02$ & $5.6 \mathrm{E}+04+1-$ & $3.1 \mathrm{E}+03$ & $1.4 \mathrm{E}+05+1-$ & $2.6 \mathrm{~B}+03$ \\
\hline 194 Eu & (Bq/otal) & $7.8 \mathrm{E}+02+1$. & $1.8 \mathrm{E}+01$ & $2.6 \mathrm{E}+03+1-$ & $3.6 \mathrm{E}+01$ & $3.9 \mathrm{E}+05+1-$ & $3.4 \Sigma+03$ & $5.2 \mathrm{~F}+05+1 /$ & $2.95:+03$ \\
\hline${ }^{13 S}$ Eu & (Bq/otal) & $3.8 \mathrm{E}+02+/$ & $2.9 \mathrm{E}+01$ & $1.3 \mathrm{E}+03+1-$ & $5.0 \mathrm{E}+01$ & $2.4 \mathrm{E}+05+1-$ & $4.6 \mathrm{E}+03$ & $4.4 \mathrm{i}+05+1-$ & $4.4 E+04$ \\
\hline${ }^{219} \mathrm{~Np}$ & (Bq/total) & $1.6 \mathrm{E}+02+1 /$ & $3.0 \mathrm{E}+01$ & $5.2 \mathrm{E}+02+1-$ & $4.2 \mathrm{E}+01$ & $1.5 \mathrm{E}+05+1-$ & $9.61:+03$ & $9.01+04+/-$ & $1.8 E+04$ \\
\hline
\end{tabular}


LN-GL

3 glass bottles weighing 80.28

Analysis

Result Error

$1.2 E+08+\% \quad 1.7 E+06$

Alpha emitters

\begin{tabular}{|c|c|c|c|}
\hline${ }^{24} \mathrm{Cm}$ & (Bq/total) & $1.2 \mathrm{E}+08+/-$ & $1.2 E+07$ \\
\hline${ }^{230} \mathrm{Pu}$ & (Bq/total) & 2.7E $+05+1$ & $5.5 E+04$ \\
\hline${ }^{239200} \mathrm{Pu}$ & (Bq/total) & $3.2 \mathrm{E}+05+1$ & $6.4 E+04$ \\
\hline${ }^{20} \mathrm{Pu}$ & (Bq/total) & $4.2 E+03+1-$ & $8.4 E+02$ \\
\hline 211 Am & (Bq/total) & $6.2 E+05+/-$ & $6.6 E+03$ \\
\hline${ }^{203} \mathrm{Am}$ & (Bq/total) & $7.1 E+04+/-$ & $1.8 E+03$ \\
\hline${ }^{249} \mathrm{Cf}$ & (Bq/total) & $4.0 E+03+/-$ & $8.0 E-02$ \\
\hline${ }^{250} \mathrm{Cf}$ & (Bq/total) & $5.1 E+04+/-$ & $1.0 E+04$ \\
\hline${ }^{251} \mathrm{Cr}$ & (Bq/total) & $2.3 E+02+/-$ & $4.7 E+01$ \\
\hline${ }^{252} \mathrm{Cf}$ & (Bq/total) & $1.3 E+06+/$ & $2.6 E+05$ \\
\hline${ }^{253} \mathrm{Cf}$ & (Bq/total) & $3.5 E+04+1-$ & $7.0 \mathrm{E}+03$ \\
\hline${ }^{234} \mathrm{Cf}$ & (Bq/total) & $6.9 E+03+/-$ & $1.4 E+03$ \\
\hline${ }^{242} \mathrm{Cm}$ & (Bq/total) & $5.0 \mathrm{E}+05+1-$ & $1.0 E+05$ \\
\hline${ }^{245} \mathrm{Cm}$ & (Bq/total) & $4.4 E+03+\%$ & $4.4 E+02$ \\
\hline${ }^{246} \mathrm{Cm}$ & (Bq/total) & $1.7 E+05+1-$ & $1.7 E+04$ \\
\hline${ }^{207} \mathrm{Cm}$ & (Bq/total) & $1.2 \mathrm{E}+00+1-$ & $1.2 \mathrm{E}-01$ \\
\hline${ }^{248} \mathrm{Cm}$ & (Bq/total) & $3.5 E+02+\%$ & $3.5 E+01$ \\
\hline
\end{tabular}

Betalgamma emitters

${ }^{60} \mathrm{Co} \quad$ (Bqhotal)

4.7E+04 +/ $\quad 1.6 \mathrm{E}+03$

${ }^{106} \mathrm{Ru} \quad(\mathrm{Bq} / \mathrm{total})$

$5.2 E+06+1-4.6 E+04$

$110 \mathrm{~m} \mathrm{Ag} \quad(\mathrm{Bq} / \mathrm{total})$

7.7E $+04+\% \quad 2.1 \mathrm{E} \div 03$

$123 \mathrm{Sb}$ (Bq/total)

2.3E+05 +\% 3.5E+04

${ }^{134}$ Cs (Bqhotal)

$5.0 \mathrm{E}+05+1-\quad 3.2 \mathrm{E}+03$

${ }^{137} \mathrm{Cs}$ (Bq/total)

6.7E+06 +\% 1.4E+04

${ }^{14} \mathrm{Ce} \quad(\mathrm{Bq} /$ total)

$5.5 E+05+/-1.6 E+04$

152 Eu (Bq/total)

$5.6 \mathrm{E}+04+\% \quad 3.1 \mathrm{E}-03$

${ }^{154} \mathrm{Eu}$ (Bq/total)

$3.9 E+05+1-3.4 E+03$

${ }^{155} \mathrm{Eu} \quad$ (Bq/total)

${ }^{239} \mathrm{~Np} \quad$ (Bq/total)

Total Alpha Concentration =

$4.0 \mathrm{E}+04 \mathrm{nCi} / \mathrm{g}$

TRU Concentration $=$

$495.7 \mathrm{nCi} / \mathrm{g}$ 
Three glass sample bottles (LN-GL) were removed from a waste can in cubicle 8 in order to leach. The bottles were placed in a beaker filled with $400 \mathrm{~mL}$ of $8 \mathrm{~N}$ Nitric Acid and allowed to leach for approximately four hours. Following the leach an aliquot of the total leachate was taken from the beaker for radiochemical analyses. The mass of the three bottles had to be estimated because there are no current means of weighing inside the cell bank. Three sets of three sample bottles identical to those leached were weighed resulting in an average of 80.2 grams. This mass was combined with the radiochemical analyses to provide a TRU Concentration for the set of bottles. 
TABLE A4

SAMPLE TRACKING RECORD

First data set

\begin{tabular}{|c|c|c|c|c|c|}
\hline $\begin{array}{c}\text { SAMPLE } \\
\text { NUMNBER }\end{array}$ & CELL LOC. & $\begin{array}{l}\text { SAMPLING } \\
\text { DATE }\end{array}$ & $\begin{array}{c}\text { WASTE } \\
\text { CATEGORY }\end{array}$ & WASTE ITEM DESCRIPTION & TYPE OF SAMPLE \\
\hline RH9AA & 9 & $10 / 26 / 95$ & Plastic & Melted Poly & Smear \\
\hline RH9EE & 9 & $10 / 26 / 95$ & Plastic & Poly Valve & Smesr \\
\hline RH9KK & 9 & $10 / 26 / 95$ & Plastic & Bottle lid & Smear \\
\hline RH9L & 9 & $10 / 26 / 95$ & Glass & Sample bottle & Smas \\
\hline RH9OQ & 9 & $10 / 26 / 95$ & Metal & Wire & Smear \\
\hline RH9SS & 9 & $10 / 26 / 95$ & Cloth & Wipes & Smear \\
\hline RH9A & 9 & $1 / 11 / 96$ & Plastic & Melted Poly & Smear \\
\hline RH9C & 9 & $1 / 11 / 96$ & Plastic & Melted Poly & Smear \\
\hline AH9D & 9 & $1 / 11 / 96$ & Plastic & Bain Marie bucket & Smear \\
\hline AH9E & 9 & $1 / 11 / 96$ & Plastic & Social FDV poly valve & Smear \\
\hline RH9F & 9 & $1 / 11 / 96$ & 'Plastic & PVC Valve & Smear \\
\hline RH9G & 9 & $1 / 11 / 96$ & Plastic & PVC Valve & Smear \\
\hline RH9H & 9 & $1 / 11 / 96$ & Plastic & Light cord & Smear \\
\hline BH9I & 9 & $1 / 11 / 96$ & Plastic & MSM boot & Smear \\
\hline RH9J & 9 & $1 / 11 / 96$ & Plastic & Poly bottle lid & Smear \\
\hline RH9K & 9 & $1 / 11 / 96$ & Plastic & Bottle lid & Smear \\
\hline RH9L & 9 & $1 / 10 / 96$ & Glass & Sample bottle & Smear \\
\hline AH9M & 9 & $1 / 11 / 96$ & Glass & Sample bottle & Smear \\
\hline AH9N & 9 & $1 / 11 / 96$ & Glass & Glassware & Smoar \\
\hline AH9O & 9 & $1 / 11 / 96$ & Glass & Filter housing & Smear \\
\hline RH9P & 6 & $1 / 11 / 96$ & Metal & Metal tool & Smear \\
\hline RH9Q & 9 & $1 / 11 / 96$ & Metal & PreAmp lead can & Smear \\
\hline RH9R & 9 & $1 / 11 / 96$ & Metal & Stainless steel lid & Smear \\
\hline RH9S & 9 & $10 / 26 / 95$ & Cloth & Wipes & Smear \\
\hline RH9T & 9 & $1 / 11 / 96$ & Cloth & Wipes & Smear \\
\hline $\mathrm{RH9U}$ & 9 & $1 / 11 / 96$ & Cloth & Wipes & Smear \\
\hline RHgV & 9 & $1 / 11 / 96$ & Cloth & \begin{tabular}{|l|} 
String \\
\end{tabular} & Smear \\
\hline RH1B & 1 & $1 / 11 / 96$ & & MSM Boot & Smear \\
\hline $\mathrm{RH} 2 \mathrm{~B}$ & 2 & $1 / 11 / 96$ & & MSM Boot & Smear \\
\hline $\mathrm{RH} 3 \mathrm{~B}$ & 3 & $1 / 11 / 96$ & & MSM Boot & Smear \\
\hline RH4D & 4 & $1 / 11 / 96$ & & Cubicle Sump & Smear \\
\hline $\mathrm{RH} 6 \mathrm{C}$ & 6 & $1 / 11 / 96$ & & Cubicle Sump & Smear \\
\hline RH7D & 7 & $1 / 11 / 96$ & & Cubicle Sump & Smear \\
\hline RH8A & 8 & $1 / 11 / 96$ & & Cubicle Sump & Smear \\
\hline
\end{tabular}

Total number of samples $=34$ 
TABLE A5

SAMPLE TRACKING RECORD

Second data set

\begin{tabular}{|c|c|c|c|c|c|}
\hline $\begin{array}{c}\text { SAMPLE } \\
\text { NUMNBER }\end{array}$ & CELL LOC. & $\begin{array}{c}\text { SAMPLNG } \\
\text { DATE }\end{array}$ & $\begin{array}{l}\text { WASTE } \\
\text { CATEGORY }\end{array}$ & WASTE ITEM DESCRIPTION & TYPE OF SAMPLE \\
\hline LN-1 & 9 & $5 / 1 / 96$ & glass & bottle & Smear \\
\hline LN-3 & 9 & $5 / 1 / 96$ & glass & bottle & Smear \\
\hline $\mathrm{LN}-4$ & 9 & $5 / 1 / 96$ & glass & bottle & Smear \\
\hline LN-6 & 9 & $5 / 1 / 96$ & glass & bottle & Smear \\
\hline LN-7 & 9 & $5 / 1 / 96$ & metal & tool & Smeer \\
\hline LN-8 & 9 & $5 / 1 / 96$ & glass & bottle & Smear \\
\hline LN-9 & 9 & $5 / 1 / 96$ & glass & bulb & Smear \\
\hline LN-11 & 9 & $5 / 1 / 96$ & glass & bottle & Smear \\
\hline LN-13 & 9 & $5 / 1 / 96$ & glass & bottle & Smear \\
\hline LN-15 & 9 & $5 / 1 / 96$ & metal & tool & Smear \\
\hline LN-19 & 9 & $5 / 1 / 96$ & glass & bottle & Smear \\
\hline LN-25 & 9 & $5 / 1 / 96$ & glass & bottle & Smear \\
\hline LN-26 & 9 & $5 / 1 / 96$ & glass & bottle & Smear \\
\hline LN-28 & 9 & $5 / 1 / 96$ & glass & bottle & Smear \\
\hline LN-33 & 9 & $5 / 1 / 96$ & poly & valve & Smear \\
\hline LN-35 & 9 & $5 / 1 / 96$ & poly & bottle & Smear \\
\hline LN-36 & 9 & $5 / 1 / 96$ & metal & cell & Smear \\
\hline LN-38 & 9 & $5 / 1 / 96$ & metal & tool & Smear \\
\hline LN-39 & 9 & $5 / 1 / 96$ & metal & cell & Smear \\
\hline LN-40 & 9 & $5 / 1 / 96$ & poly & hose & Smear \\
\hline LN-41 & 9 & $5 / 1 / 96$ & metal & column & Smear \\
\hline LN-44 & 9 & $5 / 1 / 96$ & metal & cap & Smear \\
\hline LN-46 & 9 & $5 / 1 / 96$ & metal & can & Smear \\
\hline LN-48 & 9 & $5 / 1 / 96$ & metal & tool & Smear \\
\hline LN-49 & 9 & $5 / 1 / 96$ & metal & socket & Smear \\
\hline LN-54 & 9 & $5 / 1 / 96$ & metal & case & Smear \\
\hline LN-GL & 9 & $5 / 1 / 96$ & glass & bottles & Leach \\
\hline LN-ML & 9 & $5 / 1 / 96$ & metal & tool & Leach \\
\hline
\end{tabular}

Total number of samples $=28$

Notes:

1. LN-ML (Metal leach of a tooll has duplicate smears performed on the tool prior to leaching: $\mathrm{LN}-7$ and $\mathrm{LN}-15$

2. LN-GL (Glass leach of 3 bottles) has duplicate smears performed on the bottles prior to leaching: LN-1 and LN-11

3. LN-19 and LN-28 are duplicate emears of the same glase bottle

4. LN-36 and LN-39 are duplicate emears of a motal woighing call 
Appendix 2. Linear Regression for Poisson-Distributed Data with Special Reference to Spectrometry

This appendix outlines the method used to obtain the individual radionuclide activities from the sample spectrograph using methods based on linear Poisson regression. Let $\mathbf{y}$ be the $n \times 1$ vector of observed counts from the $n$ channels of interest in the spectrograph. If we assume the elements in $y$ to be the realization of $n$ independent Poisson rańdom variables, then the likelihood function of the sample vector $\mathbf{y}$ is given by

$$
L(\mathbf{y}, \boldsymbol{\theta})=\prod_{i=1}^{n} \frac{\exp \left(-\mu\left(x_{i}, \boldsymbol{\theta}\right)\right) \mu\left(x_{i}, \boldsymbol{\theta}\right)^{y_{i}}}{y_{i} !}
$$

or

$$
l(\mathbf{y}, \boldsymbol{\theta})=\log (L(\mathbf{y}, \boldsymbol{\theta}))=-\sum_{i=1}^{n} \mu\left(x_{i}, \boldsymbol{\theta}\right)+\sum_{i=1}^{n} y_{i} \ln \mu\left(x_{i}, \boldsymbol{\theta}\right)+\mathrm{constant}
$$

where

$$
\mu\left(x_{i}, \theta\right)=\theta_{1} \psi_{1}\left(x_{i}\right)+\ldots+\theta_{m} \psi_{m}\left(x_{i}\right)=\theta^{T} \Psi\left(x_{i}\right)
$$

Our immediate objective is to maximize $l(y, \theta)$ with respect to $\theta$. It should be noted that in spectrometry it is natural to assume that all components of the vector $\theta$ are nonnegative. Since $\mu(x, \theta)$ is a linear function of the estimated parameters, the maximum likelihood estimator is found as the solution to the system of equations

$$
\frac{\partial \ell(y, \theta)}{\partial \theta_{\alpha}}=-\sum_{i=1}^{n} \psi_{\alpha}\left(x_{i}\right)+\sum_{i=1}^{n} \frac{y_{i} \psi_{\alpha}\left(x_{i}\right)}{\boldsymbol{\theta}^{T} \psi\left(x_{i}\right)}, \alpha=1, \ldots, m
$$

or

$$
\sum_{i=1}^{n} w_{i} y_{i} \psi_{\alpha}\left(x_{i}\right)=\sum_{i=1}^{n} \psi_{\alpha}\left(x_{i}\right)
$$

where

$$
w_{i}^{-1}=\theta^{T} \Psi\left(x_{i}\right)
$$

After some simplification, this same system can be written in the following matrix notation:

$$
\Psi^{T} W \Psi \theta=\Psi^{T} W y
$$


where $W$ is an $n \times n$ diagonal matrix with the $i$-th diagonal entry $w_{i}$; the $n \times(m+1)$ matrix $\Psi$ has the $(i, \alpha)^{t h}$ entry given by $\psi_{\alpha}\left(x_{i}\right) ; \theta$ is the $(m+1) \times 1$ vector with $\alpha^{\text {th }}$ entry $\theta_{\alpha}$; and $\mathrm{y}$ is the $n \times 1$ vector with $i$-th entry $y_{i}$ for $i=1,2, \ldots, n$ and $\alpha=0,1, \ldots, m$. Let $M(\theta)$ equal $\Psi \mathrm{W} \Psi$ and $Y(\theta)$ equal $\Psi^{\prime} W \mathrm{y}$, then the solution for $\theta$ is given by

$$
\hat{\theta}=M^{-1}(\theta) Y(\theta)
$$

The above equation for the solution of $\theta$ is a typical object for the "fixed point" method in numerical mathematics or for the "iterative reweighted least squares" estimation method in statistics; compare with $[14,15]$. At the $s$-th iteration, the new estimate of $\boldsymbol{\theta}$ is obtained from

$$
\hat{\boldsymbol{\theta}}_{s+1}=M^{-1}\left(\hat{\boldsymbol{\theta}}_{s}\right) Y\left(\hat{\boldsymbol{\theta}}_{s}\right)
$$

Under some reasonably achievable regularity conditions, it can be shown that

$$
\lim _{s \rightarrow \infty} \hat{\boldsymbol{\theta}}_{s}=\hat{\boldsymbol{\theta}}
$$

where $\hat{\theta}$ is the maximum likelihood estimator.

When the spectra of all elements in the mixture are well disjoint, i.e. their peaks do not overlap, then the following assumption looks very reasonable. There are disjoint sets $I_{\alpha}$, such that

$$
\psi_{\alpha}\left(x_{i}\right)=\left\{\begin{array}{rr}
\phi_{\alpha}\left(x_{i}\right) \geq 0, \quad i \in I_{\alpha} \\
0, \quad \text { otherwise }
\end{array}\right.
$$

where $I_{\alpha} \cap I_{\beta}=0$ when $\alpha \neq \beta$. Under this set of assumptions, $M(\theta)$ is a diagonal matrix with diagonal elements given by

$$
M_{\alpha \alpha}(\theta)=\theta_{\alpha}^{-1} \sum_{i \in I_{\alpha}} \phi_{\alpha}\left(x_{i}\right)
$$

for $\alpha=1,2, \ldots, m$. Similarly, for this case, the elements in the vector $Y(\theta)$ are given by

$$
Y_{\alpha}(\theta)=\theta_{\alpha}^{-1} \sum_{i \in I_{\alpha}} y_{i}
$$


again for $\alpha=1,2, \ldots, m$. From equations (12) and (13), we have

$$
\hat{\theta}=\frac{\sum_{i \in I_{\alpha}} y_{i}}{\sum_{i \in I_{\alpha}} \phi_{\alpha}\left(x_{i}\right)}
$$

and

$$
\operatorname{Var}\left(\hat{\theta}_{\alpha}\right)=\frac{\sum_{i \in I_{\alpha}} \operatorname{Var}\left(y_{i}\right)}{\left(\sum_{i \in I_{\alpha}} \phi_{\alpha}\left(x_{i}\right)\right)^{2}}=\frac{\theta_{\alpha}}{\sum_{i \in I_{\alpha}} \phi_{\alpha}\left(x_{i}\right)}
$$

for $\alpha=1,2, \ldots, m$. An estimate of $\operatorname{Var}\left(\hat{\theta}_{\alpha}\right)$ can be found by substituting $\hat{\theta}_{\alpha}$ for $\theta_{\alpha}$. Being a sum of random variables, which have Poisson distributions, $\hat{\theta}_{\alpha}$ also has the Poisson distribution. Thus, we conclude that the method which is standardly used to estimate activities (see Section 3) may be considered as an approximate one and there exist statistically more efficient methods. The iterative procedure described above is easily realized with modern software. The superiority becomes especially evident when the background noise is comparable with the height peaks and peaks from various elements are overlapping. 


\section{Appendix 3. Models and Analysis of Sampling Experiments}

Models. In the sampling experiment there are at least three types of uncertainties, which have to be considered and modeled:

1. Sampling of items from the population of items of different types, exposure, cleaning treatment, etc.

2. All emitting processes are random. Correspondingly, all the observed "counts" must be treated as random variables.

3. Observational errors, for instance, the presence of a background noise, can be a significant addition to the above mentioned sources of uncertainty.

The uncertainties of type (a) are probably the largest one. A proper inventory and stratification procedure may reduce them, but not remove them completely.

One of the simplest models used in many applied studies may be described in the "waste characterization" setting as follows:

Let $x_{0}$ be a contamination level (e.g. TRU activity) of an item at some stage of its active life. Then the probability that this level will be increased by $x$ does not depend upon the existing contamination level $x_{0}$. In other words, there are equal chances to increase contamination by a given amount either for a "dirty" or "clean" item. In the language of probability, our assumption can be described as

$$
\text { Probability }\left(x_{0} \leq X<x_{0}+x\right)=\text { Probability }(0 \leq X<x)
$$

The corresponding probability density function (pdf) that satisfies equation (1) is well known:

$$
p(x)=\sigma^{-1} e^{-x / \sigma},
$$

and is called the exponential distribution; see [15] for more details. For the pdf (2), the expected value of contamination equals $\sigma$. We understand that model (1) and (2) are an approximate description of reality. Nevertheless, we prefer to use this model versus the more traditional Gaussian or normal distribution, because unlike the latter it has 
a better physical explanation in our setting. In addition, it results in relatively simple estimation procedures.

The probability models for the uncertainties of type (b) are well known in nuclear physics and we confine our choice to the Poisson distribution where the probability that we observe $x$ counts in a given unit of time is

$$
p(x)=\frac{\lambda^{x} e^{-\lambda}}{x !}
$$

In this particular setting, the observational errors are comparatively small and the choice of the models can vary for different measurement procedures. In this appendix we address the first two types of uncertainties.

Analysis of proportion. Let us assume that in a sampling experiment we can measure two components $x_{1}$ and $x_{2}$. In our instance, $\alpha$-activity of TRU may be selected as $x_{1}$, and $\alpha$-activity of all other isotopes as $x_{2}$. Let us further assume that the ratio of $x_{1}$ and $x_{2}$ is rather stable. In terms of model (2) it means that

$$
\sigma_{2}=k \sigma_{1}
$$

where $\sigma_{\alpha}, \alpha=1,2$, is the expected value of the corresponding distribution.

Assuming that the realizations $x_{1 i}$ and $x_{2 i}, i=1, \ldots, n$, are independent and known exactly, we can introduce the following maximum likelihood function

$$
L\left(x_{1}, x_{2}, \sigma, k\right)=\prod_{i=1}^{n} \sigma^{-2} k^{-1} \exp \left(\sigma^{-1}\left(x_{1 i}+k^{-1} x_{2 i}\right)\right)
$$

where $\sigma=\sigma_{1}$.

Straightforward maximization of (5) leads to the maximum likelihood estimator:

$$
\hat{k}=\frac{\bar{x}_{2}}{\bar{x}_{1}}
$$

where $\bar{x}_{\alpha}=\frac{1}{n} \sum_{i=1}^{n} x_{\alpha i}, \alpha=1,2$.

When $x_{\alpha}$ are activities of some elements, then they may be measured through some 
counting procedures, i.e. instead of $x_{\alpha i}$ we know $y_{\alpha i}$, which are distributed (see (3)) as

$$
p\left(y_{\alpha} \mid x_{\alpha i}\right)=\frac{x_{\alpha i}^{y_{\alpha}} e^{-x_{\alpha i}}}{y_{\alpha} !}
$$

and

$$
\begin{aligned}
p\left(y_{\alpha} \mid \sigma_{\alpha}\right) & =\int_{0}^{\infty} p\left(y_{\alpha} \mid x_{\alpha i}\right) p\left(x_{\alpha i} \mid \sigma_{\alpha}\right) d x_{\alpha i} \\
& =\int_{0}^{\infty} \frac{x_{\alpha i}^{y_{\alpha}} e^{-(1+1 / \sigma \alpha) x_{\alpha i}}}{y_{\alpha} ! \sigma_{\alpha}} d x_{\alpha i} \\
& =\frac{\sigma_{\alpha}^{y_{\alpha}}}{\left(1+\sigma_{\alpha}\right)^{y_{\alpha}+1}} .
\end{aligned}
$$

In (8) we used the formula

$$
\int_{0}^{\infty} x^{y-1} e^{-a x} d x=a^{-y} \Gamma(y)
$$

Since $\sigma_{2}=k \sigma_{1}$ and from (8) we have that

$$
L\left(\mathrm{y}_{1}, \mathrm{y}_{2}, \sigma, k\right)=\prod_{i=1}^{n} \frac{\sigma^{y_{1 i}}(k \sigma)^{y_{2 i}}}{(1+\sigma)^{y_{1 i}+1}(1+k \sigma)^{y_{2 i}+1}}
$$

where $\mathrm{y}_{\alpha}$ is the $n \times 1$ vector of $y_{\alpha i}, \alpha=1,2$, and $\sigma=\sigma_{1}$.

Again, straightforward maximization of (9) shows that the maximum likelihood estimator for $k$ is

$$
\hat{k}=\frac{\bar{y}_{2}}{\bar{y}_{1}}
$$

where $\bar{y}=\frac{1}{n} \sum_{i=1}^{n} y_{\alpha i}$ and $\alpha=1,2$.

Alternative model. In the previous section, we assumed that $x_{1 i}$ and $x_{2 i}$ are "generated" independently of each other. The link between $\sigma_{1}$ and $\sigma_{2}$ is actually some loose link between the means of these two random variables. Perhaps in the case of contaminated objects, it is expedient to assume a stronger relationship between $x_{1 i}$ and $x_{2 i}$. Namely, we assume that

$$
x_{2 i}=k x_{1 i}
$$


In other words, the ratio, $x_{2 i} / x_{1 i}$, between various components for all selected items is the same. Do not forget that we observe "counts" $y_{1 i}$ and $y_{2 i}$, in general. Unlike the previous section, where

$$
p\left(y_{1}, y_{2}, \mid \sigma\right)=p\left(y_{1} \mid \sigma\right) p\left(y_{2} \mid \sigma\right)
$$

i.e. $y_{1}$ and $y_{2}$ are independent, now observations are dependent and we cannot use (8) to derive the common probability density for $y_{1}$, and $y_{2}$.

However, it still has a simple form, which may be found through direct integration:

$$
\begin{aligned}
p\left(y_{1}, y_{2} \mid \sigma\right) & =\int_{0}^{\infty} p\left(y_{1}, y_{2} \mid x_{1}\right) p\left(x_{1} \mid \sigma\right) d x_{1}, \\
& =\int_{0}^{\infty} \frac{x_{1}^{y_{1}} e^{-x_{1}}}{y_{1} !} \frac{\left(k x_{1}\right)^{y_{2}} e^{-k x_{1}}}{y_{2} !} \frac{1}{\sigma} e^{-x_{1} / \sigma} d x_{1}, \\
& =\frac{\left(y_{1}+y_{2}\right) !}{y_{1} ! y_{2} !} \frac{(k \sigma)^{y_{2}} \sigma^{y_{1}}}{(k \sigma+\sigma+1)^{\left(y_{1}+y_{2}+1\right)}}
\end{aligned}
$$

Now the maximum likelihood function equals

$$
L\left(\mathbf{y}_{1}, \mathbf{y}_{2}, \sigma, k\right)=\prod_{i=1}^{n} \frac{\left(y_{1 i}+y_{2 i}\right) !}{y_{1 i} ! y_{2 i} !} \cdot \frac{\sigma^{y_{1 i}}(k \sigma)^{y_{2 i}}}{(k \sigma+\sigma+1)^{y_{1 i}+y_{2 i}+1}}
$$

Amazingly, after direct maximization of (14), we have the following maximum likelihood estimator for $k$ :

$$
\hat{k}=\frac{\bar{y}_{1}}{\bar{y}_{2}}
$$

Thus, in all three cases considered, we have the same formula for $\hat{k}$. In all three cases we need to know the arithmetic means $\left(\bar{y}_{1}\right.$ and $\left.\bar{y}_{2}\right)$ to get the maximum likelihood estimators. For the reader with some statistical background, we note that $\bar{y}_{1}$ and $\bar{y}_{2}$ are sufficient statistics for $k$ and $\sigma$. At a less technical level, one can say that $\bar{y}_{1}$ and $\bar{y}_{2}$ contain all the information in the frame of the considered models about $k$ and $\sigma$.

Nuisance parameters approach. In the previous sections, it was assumed that the contamination of items has the exponential distribution. In spite of the existence of very reasonable physical background to use this distribution, the validity of the assumptions, which were made, can be questioned. In this section we present another approach, which is free from those assumptions (see (1), (2), (4)). However, the assumptions (3) 
and (11) are essential for the reported result. These two assumptions state that the distribution of activity (counts) measurements have the Poisson distribution and that the ratio of activities is constant for all items. Our main objective is to find this ratio.

From (3) and (11) we can derive that

$$
L\left(\mathrm{y}_{1}, \mathrm{y}_{2}, x_{1}, k\right)=\prod_{i=1}^{n} \frac{x_{1 i}^{y_{1 i}} e^{-x_{1 i}}}{y_{1 i} !} \frac{\left(k x_{1 i}\right)^{y_{2 i}} e^{-k x_{1 i}}}{y_{2 i} !}
$$

Variables $x_{1 i}, i=1, \ldots, n$, may be considered as nuisance parameters. Direct maximization of (16) shows immediately that we can construct the maximum likelihood estimator for $k$, which does not depend upon $x_{1 i}$, and is given by

$$
\hat{k}=\frac{\bar{y}_{1}}{\bar{y}_{2}}
$$

Again, we have come to the same formula. 


\section{PLOTS}

\section{FIGURES 1 - 10}

Legend Symbols Used in Figures

\begin{tabular}{|c|l|}
\hline$\times$ & $\begin{array}{l}\text { Observations where at least one component is below } \\
\text { detection level (Figures 1 and 2); }\end{array}$ \\
\hline$*$ & $\begin{array}{l}\text { Observations where all components are } \\
\text { known (Figures 1 and 2); }\end{array}$ \\
\hline $\mathbf{C , ~ G , ~ M , ~}$ \\
$\mathbf{P}$, and W & $\begin{array}{l}\text { Observations whose source is cell (C), } \\
\text { glass (G), metal (M), poly (P) or wipe (W) } \\
\text { (Figures 3 and 4); }\end{array}$ \\
\hline $\mathbf{U}, \mathbf{L}$, or 0 & $\begin{array}{l}\text { Observation derived using upper component activity } \\
\text { value (V), lower component value (L), } \\
\text { or derived when U = L (Figures 5 and 6); }\end{array}$ \\
\hline+ & $\begin{array}{l}\text { Observation (upper or lower bound) used in } \\
\text { regression analysis (Figures 7 - 10). }\end{array}$ \\
\hline & $\begin{array}{l}\text { Observations marked by their "sample ID" are } \\
\text { outlier observations or associated with the } \\
\text { observations which were discussed in text; } \\
\text { (see Table A5). }\end{array}$ \\
\hline
\end{tabular}


FIG. 1a LN TRU ACTIVITY VS LN GROSS ALPHA COMPLETE DATA SET

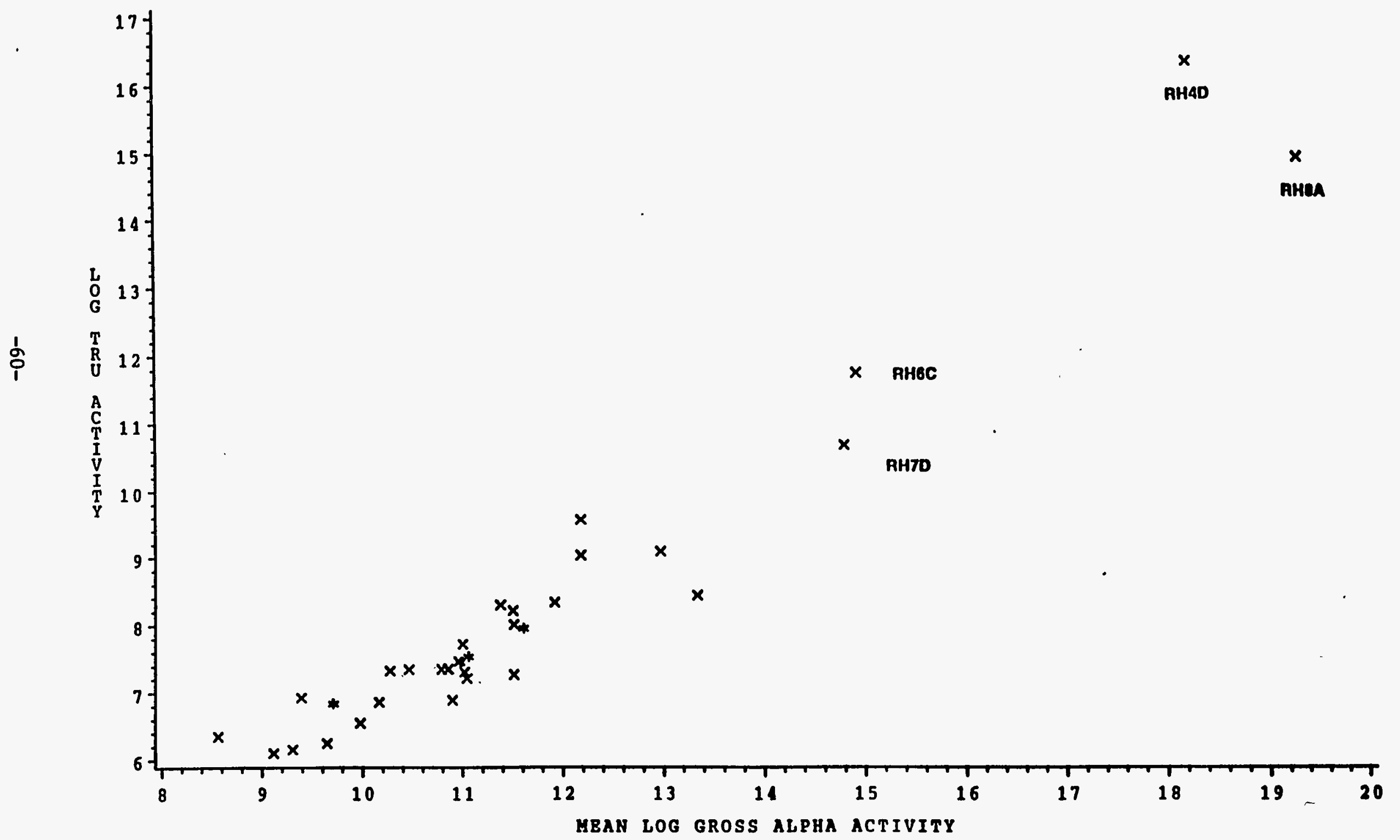


FIG. 1b LN CF252 ACTIVITY VS LN GROSS ALPHA COMPLETE DATA SET

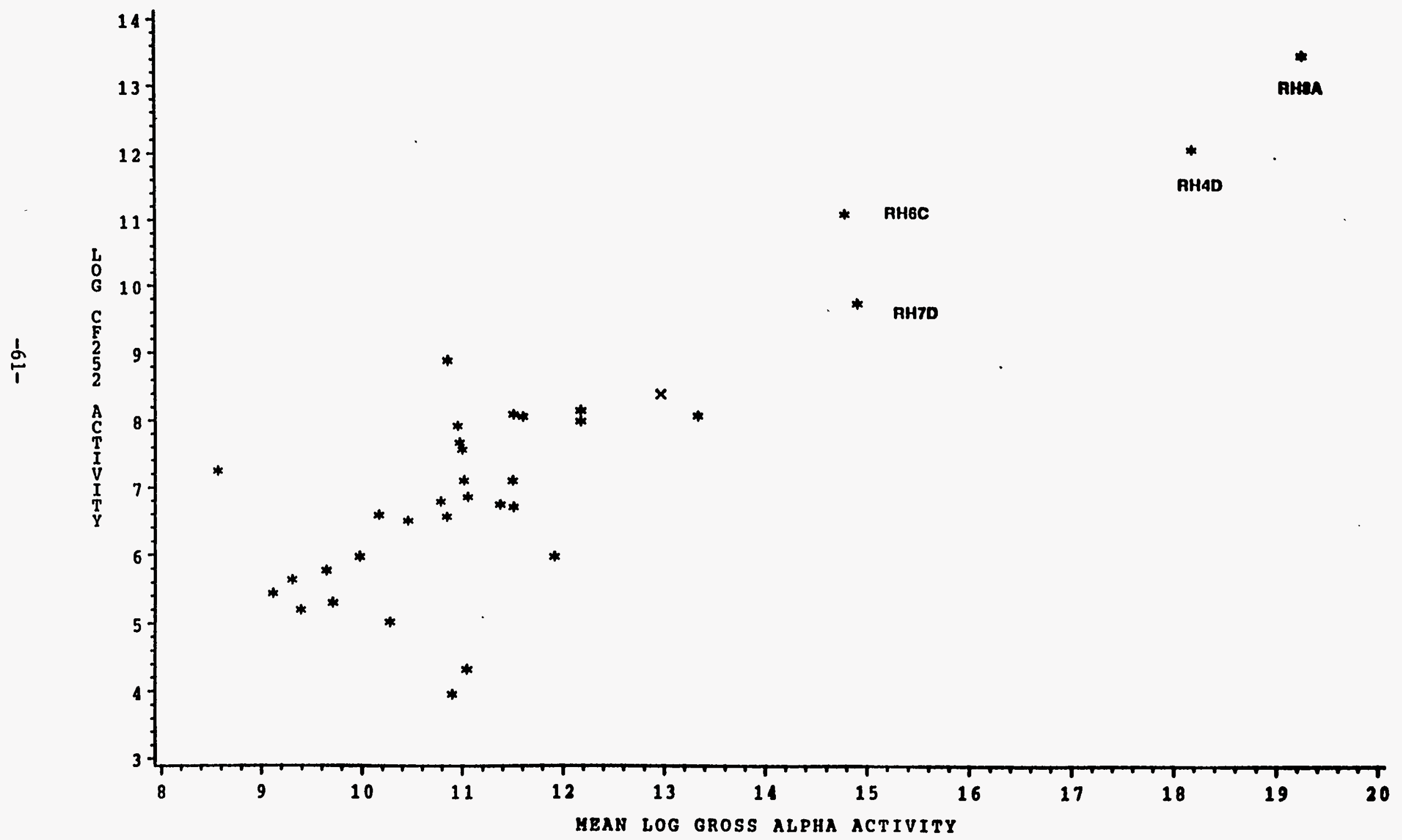




\section{FIG. 1c LN CM244 ACTIVITY VS LN GROSS ALPHA COMPLETE DATA SET}

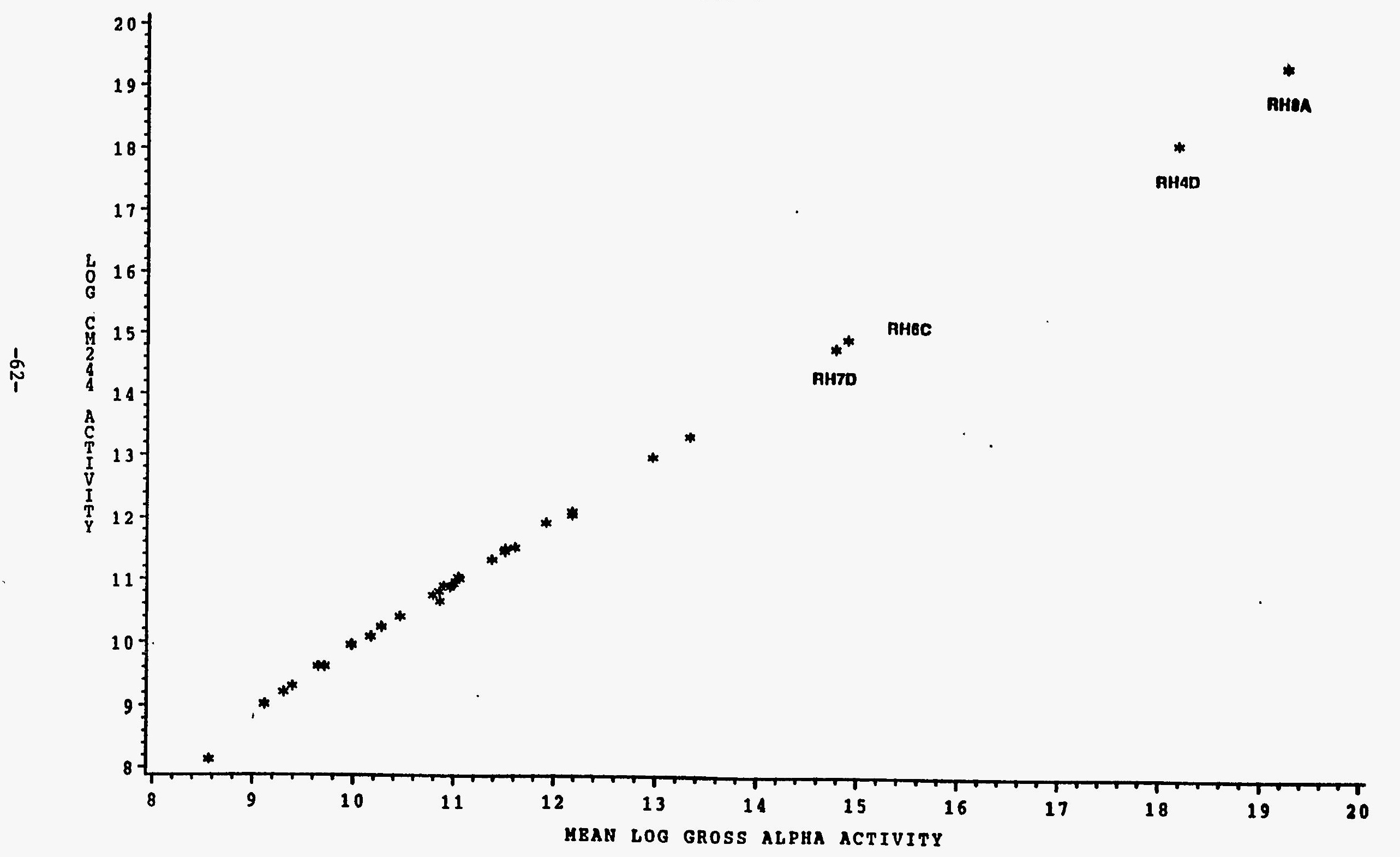



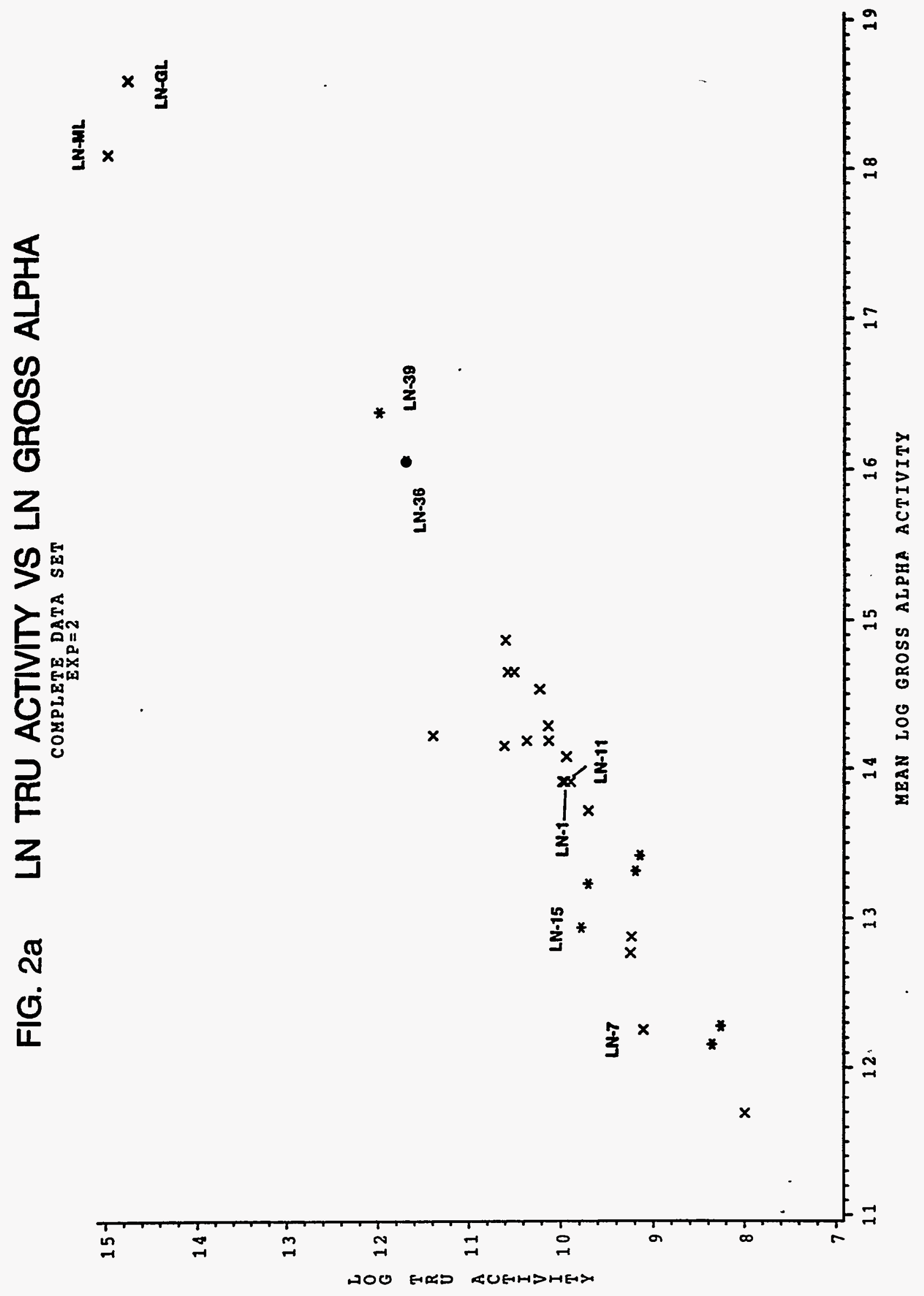

$\stackrel{\mathbb{N}}{\square}$
$\frac{1}{4}$

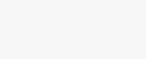




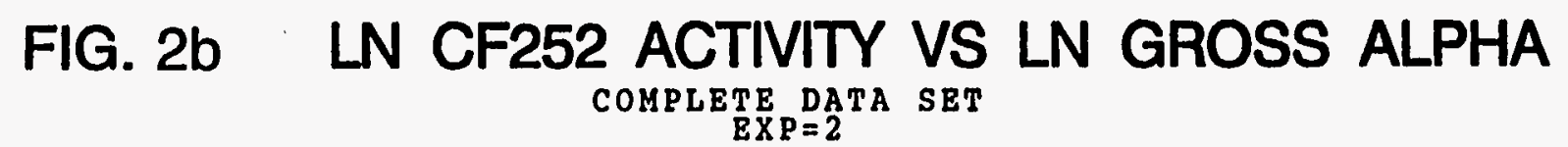

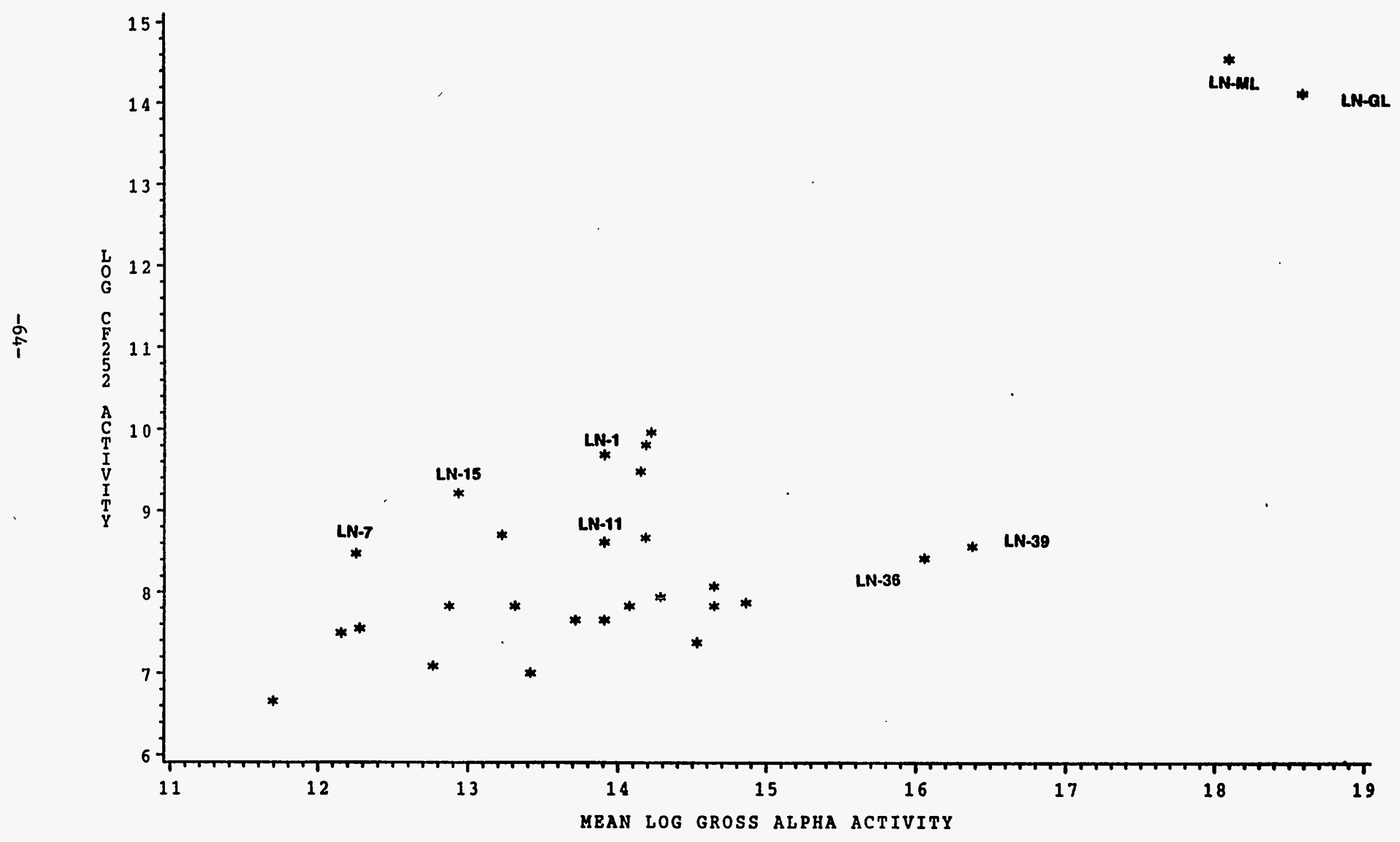


FIG. 2c LN CM244 ACTIVITY VS LN GROSS ALPHA COMPLETE DATA SBT

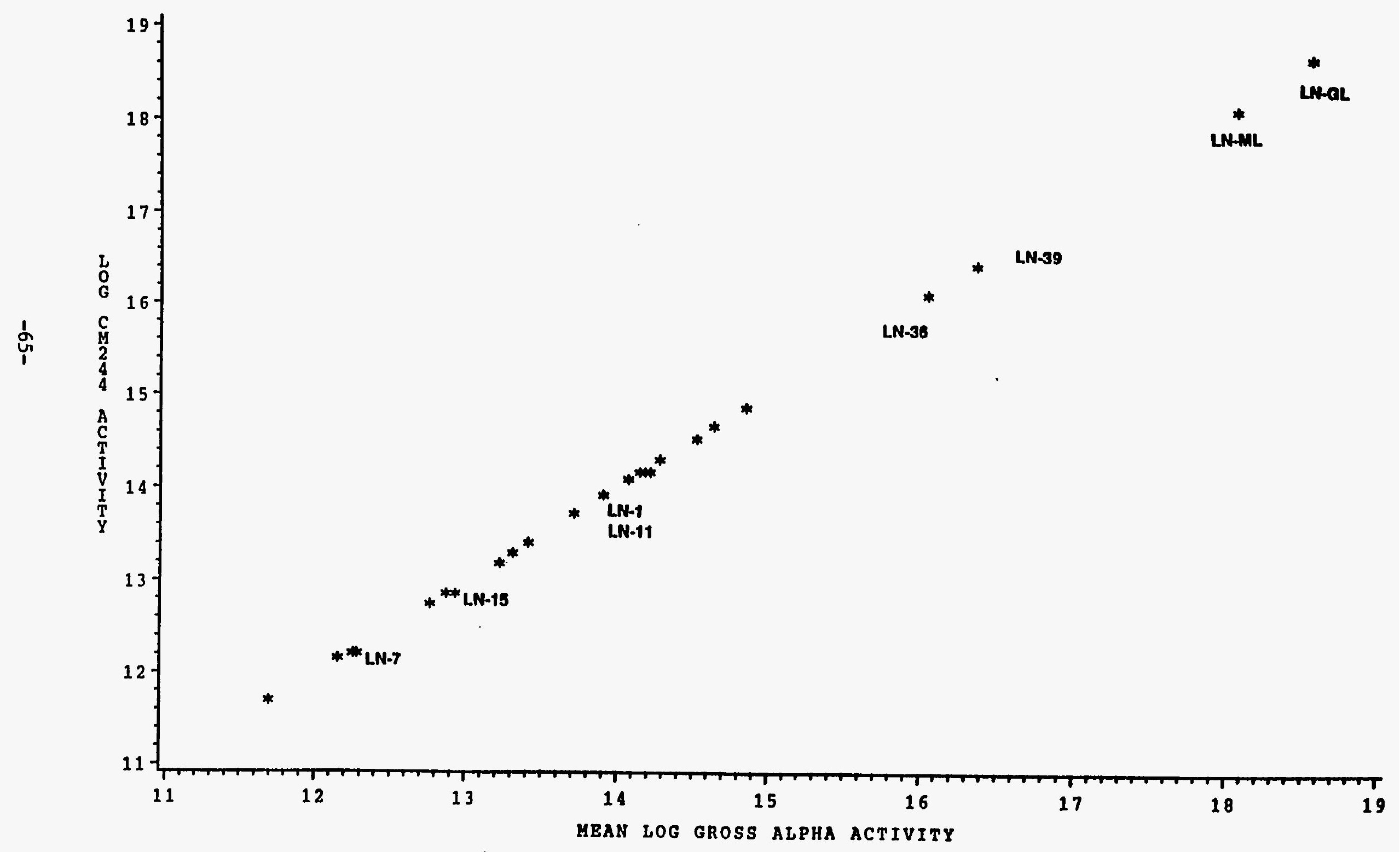




\section{FIG. 3a LN TRU ACTIVITY VS LN GROSS ALPHA COMPLETE DATA SET}

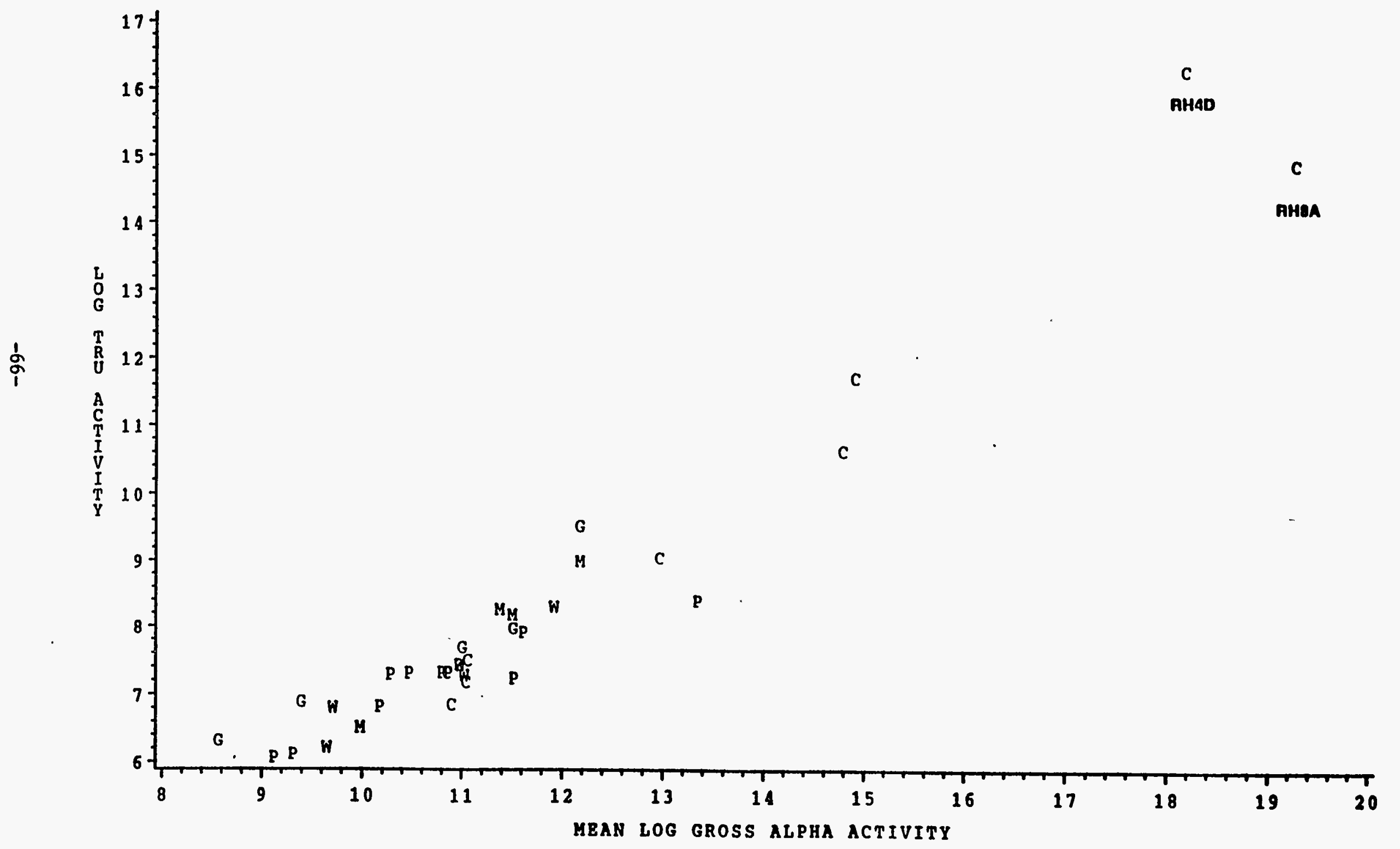


FIG. 3b LN CF252 ACTIVITY VS LN GROSS ALPHA COMPLETE DATA SET

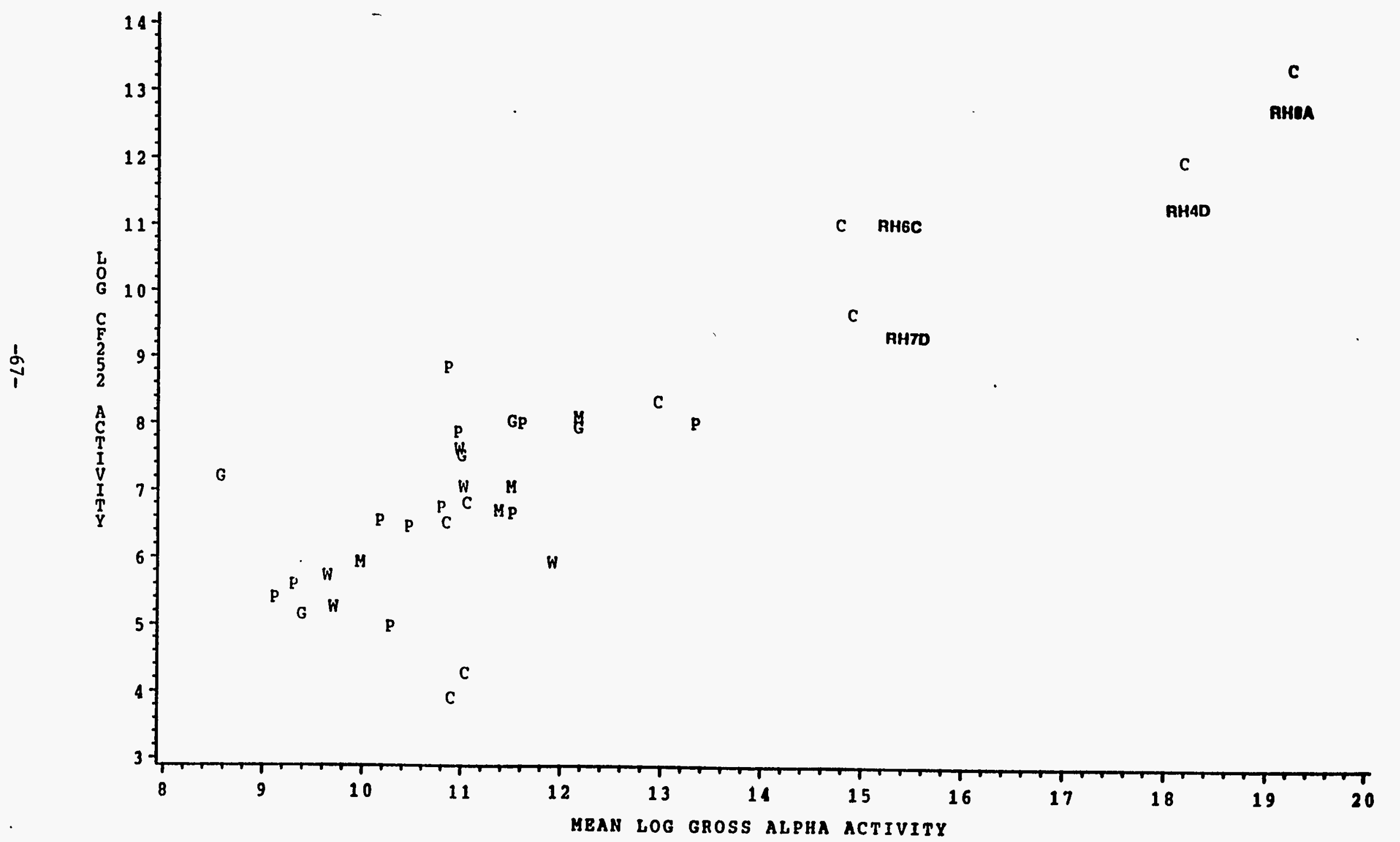


FIG. 3c LN CM244 ACTIVITY VS LN GROSS ALPHA COMPLETE DATA SBT

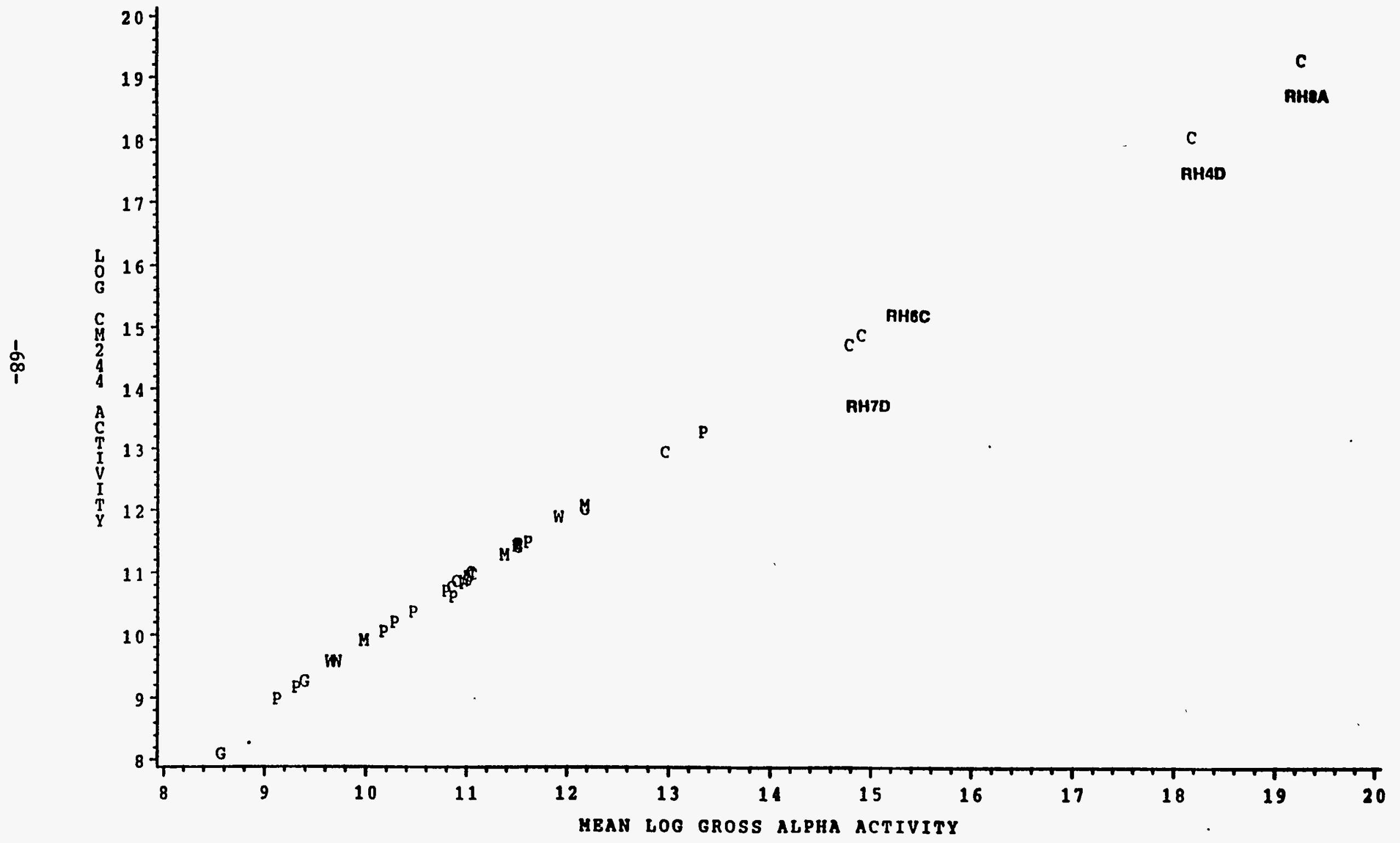


FIG. 4a LN TRU ACTIVITY VS LN GROSS ALPHA COMPLETE DATA SET
EXP=2

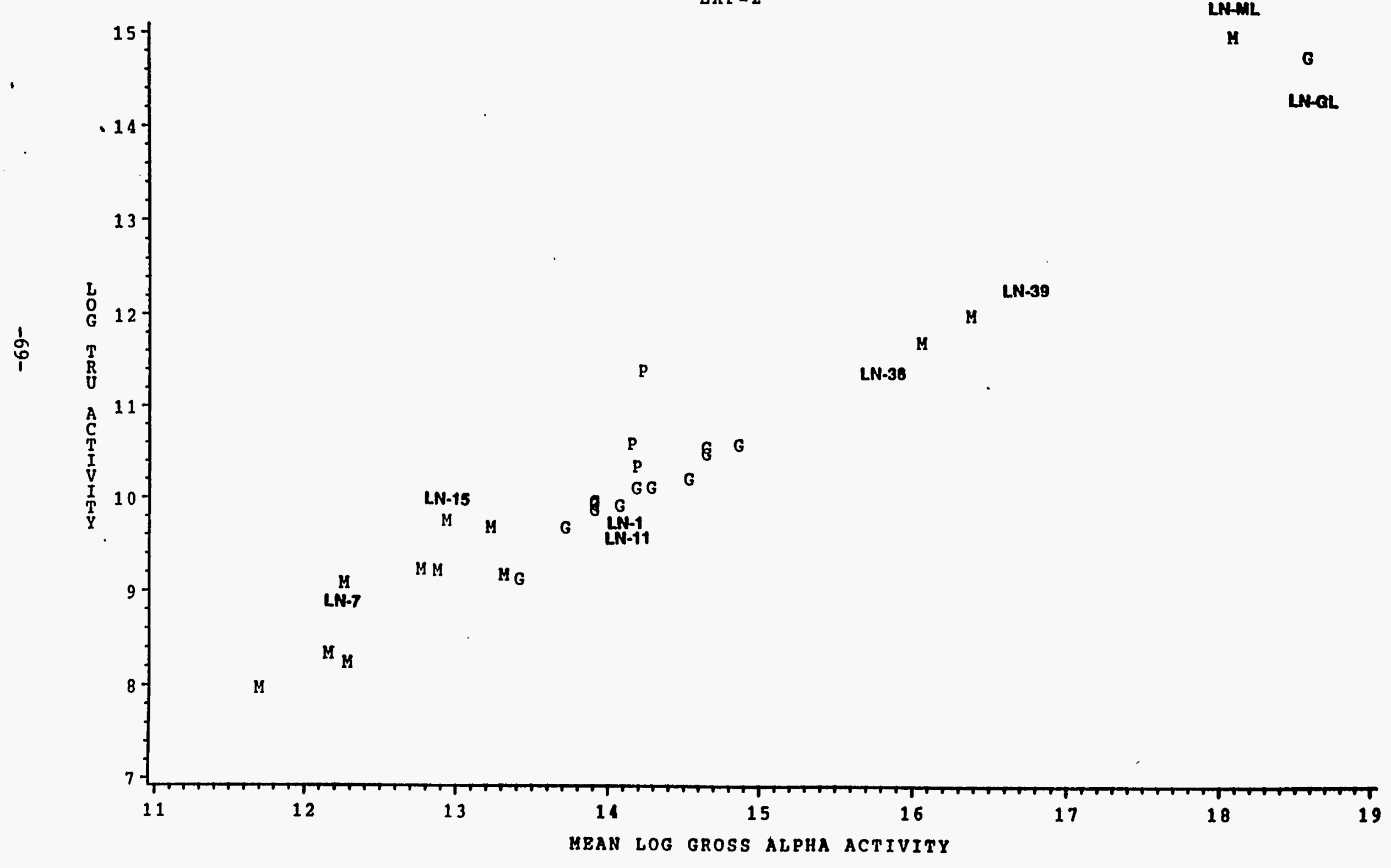


FIG. 4b LN CF252 ACTIVITY VS LN GROSS ALPHA COMPLETE DATA SET

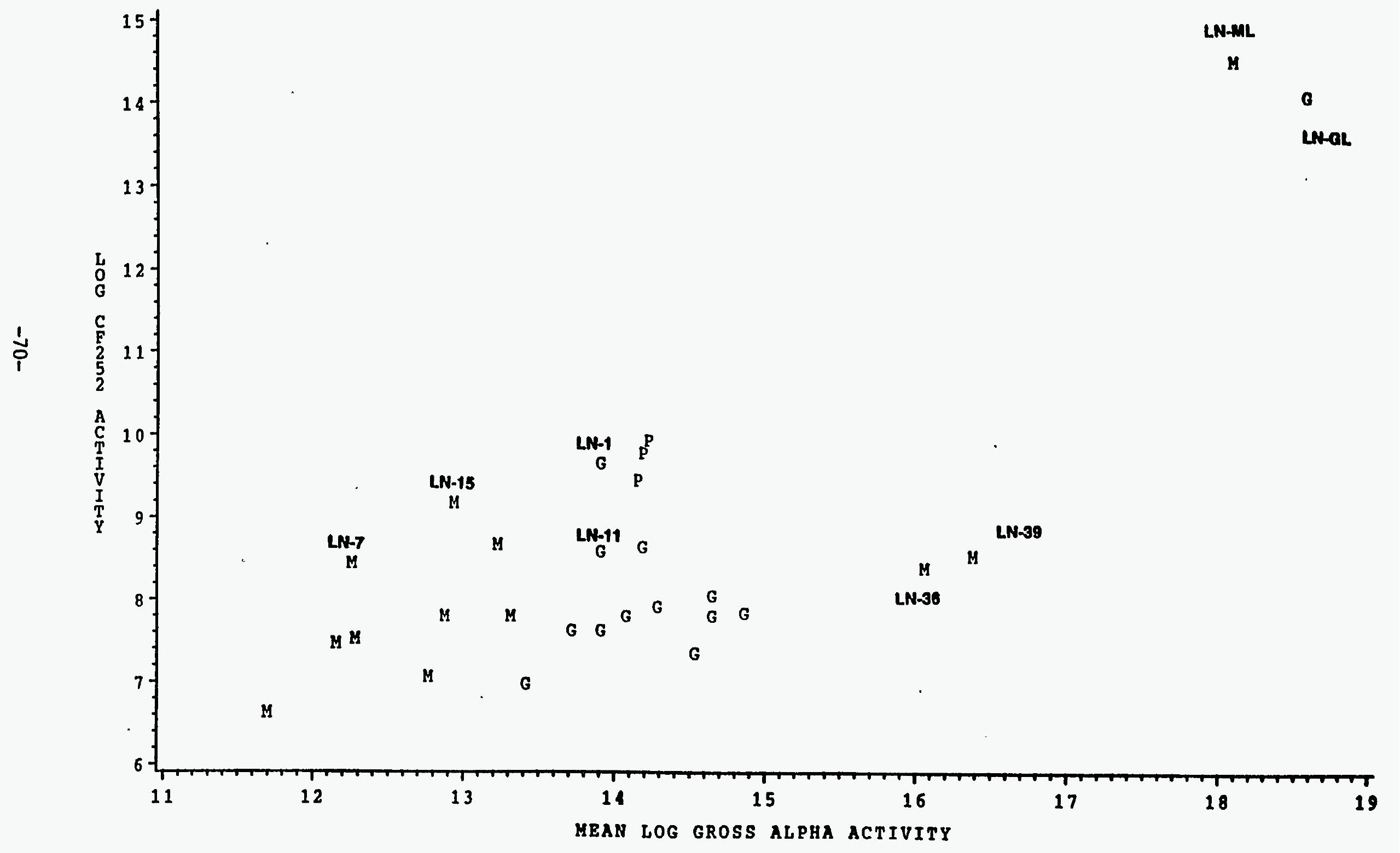



FIG. 4C . LN CM244 ACTIVITY $\underset{\text { COMPLETE DATA }}{\text { VST }}$ LN GROSS ALPHA

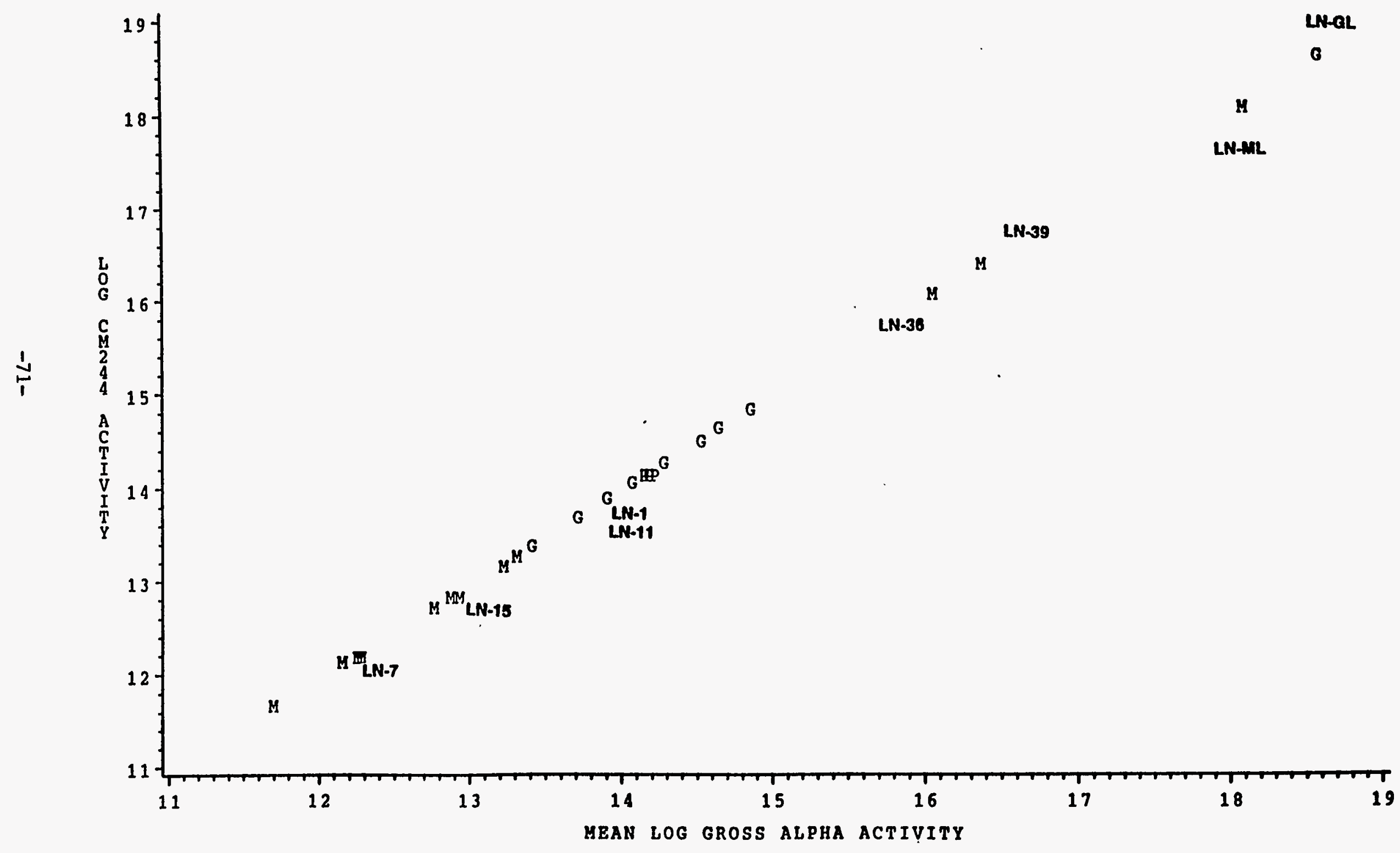


FIG. 5 . UPPER AND LOWER LN TRU ACTIVITIES VS MEAN LN GROSS ALPHA COMPLETE DATA SET

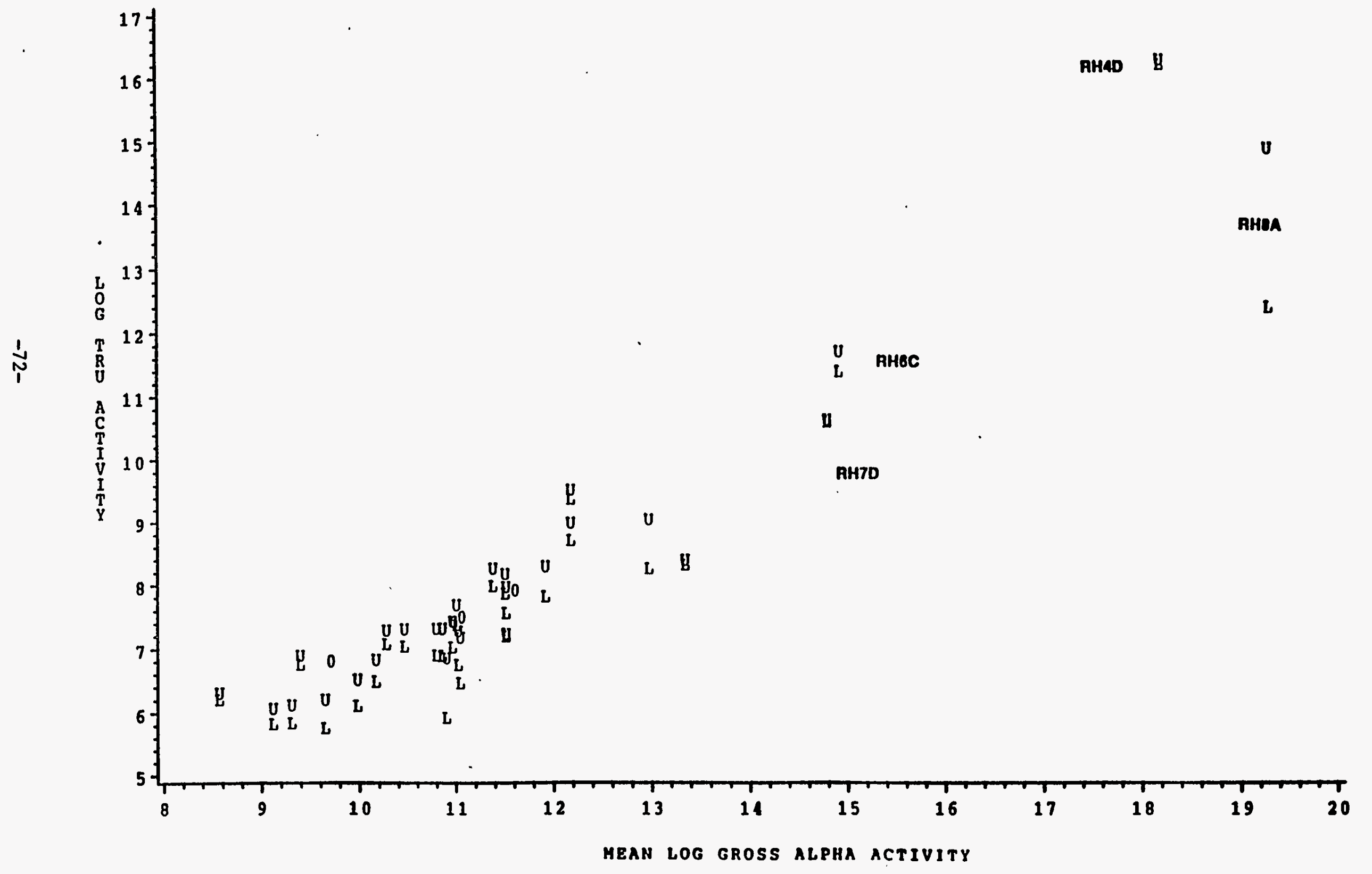




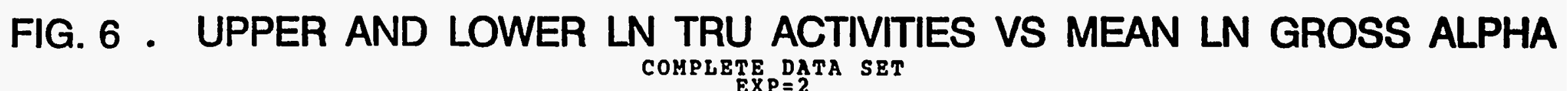

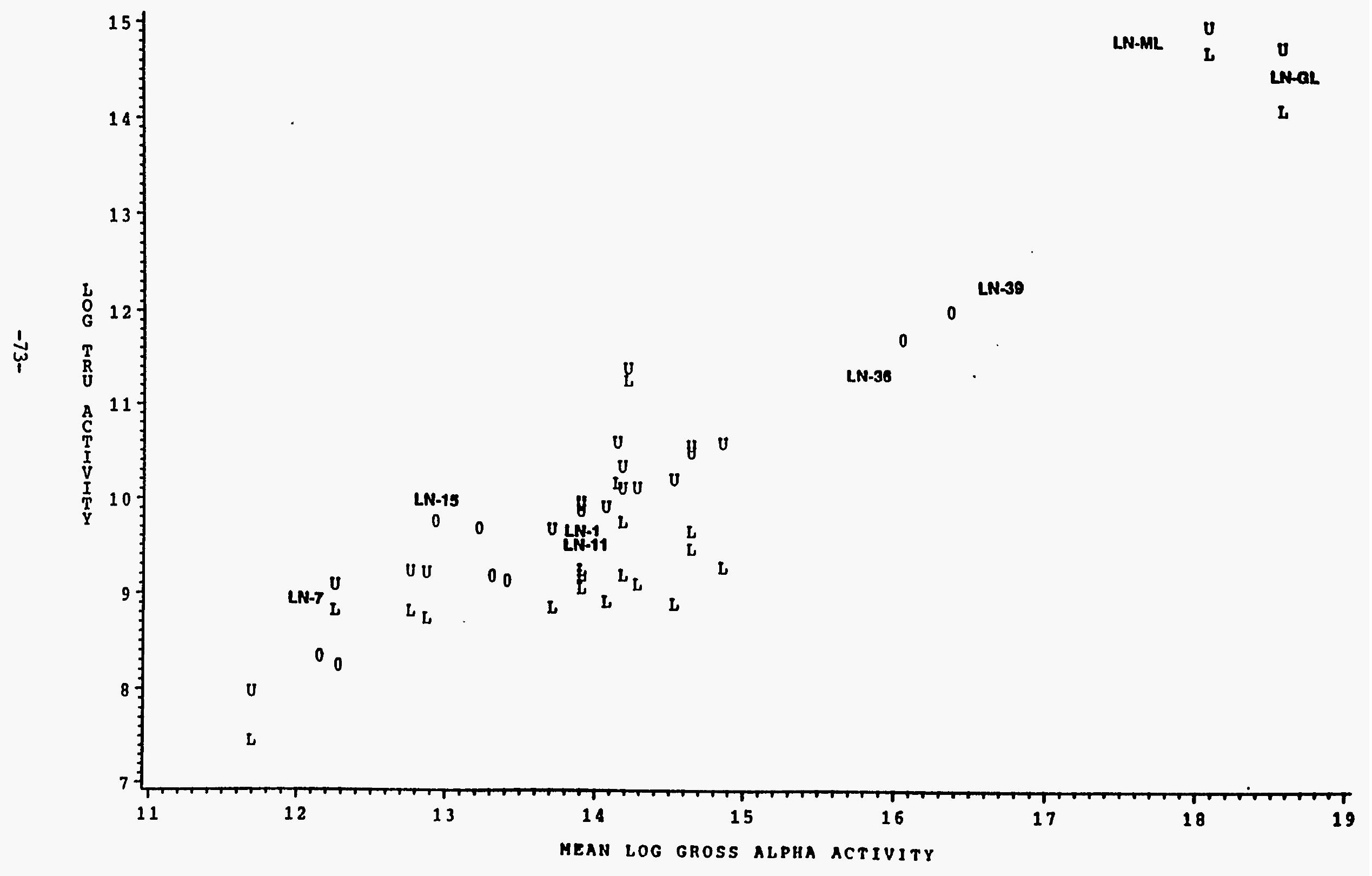


FIG. 7a. 1ST \& 2ND ORDER REGRESSION OF LN TRU (LOWER) ON LN GROSS ALPHA

EXPERIMENT 1--NO OBSERVATIONS OMITTED

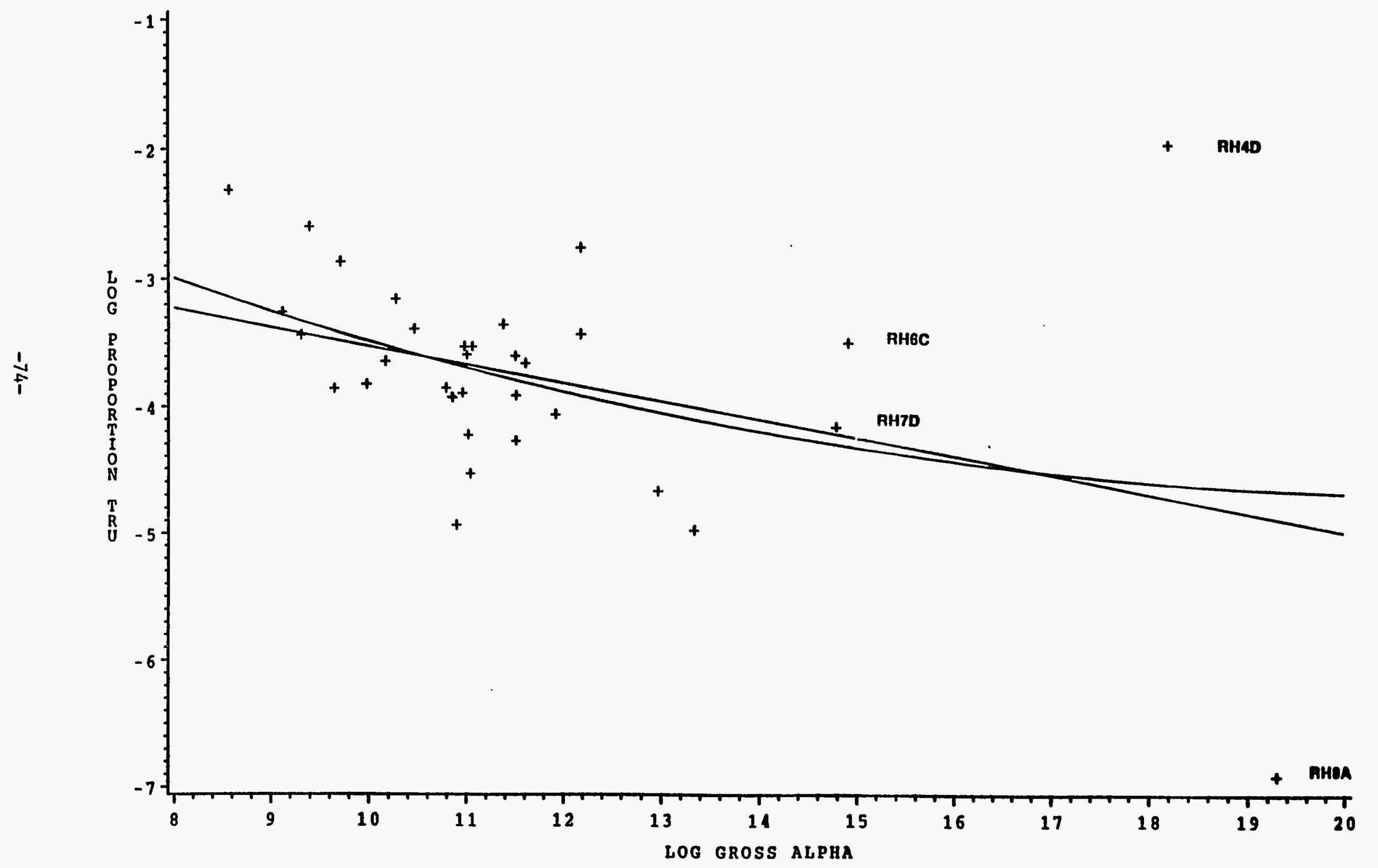


FIG. 7b. 1ST \& 2ND ORDER REGRESSION OF IN TRU (UPPER) ON LN GROSS ALPHA EXPERIHENT 1-NO OBSERVATIONS OMITTED

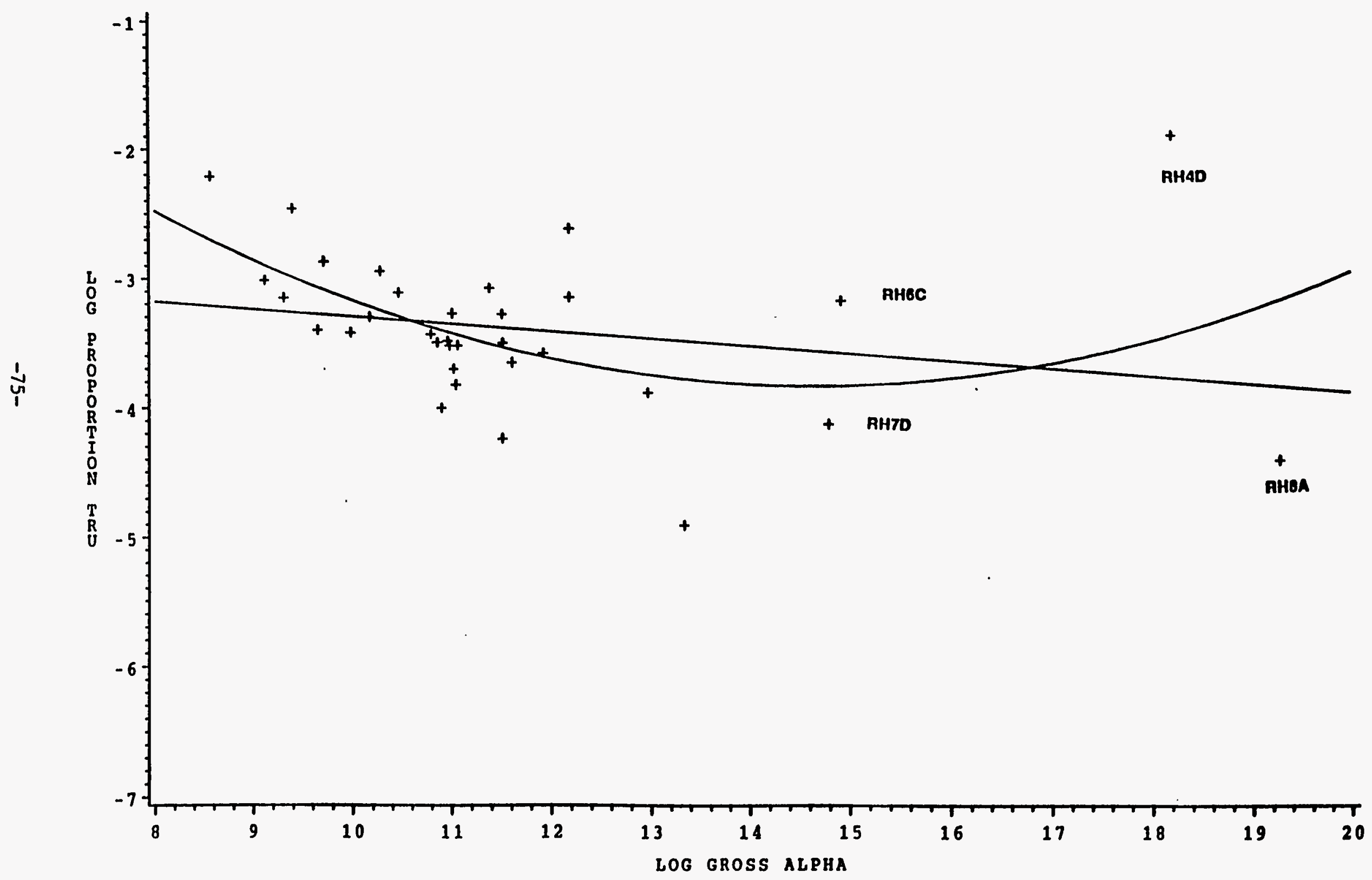


FIG. 7d. 1ST \& 2ND ORDER REGRESSION OF LN TRU (UPPER) ON LN GROSS ALPHA

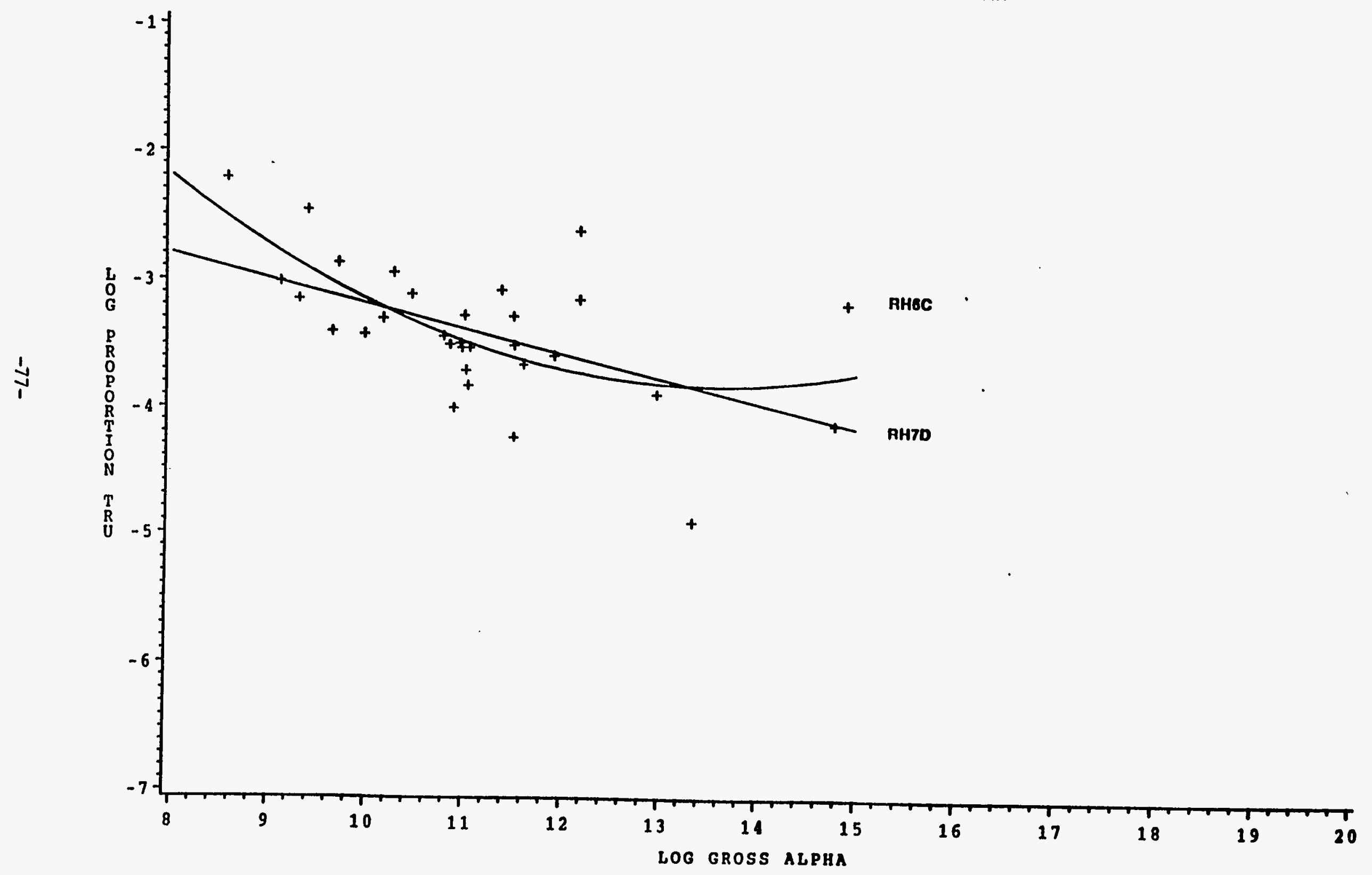


FIG. 8a. 1ST \& 2ND ORDER REGRESSION OF LN TRU (LOWER) ON LN GROSS ALPHA

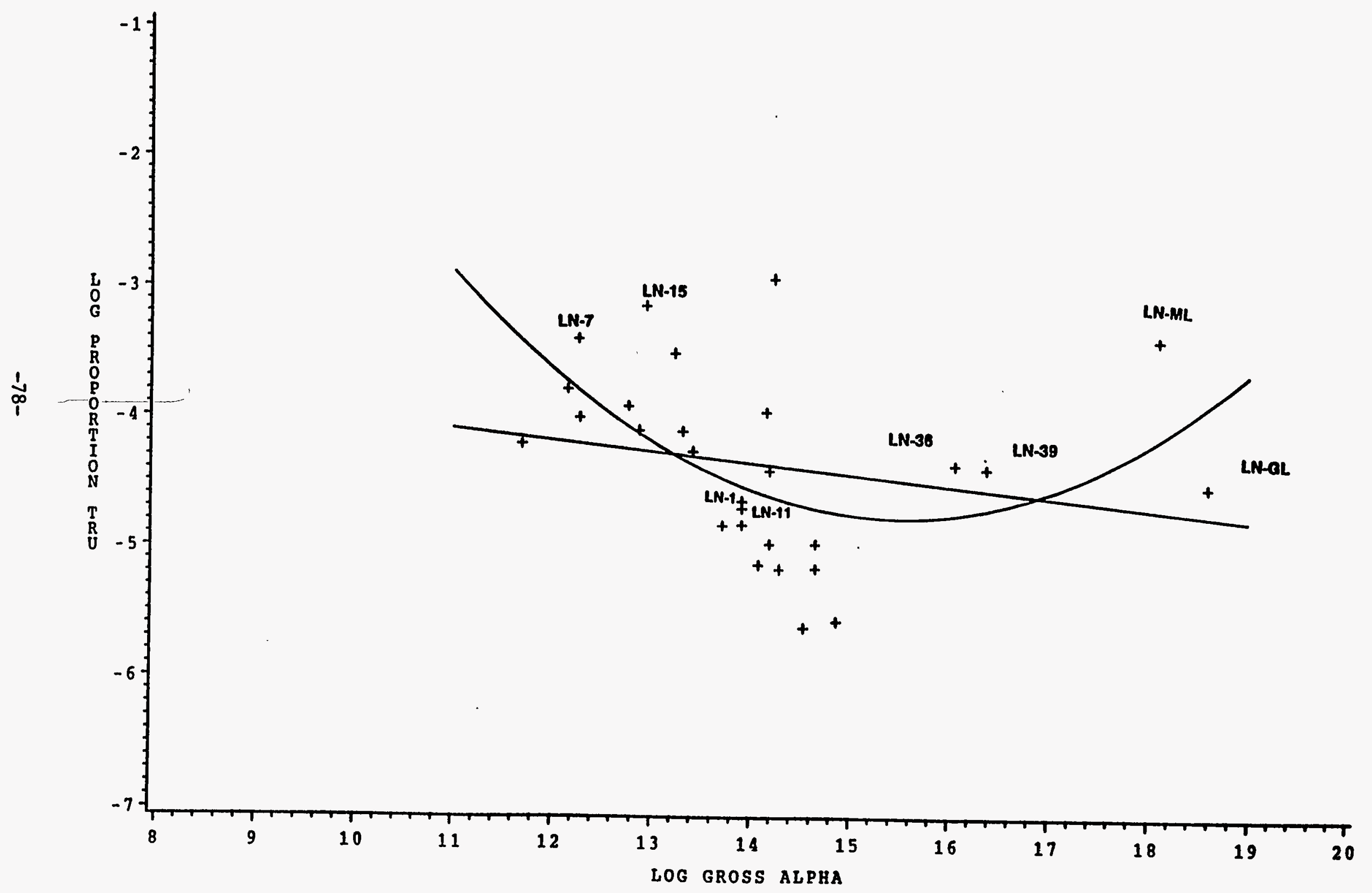


FIG. 8b. 1ST \& 2ND ORDER REGRESSION OF LN TRU (UPPER) ON LN GROSS ALPHA EXPERIMENT 2--NO OBSERVATIONS OMITTED

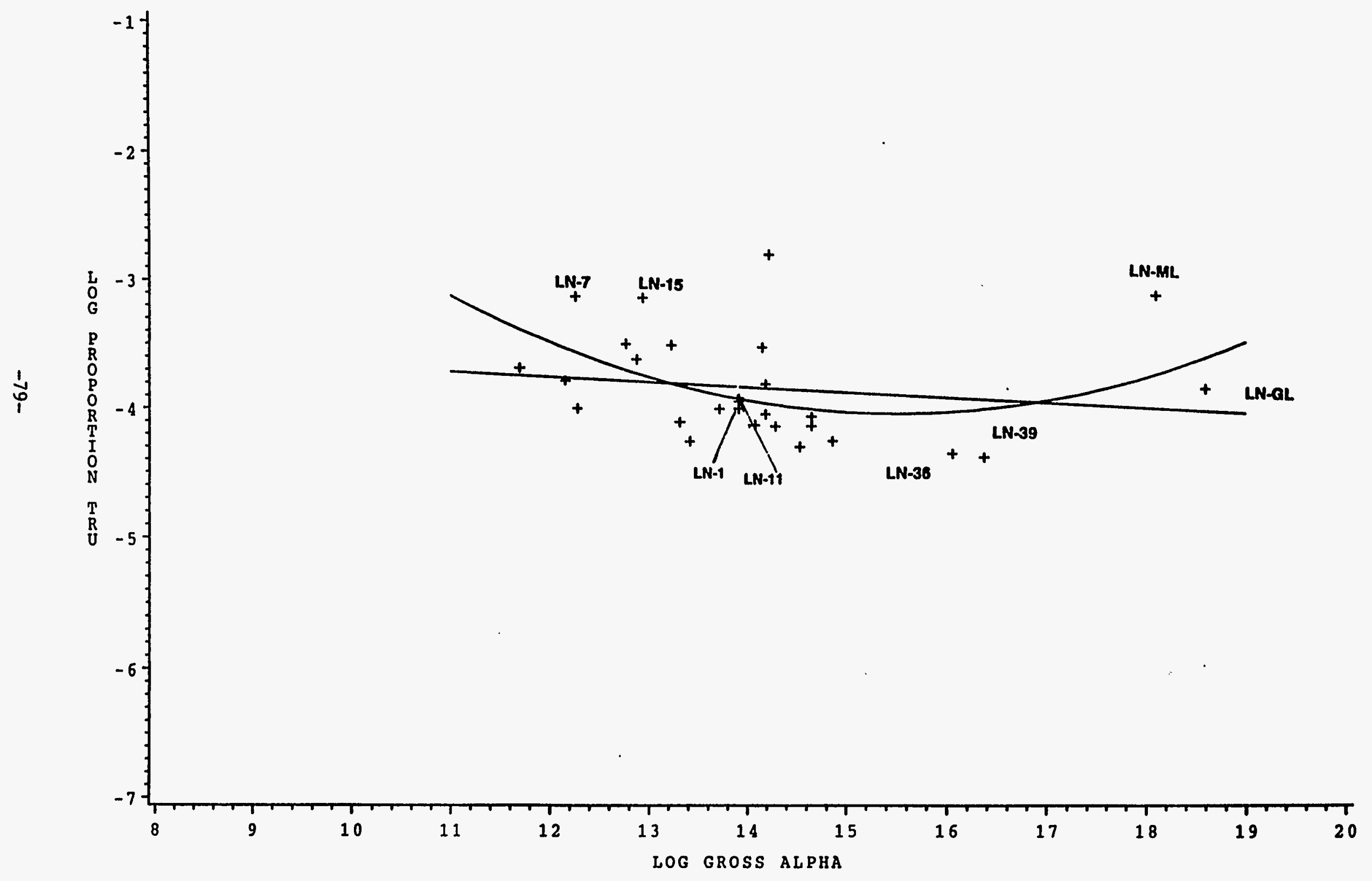


FIG. 8c. 1ST \& 2ND ORDER REGRESSION OF IN TRU (LOWER) ON LN GROSS ALPHA EXPERIMENT $2--2$ OUTLIER OBSERVATIONS OMITTED

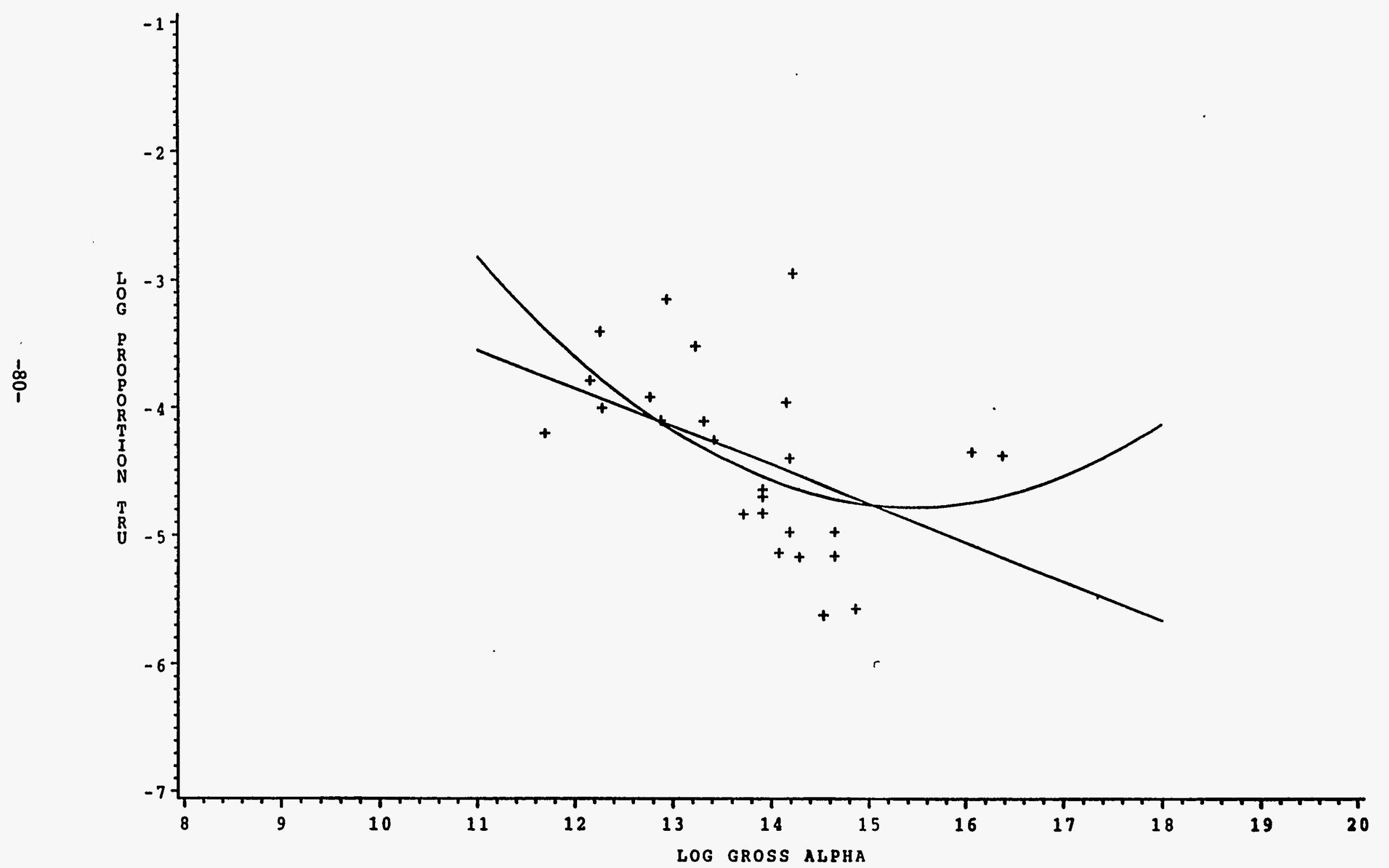


FIG. 8d. 1ST \& 2ND ORDER REGRESSION OF WN TRU (UPPEER) ON LN GROSS ALPHA

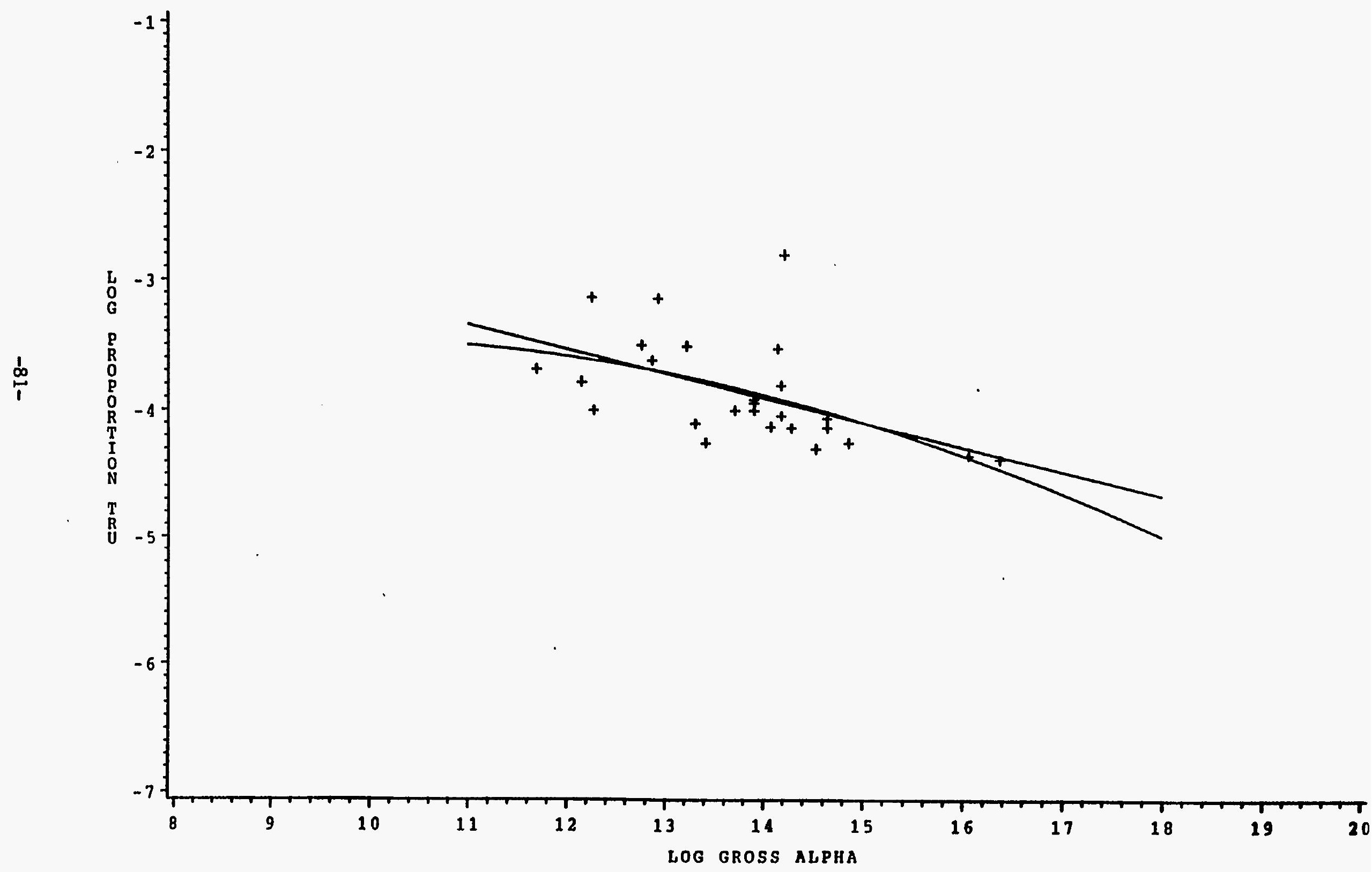



FIG. 9a. 1ST \& 2ND ORDER REGRESSION OF LN TRU (LOWER) ON LN GROSS ALPHA

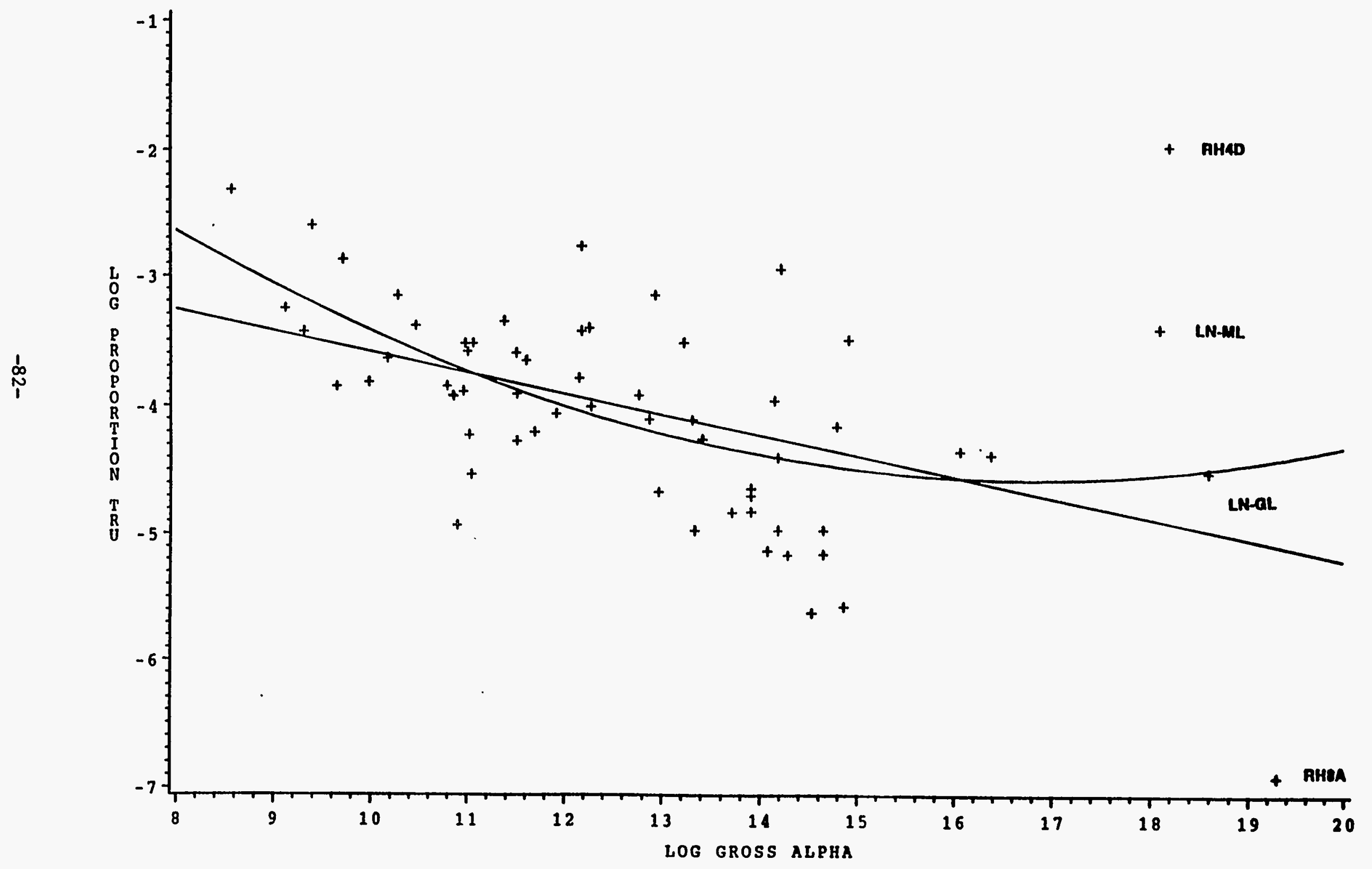


FIG. 9b. 1ST \& 2ND ORDER REGRESSION OF LN TRU (UPPER) ON LN GROSS ALPHA BOTH EXPERIMENTS COMBINED--NO OBSERVATIONS OMITTED

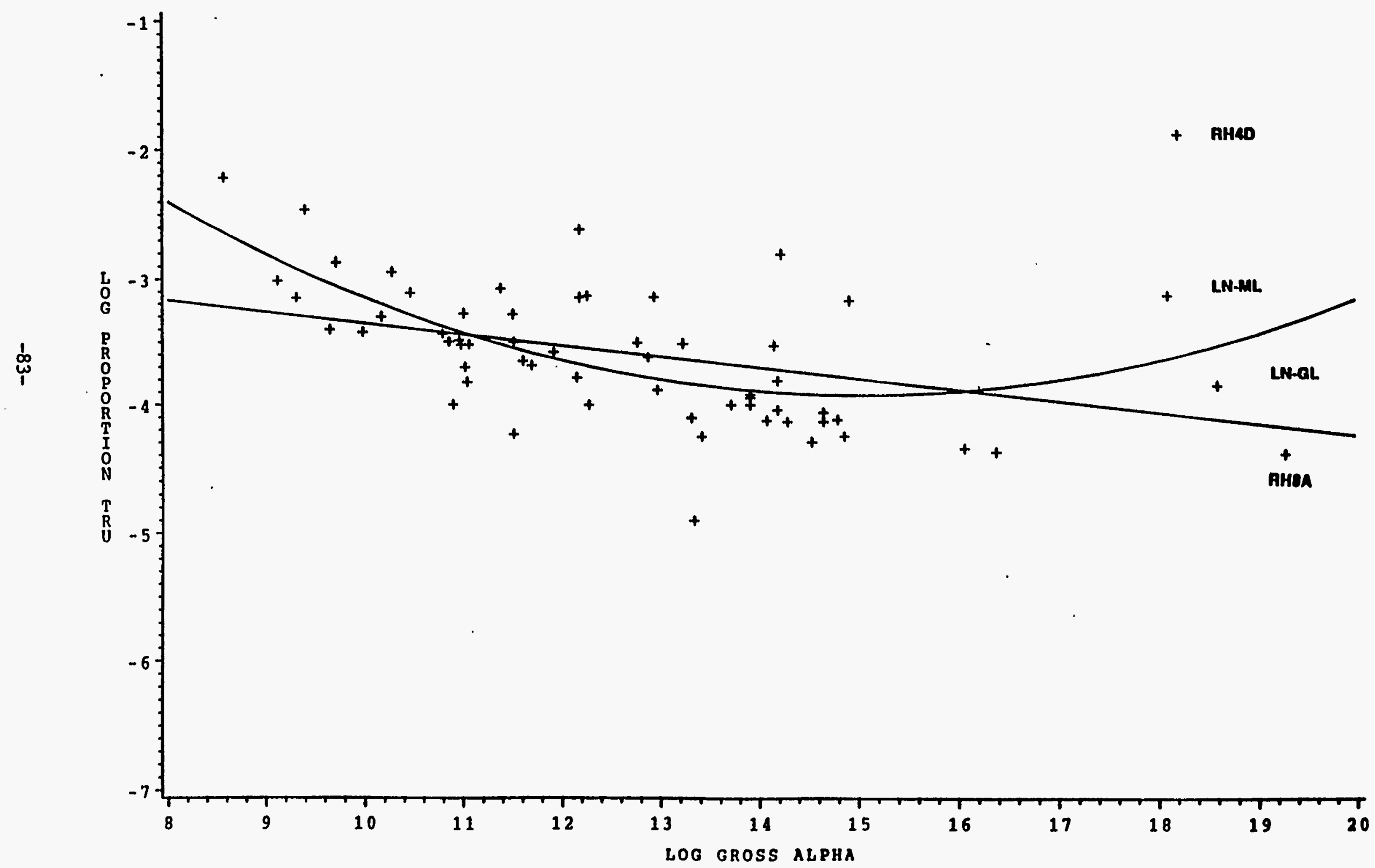


FIG. 9c. 1ST \& 2ND ORDER REGRESSION OF LN TRU (LOWER) ON LN GROSS ALPHA

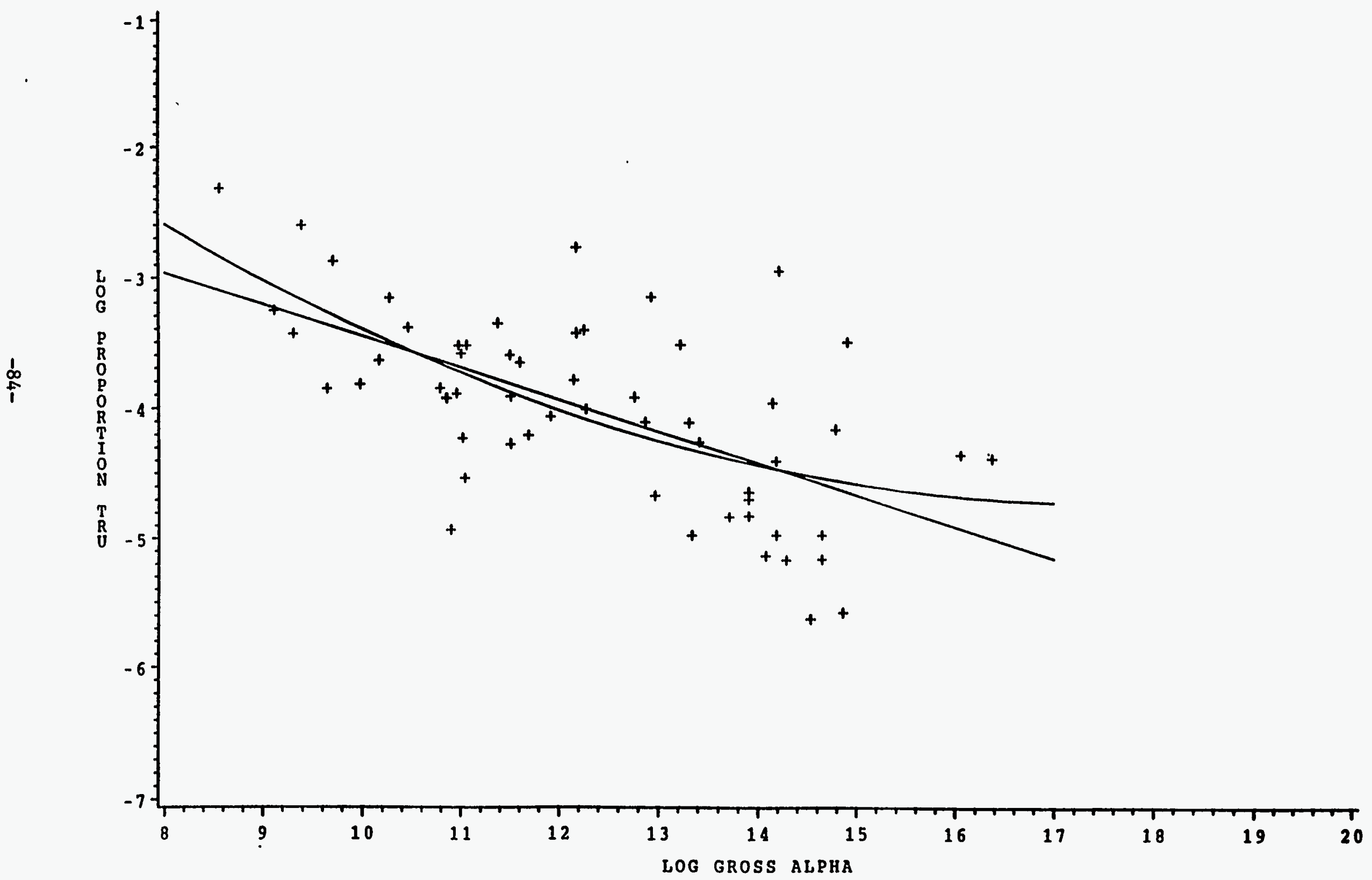


FIG. 9d. 1ST \& 2ND ORDER REGRESSION OF UN TRU (UPPER) ON LN GROSS ALPHA

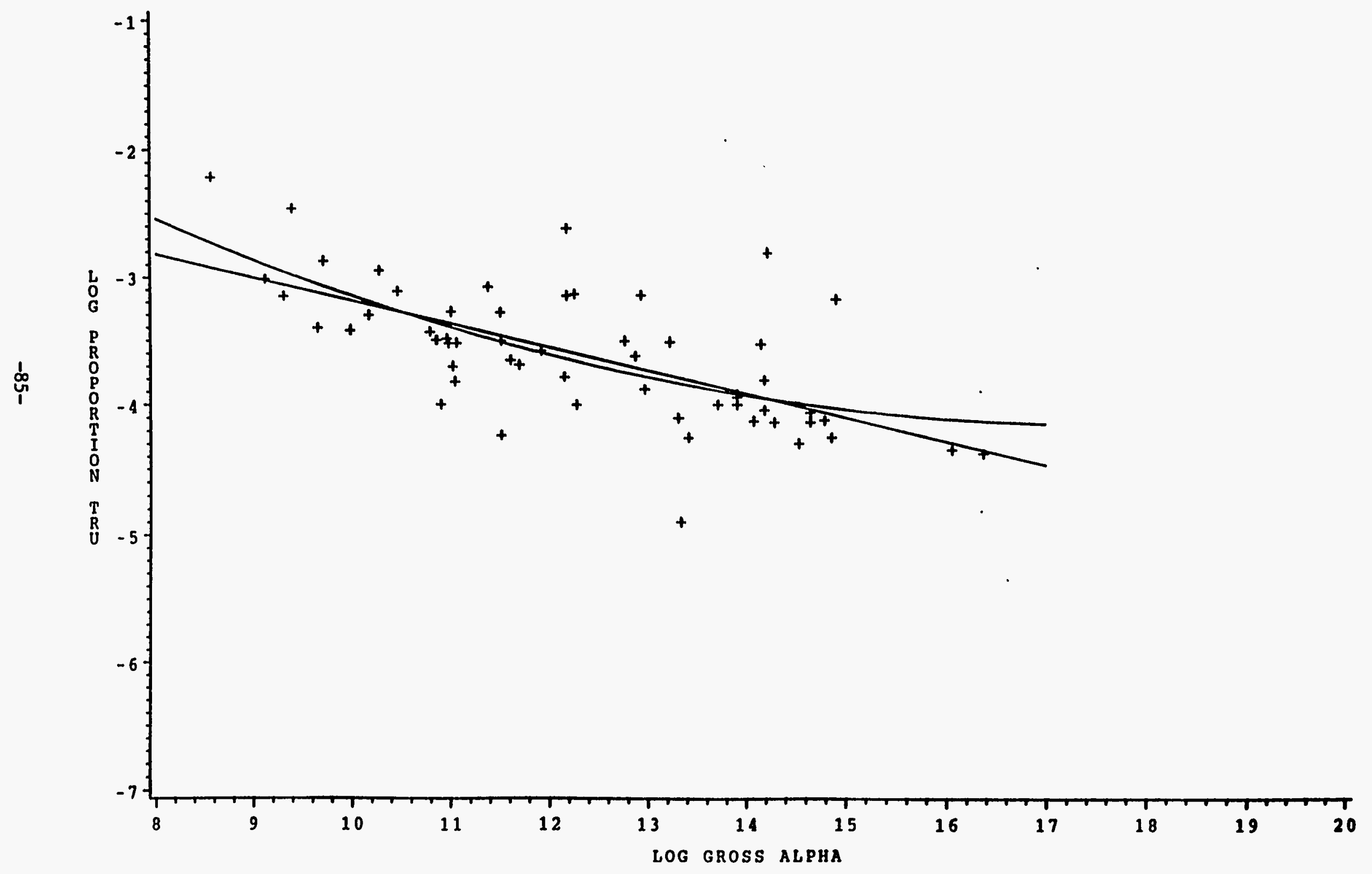


FIG. 10a. LINEAR REGRESSION OF LN TRU (LOWER) ON LN GROSS ALPHA BOTH EXPERIMENTS COMBINBD- 4 OUTLIERS OMITTED DASHED LINES BOUND 95 CONFIDENCE LIMITS ON MEAN
PU DETECTION LIMIT $=0.05 \%$ OF GROSS ALPHA

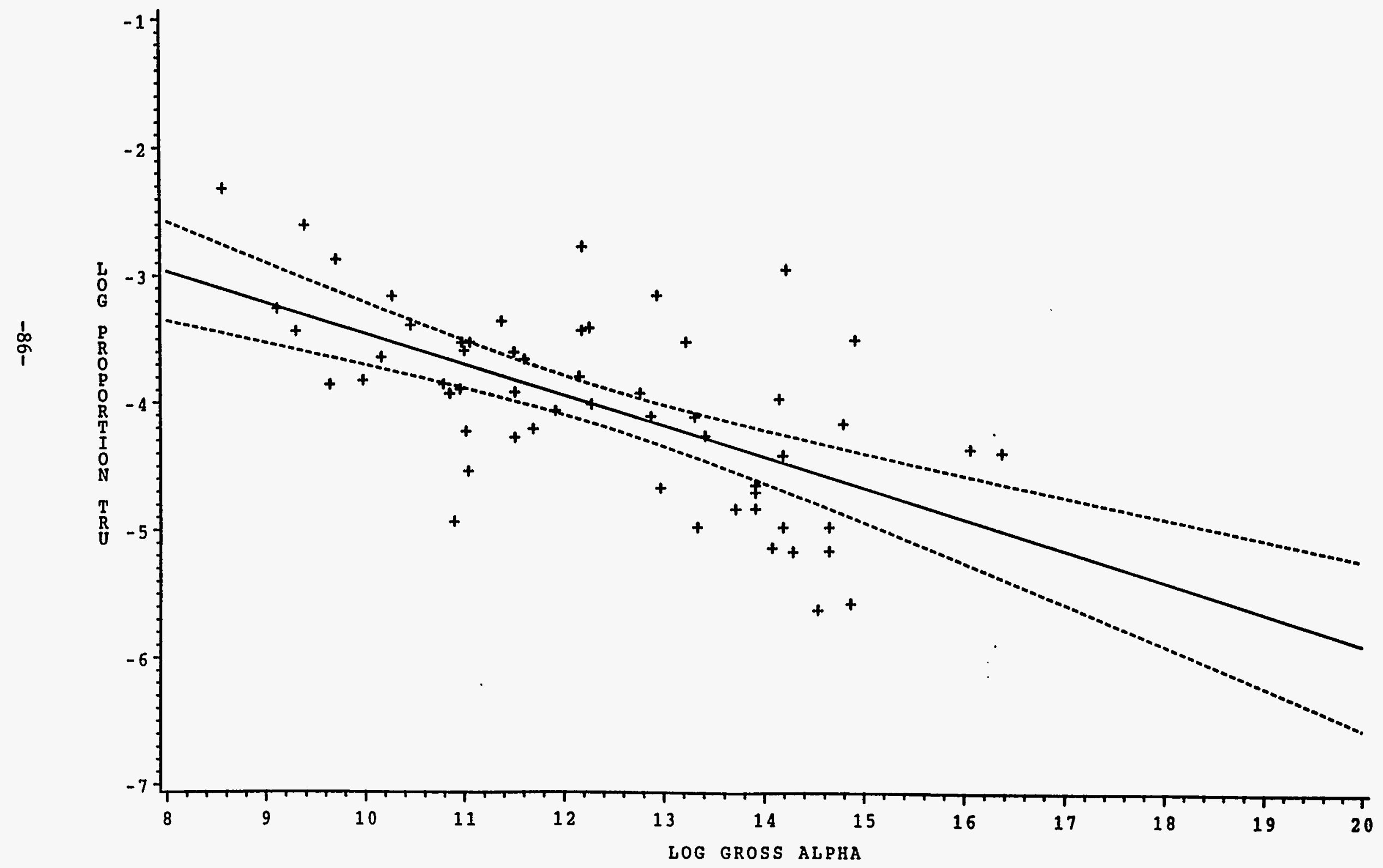


FIG. 10b. LINEAR REGRESSION OF LN TRU (UPPER) ON LN GROSS ALPHA BOTH EXPERIMENTS COMBINED--4 OUTLIERS OMITTED
DASHED LINES BOUND 958 CONFIDENCE LIMITS ON MEAN DASHED LINES BOUND 958 CONFIDENCE LIMITS ON
PU DETECTION LIMIT $=0.058$ OE GROSS ALPHA

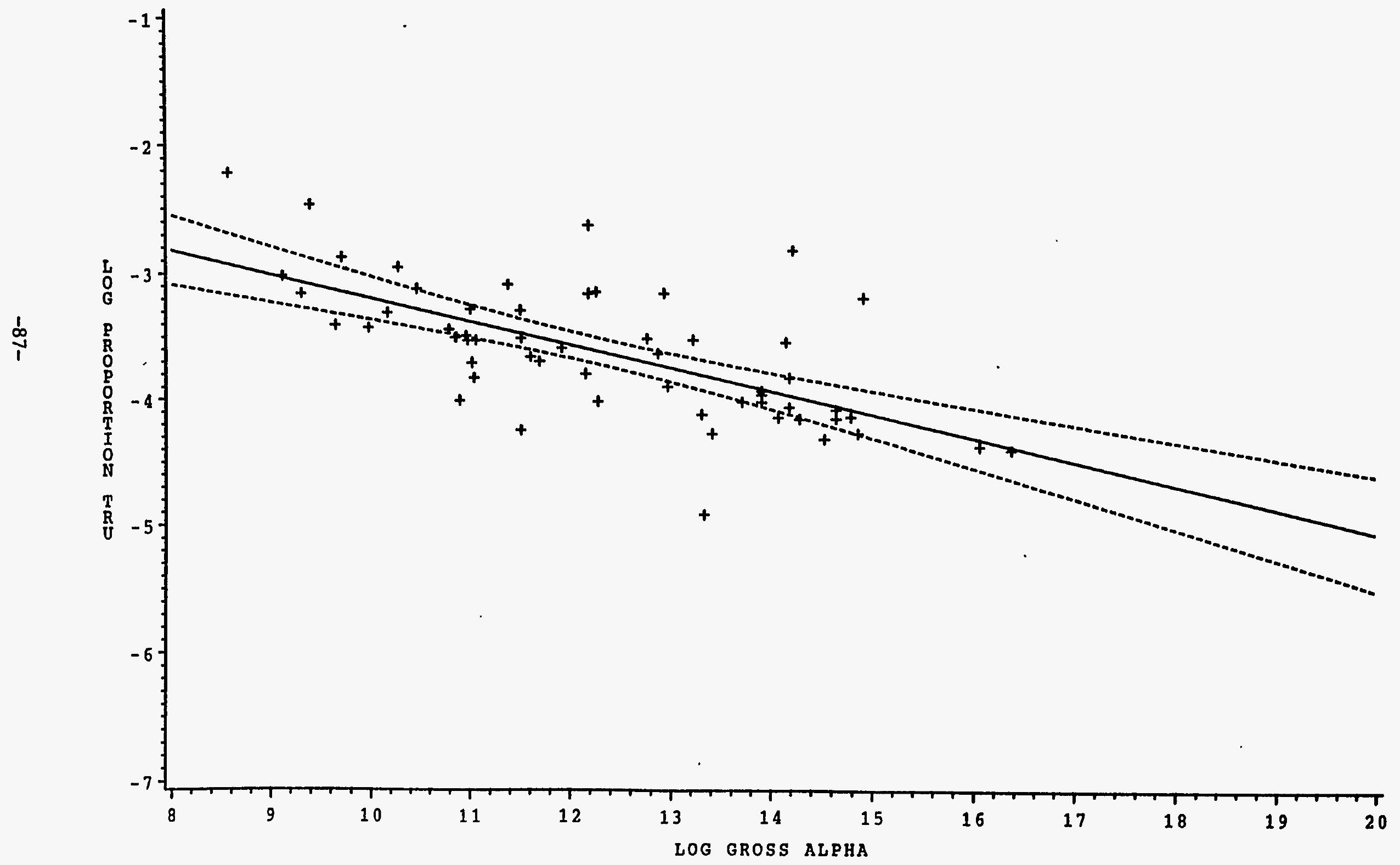




\section{FIG. 10c. LINEAR REGRESSION OF LN TRU (UPPER) ON LN GROSS ALPHA \\ BOTH EXPRRIMENTS COMBINED - -4 OUTLIERS OMITTED \\ DASHED LINES BOUND 958 CONFIDENCE LIMITS ON MEAM}

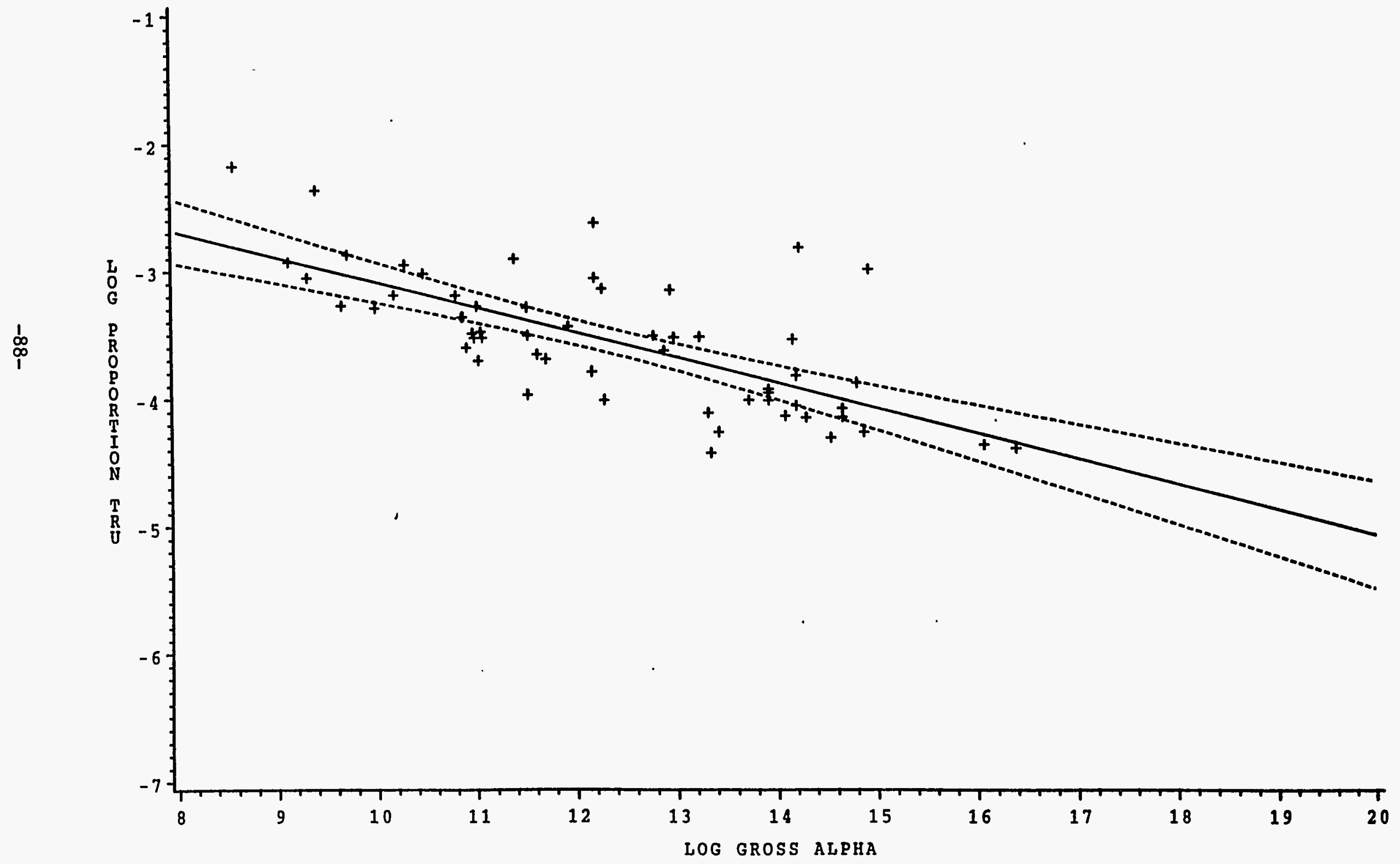


FIG. 10d. LINEAR REGRESSION OF LN TRU (UPPER) ON LN GROSS ALPHA BOTH EXPERIMENTS COMBINED-4 OUTLIERS OMITTRD

PU DETECTION LIMIT 1 . O OF GROSS ALPHA

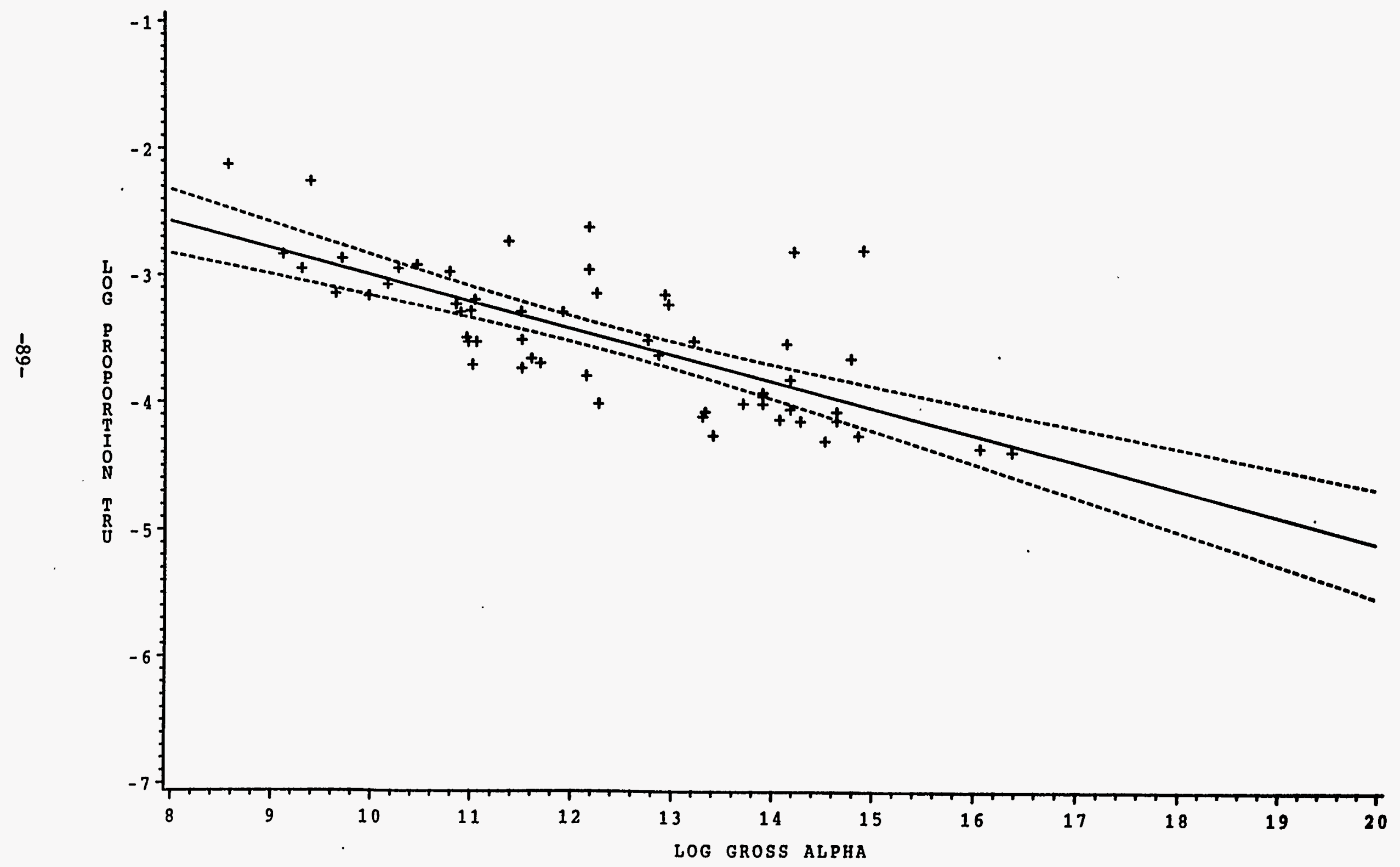




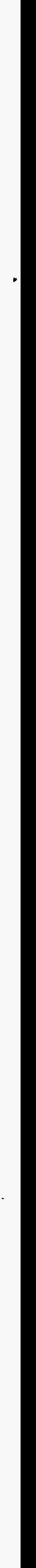




\section{INTERNAL DISTRIBUTION}

1. R. D. Bailey

2-3. J. Beauchamp

4. D. E. Benker

5. J. E. Bigelow

6. R. W. Brandenburg

7. D. E. Brashears

8-12. J. Chapman

13. D. E. Coffey

14. N. Dailey

15. T. S. Darland

16. D. Daugherty

17. J. R. DeVore

18-19. D. Downing

20. M. Evans

21-22. V. Fedorov

23. J. R. Forgy

24. W. Griest

25. J. Hackworth

26. R. Hagenauer

27. F. Homan

28. T. D. Hylton

29. J. M. Keller

30. S. Kennedy

31. T. Kent

32. F. Kornegay

33. G. R. Larson

34. M. R. Leuze

35. C. A. Manrod

36. R. Martin

37. R. C. Mason

38. B. C. McClelland
39. A. Meeks

40. G. T. Mei

41. T. Monk

42. J. Moore

43-44. L. Nguyen

45. C. E. Oliver

46-47. C. D. Parks

48. R. Peacher

49. S. Raby

50. S. Robinson

51. M. Roddye

52. T. Rogers

53. J. Saffell

54. T. F. Scanlan

55. R. E. Schreiber

56-60. F. J. Schultz

61. R. F. Sincovec

62. D. H. Smith

63. J. L. Stellern

64. J. R. Stokely

65. R. M. Wham

66. J. Williams

67. B. V. Wojtowicz

68. C. Wynn

69-70. L. Yong

71-72. Central Research Library

73. Document Reference Section

74-75. Laboratory Records Dept.

76. Laboratory Records, ORNL$\mathrm{RC}$

77-79. M\&C Records Office

\section{EXTERNAL DISTRIBUTION}

80. Eddie Ashe, Camber Corporation, 101 Donner Drive, Oak Ridge, TN 37830

81. Clark Barton, Battelle, 505 King Avenue, Columbus, OH 43201-2693

82. Greg K. Becker, Idaho National Engineering Laboratory, Lockheed Martin Idaho Technologies Company, P. O. Box 1625, MS-2114, Idaho Falls, ID 83415

83. Robert W. Benedict, Argonne National Laboratory-West, P. O. Box 2528, Idaho Falls, ID 83403 
84. Richard Bild, Sandia National Laboratories, P. O. Box 5800, MS-1335, Albuquerque, NM 87185

85. Russ Bisping, Waste Isolation Pilot Plant, U. S. Department of Energy, P. O. Box 3090, Carlsbad, NM 88221

86. Scott Boeke, U.S. Department of Energy, 3 Main, Oak Ridge, TN 37830

87. Ken Bower, Los Alamos National Laboratories, P. O. Box 1663, MS-A117, Los Alamos, NM 87545

88. Michael Brown, Waste Isolation Pilot Plant, U. S. Department of Energy, P. O. Box 3090, Carlsbad, NM 88221

89. David Camp, Lawrence Livermore National Laboratory, P. O. Box 808, MS L-333, 7000 East Ave., Livermore, CA 94550

90. Amber Clay, Waste Isolation Pilot Plant, U. S. Department of Energy, P. O. Box 3090, Carlsbad, NM 88221

91. Tom Clements, Idaho National Engineering Laboratory, Lockheed Martin Idaho Technologies Company, P. O. Box 1625, MS-2424, Idaho Falls, ID 83415

92. Don Close, Los Alamos National Laboratories, P. O. Box 1663, MS-J562, Los Alamos, NM 87545

93. Mike Connolly, Idaho National Engineering Laboratory, Lockheed Martin Idaho Technologies Company, P. O. Box 1625, MS-2424, Idaho Falls, ID 83415

94. Kenneth L. Coop, Los Alamos National Laboratories, P. O. Box 1663, MS-J562, Los Alamos, NM 87545

95. Michael E. Cournoyer, Los Alamos National Laboratories, P. O. Box 1663, MSG740, Los Alamos, NM 87545

96. Dorothy R. Davidson, Canberra Industries, Inc., 800 Research Parkway, Meriden, CT 06450

97. Pete DelMar, Los Alamos National Laboratories, P. O. Box 1663, MS-G730, Los Alamos, NM 87545

98. David S. Duncan, Argonne National Laboratory-West, P. O. Box 2528, Idaho Falls, ID 83403

99. Chris Duy, Los Alamos National Laboratories, P. O. Box 1663, MS-J593, Los Alamos, NM 87545

100. Larry East, Idaho National Engineering Laboratory, Lockheed Martin Idaho Technologies Company, P. O. Box 1625, MS-2424, Idaho Falls, ID 83415

101. Bruce R. Erdal, Los Alamos National Laboratories, P. O. Box 1663, MS-J591, Los Alamos, NM 87545

102. Deborah J. Figg, Los Alamos National Laboratories, P. O. Box 1663, MS-E518, Los Alamos, NM 87545

103. James R. Fox, Dames \& Moore, Inc., West Valley Demonstration Project, P. O. Box 191, AOC-28, West Valley, NY 14171-0191

104. Paul Frame, ORISE, P. O. Box 117, Oak Ridge, TN 37831-0117 
105. Ann Gibbs, Westinghouse Savannah River Co., P.O. Box 616, Bldg. 707-C, Aiken, SC 29802

106. Sherry Gibson, U.S. Department of Energy, 3 Main, Oak Ridge, TN 37830

107. Jeff Gross, Lockheed Martin Utility Services, P. O. Box 628, MS 2214, Piketon, OH 45669

108. Kathleen Gruetzmacher, Los Alamos National Laboratories, P. O. Box 1663, MSE501, Los Alamos, NM 87545

109. Richard Hamilton, Westinghouse Hanford Corp., P. O. Box 1970, MS-T5-06, Richland, WA 99352.

110. Richard A. Hane, Westinghouse Savannah River Co., P.O. Box 616, Bldg. 724-9E, Aiken, SC 29802

111. Yale Harker, Idaho National Engineering Laboratory, Lockheed Martin Idaho Technologies Company, P. O. Box 1625, MS-2114, Idaho Falls, ID 83415

112. Dennis L. Hjeresen, Los Alamos National Laboratories, P. O. Box 1663, MS-J591, Los Alamos, NM 87545

113. Ken Lamkin, Department of Physics, Western Kentucky University, Bowling Green, KY 42101

114. Richard Lipinski, Westinghouse Hanford Corp., P. O. Box 1970, MS-T3-04, Richland, WA 99352.

115. Charles J. Marcinkiewicz, Contech, Inc. 30 West Patrick Street, Frederick, MD 21701

116. Robert Marshall, Los Alamos National Laboratories, P. O. Box 1663, MS-G740, Los Alamos, NM 87545

117. Harry E. Martz, Jr., Lawrence Livermore National Laboratory, P. O. Box 808, MS L-333, 7000 East Ave., Livermore, CA 94550

118. Stephen Mettler, Canberra Industries, Inc., 937 W. Altgeld Street, Chicago, IL 60614

119. Stacy Butler-Moore, Department of Physics, Western Kentucky University, Bowling Green, KY 42101

120. James A. Morman, Argonne National Laboratory, 9700 S. Cass Ave., Bldg. 207, Argonne, IL 60439

121. Ed Pentaleri, SAIC, 2950 Patrick Henry Drive, Santa Clara, CA 95054

122. Elizabeth Phillips, U.S. Department of Energy, 3 Main, Oak Ridge, TN 37830

123. Mark M. Pickrell, Los Alamos National Laboratories, P. O. Box 1663, MS-E540, Los Alamos, NM 87545

124. K. K. S. Pillay, Los Alamos National Laboratories, P. O. Box 1663, MS-E500, Los Alamos, NM 87545

125. Kim Piper, Battelle Pacific National Laboratory, P. O. Box 999, Richland, WA 99352

126. M. A. Purcell, Westinghouse Hanford Corp., P. O. Box 1970, MS-T6-51, Richland, WA 99352. 
127. Gary Riner, U.S. Department of Energy, 3 Main, Oak Ridge, TN 37830

128. Kyle E. Rogers, U. S. Environmental Protection Agency, 501 3rd Street, NW, Washington, DC 20001

129. Larry Sanchez, Sandia National Laboratories, P. O. Box 5800, MS-1341, Albuquerque, NM 87185

130. Jim Sprinkle, Los Alamos National Laboratories, P. O. Box 1663, MS-E540, Los Alamos, NM 87545

131. Clifford J. Stanley, Idaho National Engineering Laboratory, Lockheed Martin Idaho Technologies Company, P. O. Box 1625, MS-4201, Idaho Falls, ID 83415

132. Lenny Storz, Sandia National Laboratories, P. O. Box 5800, MS-1341, Albuquerque, NM 87185

133. Bob Tuttle, Rocketdyne, 6633 Canoga Avenue, Canoga Park, CA 93063

134. Craig R. Tyler, Idaho National Engineering Laboratory, Lockheed Martin Idaho Technologies Company, P. O. Box 1625, MS-2424, Idaho Falls, ID 83415

135. Ruth F. Weiner, Sandia National Laboratories, P. O. Box 5800, MS-1341, Albuquerque, NM 87185

136. William W. Weston, Long Range Regulatory Compliance, Westinghouse Electric Corporation, P. O. Box 2078, MS-090, Carlsbad, NM 88221

137. Jeff Williams, U.S. Department of Energy, Trevion II, EM-36, 19901 Germantown Road, Germantown, MD 20874

138. Michael A. Zebarth, Canberra Industries, Inc., 800 Research Parkway, Meriden, CT 06450

139-140. Office of Scientific and Technical Information, P. O. Box 62, Oak Ridge, TN 37830 